\title{
Light Scalar Mesons in Photon-Photon Collisions
}

\author{
N.N. Achasov* and G.N. Shestakov ${ }^{\dagger}$ \\ Laboratory of Theoretical Physics, S.L. Sobolev Institute for Mathematics, 630090, Novosibirsk, Russia
}

\begin{abstract}
The light scalar mesons, discovered over forty years ago, became a challenge for the naive quarkantiquark model from the outset. At present the nontrivial nature of these states is no longer denied practically anybody. Two-photon physics has made a substantial contribution to understanding the nature of the light scalar mesons. Recently, it entered a new stage of high statistics measurements. We review the results concerning two-photon production mechanisms of the light scalars, based on the analysis of current experimental data.
\end{abstract}

PACS numbers: 12.39.-x, 13.40.-f, 13.60.Le, 13.75.Lb

\section{Outline}

1. Introduction.

2. Special place of the light scalar mesons in the hadron world. Evidences of their four-quark structure.

3. Light scalar mesons in the light of photon-photon collisions.

3.1. History of investigations. 3.2. Current experimental situation. 3.3. Dynamics of the reactions $\gamma \gamma \rightarrow \pi \pi$ : Born contributions and angular distributions. 3.4. Production mechanisms of scalar resonances.

4. Analysis of high statistics Belle data on the reactions $\gamma \gamma \rightarrow \pi^{+} \pi^{-}$and $\gamma \gamma \rightarrow \pi^{0} \pi^{0}$. Manifestations of the $\sigma(600)$ and $f_{0}(980)$ resonances.

5. Production of the $a_{0}(980)$ resonance in the reaction $\gamma \gamma \rightarrow$ $\pi^{0} \eta$

6. Preliminary summary.

7. Future Trends.

7.1 The $f_{0}(980)$ and $a_{0}(980)$ resonances near $\gamma \gamma \rightarrow$ $K^{+} K^{-}$and $\gamma \gamma \rightarrow K^{0} \bar{K}^{0}$ reaction thresholds. 7.2 . The $\sigma(600), f_{0}(980)$, and $a_{0}(980)$ resonances in $\gamma \gamma^{*}$ collisions. 7.3. Searches for the $J / \psi \rightarrow \omega f_{0}(980)$ and $J / \psi \rightarrow \rho a_{0}(980)$ decays. 7.4 Inelasticity of $\pi \pi$ scattering and $f_{0}(980)-a_{0}(980)$ mixing.

8. Appendix

8.1. $\gamma \gamma \rightarrow \pi \pi$. 8.2. $\gamma \gamma \rightarrow \pi^{0} \eta$. 8.3. $\gamma \gamma \rightarrow K \bar{K}$.

References.

\section{Introduction}

The scalar channels in the region up to $1 \mathrm{GeV}$ became a stumbling block of QCD because both perturbation theory and sum rules do not work in these channels. 1 At the same time the question on the nature of the light scalar mesons, $\sigma(600), \kappa(800), a_{0}(980)$, and $f_{0}(980)$

\footnotetext{
${ }^{*}$ E-mail: achasov@math.nsc.ru

${ }^{\dagger}$ E-mail: shestako@math.nsc.ru

1 The point is that, in contrast to classic vector channels, in this region there are not solitary resonances, i.e., scalar resonances, which are not accompanied by a large inseparable from resonance background. Particularly, in the case of the solitary $a_{0}(980)$ and $f_{0}(980)$ resonances, the resonance peaks in the $\phi \rightarrow \gamma a_{0}(980) \rightarrow \gamma \pi \eta$ and $\phi \rightarrow \gamma f_{0}(980) \rightarrow \gamma \pi \pi$ decays would be not observed at all because the differential probabilities of these
}

[10, 11], is major for understanding the mechanism of the chiral symmetry realization, arising from the confinement, and hence for understanding the confinement itself.

Hunting the light $\sigma$ and $\kappa$ mesons had begun in the sixties already and a preliminary information on the light scalar mesons in Particle Data Group (PDG) reviews had appeared at that time (see, for example, [12 14]). The theoretical ground for a search for scalar mesons was the linear $\sigma$ model (LSM) [15 17], which takes into account spontaneous breaking of chiral symmetry and contains pseudoscalar mesons as Goldstone bosons. The surprising thing is that after ten years it has been made clear that LSM could be the low energy realization of QCD. At the end of the sixties and at the beginning of the seventies 13, 18, 19] there were discovered the narrow light scalar resonances, the isovector $a_{0}(980)$ and isoscalar $f_{0}(980){ }^{2}$

As for the $\sigma$ and $\kappa$ mesons, long-standing unsuccessful attempts to prove their existence in a conclusive way entailed general disappointment and an information on these states disappeared from PDG reviews. One of principal reasons against the $\sigma$ and $\kappa$ mesons was the fact that the $S$ wave phase shifts, both $\pi \pi$ and $\pi K$ scattering, do not pass over $90^{\circ}$ at putative resonance masses. Nevertheless, experimental and theoretical investigation of processes, in which the $\sigma$ and $\kappa$ states could reveal themselves, had been continued.

Situation changes when we showed [6] that in LSM there is a negative background phase in the $\pi \pi$ scattering $S$ wave amplitude with isospin $I=0$, which hides the $\sigma$

decays vanish proportionally cubic function of the photon energy in a soft photon region for gauge invariance [1-[5], see Section 2. The principal role of the chiral background in the fate of the $\sigma(600)$ resonance was demonstrated in the linear $\sigma$ model [6 -9$]$. The solitary resonance approximation is nothing more than an academic exercise in the light scalar meson case.

2 In 1977 Jaffe noted that in the MIT bag model, which incorporates confinement phenomenologically, there exists the nonet of the light scalar four-quark states [20]. He suggested also that $a_{0}(980)$ and $f_{0}(980)$ might be these states with symbolic structures: $a_{0}^{+}(980)=u \bar{d} s \bar{s}, a_{0}^{0}(980)=(u s \bar{u} \bar{s}-d s \bar{d} \bar{s}) / \sqrt{2}$, $a_{0}^{-}(980)=d \bar{u} s \bar{s}$, and $f_{0}(980)=(u s \bar{u} \bar{s}+d s \bar{d} \bar{s}) / \sqrt{2}$. From that time $a_{0}(980)$ and $f_{0}(980)$ resonances came into beloved children of the light quark spectroscopy. 
meson with the result that the $\pi \pi S$ wave phase shift does not pass over $90^{\circ}$ at a putative resonance mass. It has been made clear that shielding wide lightest scalar mesons in chiral dynamics is very natural. This idea was picked up and triggered new wave of theoretical and experimental searches for the $\sigma$ and $\kappa$ mesons, see, for example, [21 28]. As a result the light $\sigma$ resonance, since 1996 , and the light $\kappa$ resonance, since 2004, appeared in the PDG reviews [29, 30].

By now there is an impressive amount of data about the light scalar mesons [10, 11, 31, 32]. The nontrivial nature of these states is no longer denied practically anybody. In particular, there exist numerous evidences in favour of their four-quark structure. These evidences are widely covered in the literature [1, 4, 31 84]. They are presented also in Sections 2-6.

One of them is the suppression of the $a_{0}(980)$ and $f_{0}(980)$ resonances in the $\gamma \gamma \rightarrow \pi^{0} \eta$ and $\gamma \gamma \rightarrow \pi \pi$ reactions, respectively, predicted in 1982 [85, 86] and confirmed by experiment [10, 11]. The elucidations of the mechanisms of the $\sigma(600), f_{0}(980)$, and $a_{0}(980)$ resonance production in the $\gamma \gamma$ collision and their quark structure are intimately related. That is why the studies of the two-photon processes are the important part of the light scalar meson physics.

It should be noted that the reactions of hadron production in photon-photon collisions are measured at $e^{+} e^{-}$colliders, i.e., the information on the transitions $\gamma \gamma \rightarrow$ hadrons is extracted from the data on the processes $e^{+} e^{-} \rightarrow e^{+} e^{-} \gamma \gamma \rightarrow e^{+} e^{-}$hadrons (Fig. 11). The most statistics is obtained by the so-called "non tag" method when hadrons only are detected and the scattered leptons are not. In this case the main contribution to the cross section of $e^{+} e^{-} \rightarrow e^{+} e^{-}$hadrons is provided by photons with very small virtualities. Therefore, this method allows to extract data on hadron production in collisions of almost real photons. The absolute majority of data on the inclusive channels $\gamma \gamma \rightarrow$ hadrons has been obtained with the use of this method. If the scattered electrons are detected (which leads to a loss of statistics), then one can investigate in addition the $Q^{2}$ dependence of the hadron production cross sections in $\gamma \gamma^{*}\left(Q^{2}\right)$ collisions, where $\gamma$ is a real photon and $\gamma^{*}\left(Q^{2}\right)$ is a photon with virtuality $Q^{2}=\left(p_{1}-p_{1}^{\prime}\right)^{2}{ }^{3}$

Recently a qualitative leap had place in the experimental investigations of the $\gamma \gamma \rightarrow \pi \pi$ and $\gamma \gamma \rightarrow \pi^{0} \eta$ processes 89 93] that proved the theoretical expectations based on the four-quark nature of the light scalar mesons [85, 86]. The Belle Collaboration published the data on the cross sections for the $\gamma \gamma \rightarrow \pi^{+} \pi^{-}$[90, 91], $\gamma \gamma \rightarrow \pi^{0} \pi^{0}$ [92], and $\gamma \gamma \rightarrow \pi^{0} \eta$ [93] reactions, statistics of which are hundreds of times as large as statistics of all previous data. The Belle Collaboration observed for the first time the clear

\footnotetext{
${ }^{3}$ Detailed formulae for experimental investigations of the reactions $e^{+} e^{-} \rightarrow e^{+} e^{-}$hadrons may be found in the reviews [87, [88].
}

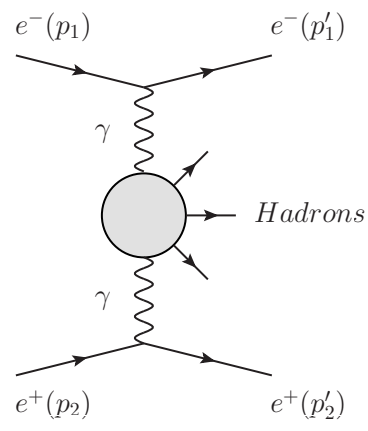

Figure 1: The two-photon process of hadron formation at $e^{+} e^{-}$ colliders; $p_{1}, p_{1}^{\prime}$ and $p_{2}, p_{2}^{\prime}$ are the 4 -momenta of electrons and positrons.

signals of the $f_{0}(980)$ resonance in the both charge channels. The previous indications for the $f_{0}(980)$ production in the $\gamma \gamma$ collisions [94-100] were rather indefinite.

In the given paper there are presented the results of the investigation the mechanisms of the $\gamma \gamma \rightarrow \pi^{+} \pi^{-}$, $\gamma \gamma \rightarrow \pi^{0} \pi^{0}$, and $\gamma \gamma \rightarrow \pi^{0} \eta$ reactions (see Sections 3-5) based on the analysis $[9,101-106]$ of the Belle data [89 93] and our previous investigations of the scalar meson physics in the $\gamma \gamma$ collisions [48, 85, 86, 107-110]. We also briefly (sometimes critical) survey analyses of other authors.

The joint analysis of the Belle high-statistics data on the $\gamma \gamma \rightarrow \pi^{+} \pi^{-}$and $\gamma \gamma \rightarrow \pi^{0} \pi^{0}$ reactions is presented and the principal dynamical mechanisms of these processes are elucidated in the energy region up to 1.5 $\mathrm{GeV}$. The analysis of the Belle high-statistics data on the reaction $\gamma \gamma \rightarrow \pi^{0} \eta$ is presented too. It is shown that the two-photon decays of the light scalar resonances are the four-quark transitions caused by the rescatterings $\sigma \rightarrow \pi^{+} \pi^{-} \rightarrow \gamma \gamma, f_{0}(980) \rightarrow\left(K^{+} K^{-}+\pi^{+} \pi^{-}\right) \rightarrow \gamma \gamma$, and $a_{0}(980) \rightarrow\left(K \bar{K}+\pi^{0} \eta+\pi^{0} \eta^{\prime}\right) \rightarrow \gamma \gamma$ in contrast to the two-photon decays of the classic $P$ wave tensor $q \bar{q}$ mesons $a_{2}(1320), f_{2}(1270)$ and $f_{2}^{\prime}(1525)$, which are caused by the direct two-quark transitions $q \bar{q} \rightarrow \gamma \gamma$ in the main. As for the direct coupling constants of the $\sigma(600), f_{0}(980)$, and $a_{0}(980)$ resonances with the $\gamma \gamma$ system, they are small. It is obtained the twophoton widths averaged over resonance mass distributions $\left\langle\Gamma_{f_{0} \rightarrow \gamma \gamma}\right\rangle_{\pi \pi} \approx 0.19 \mathrm{keV},\left\langle\Gamma_{a_{0} \rightarrow \gamma \gamma}\right\rangle_{\pi \eta} \approx 0.4 \mathrm{keV}$ and $\left\langle\Gamma_{\sigma \rightarrow \gamma \gamma}\right\rangle_{\pi \pi} \approx 0.45 \mathrm{keV}$.

In Section 7, we attend to the additional possibilities of the investigation of the $a_{0}(980)$ and $f_{0}(980)$ resonances in the reactions $\gamma \gamma \rightarrow K^{+} K^{-}$and $\gamma \gamma \rightarrow K^{0} \bar{K}^{0}$, which are as yet little studied experimentally, and also to the promising possibility of investigating the nature of the light scalars $\sigma(600), f_{0}(980)$, and $a_{0}(980)$ in $\gamma \gamma^{*}\left(Q^{2}\right)$ collisions.

\section{Special place of the light scalar mesons in the hadron world. Evidences of their four-quark structure}


Even a cursory examination of PDG reviews gives an idea of the four-quark structure of the light scalar meson nonet $^{4}, \sigma(600), \kappa(800), a_{0}(980)$, and $f_{0}(980)$,

$$
\begin{array}{cccc}
a_{0}^{-} & & a_{0}^{0} / f_{0} & a_{0}^{+} \\
& \{\kappa\} & & \{\kappa\}
\end{array}
$$

\section{$\sigma$}

inverted [49] in comparison with the classical $P$ wave $q \bar{q}$ tensor meson nonet $f_{2}(1270), a_{2}(1320), K_{2}^{*}(1420)$, and $f_{2}^{\prime}(1525)$

$$
\begin{aligned}
& f_{2}^{\prime} \\
& \left\{K_{2}^{*}\right\} \quad\left\{K_{2}^{*}\right\} \\
& a_{2}^{-} \quad a_{2}^{0} / f_{2} \quad a_{2}^{+},
\end{aligned}
$$

or also in comparison with the classical $S$ wave vector meson nonet $\rho(770), \omega(782), K^{*}(892)$, and $\phi(1020) .{ }^{5}$ In the naive quark model such a nonet cannot be understood as the $P$ wave $q \bar{q}$ nonet, but it can be easy understood as the $S$ wave $q^{2} \bar{q}^{2}$ nonet, where $\sigma(600)$ has no strange quarks, $\kappa(800)$ has the $s$ quark, $a_{0}(980)$ and $f_{0}(980)$ have the $s \bar{s}$ pair.

The scalar mesons $a_{0}(980)$ and $f_{0}(980)$, discovered about forty years ago, became the hard problem for the naive $q \bar{q}$ model from the outset. ${ }^{6}$ Really, on the one hand the almost exact degeneration of the masses of the isovector $a_{0}(980)$ and isoscalar $f_{0}(980)$ states revealed seemingly the structure $a_{0}^{+}(980)=u \bar{d}, a_{0}^{0}(980)=(u \bar{u}-d \bar{d}) / \sqrt{2}$, $a_{0}^{-}(980)=d \bar{u}$ and $f_{0}(980)=(u \bar{u}+d \bar{d}) / \sqrt{2}$ similar to the structure of the vector $\rho$ and $\omega$ or tensor $a_{2}(1320)$ and $f_{2}(1270)$ mesons, but on the other hand, the strong coupling of the $f_{0}(980)$ with the $K \bar{K}$ channel as if suggested

4 To be on the safe side, notice that the linear $\sigma$ model does not contradict to non- $q \bar{q}$ nature of the low lying scalars because Quantum Fields can contain different virtual particles in different regions of virtuality.

5 In Eqs. (1) and (2) the mass and isotopic spin third component of states increase bottom-up and from left to right, respectively.

6 Note here a series of important experiments of seventies in which the $f_{0}(980)$ and $a_{0}(980)$ resonances were investigated [11] 116], as well as a few theoretical analyses of scalar meson properties relevant to this period [20, 117-122]. In the last-named paper there was theoretically discovered the fine threshold phenomenon of the $a_{0}(980)-f_{0}(980)$ mixing which breaks the isotopic invariance (see also [123]). Now a rebirth of interest in the $a_{0}(980)-f_{0}(980)$ mixing takes place and there appear new suggestions on search for this phenomenon (see, for example, 124127] and references in these papers) as well as the first indications for its manifestation in the $f_{1}(1285) \rightarrow \pi^{+} \pi^{-} \pi^{0}$ decay, which is measured with the help of the VES detecor at IHEP in Protvino [128 [130], and in the decays $J / \psi \rightarrow \phi f_{0}(980) \rightarrow \phi a_{0}(980) \rightarrow$ $\phi \eta \pi$ and $\chi_{c 1} \rightarrow \pi^{0} a_{0}(980) \rightarrow \pi^{0} f_{0}(980) \rightarrow \pi^{+} \pi^{-} \pi^{0}$, which are being investigated with the BESIII detector at BEPCII in Chine 131.

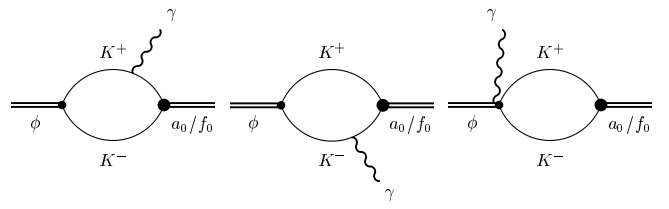

Figure 2: The $K^{+} K^{-}$loop mechanism of the radiative decays $\phi(1020) \rightarrow \gamma\left(a_{0}(980) / f_{0}(980)\right)$.

a considerable part of the strange pair $s \bar{s}$ in the wave function of the $f_{0}(980)$.

At the beginning of eighty it was demonstrated in a series of papers 34, 123, 132 136] that data on the $f_{0}(980)$ and $a_{0}(980)$ resonances, available at that time, can be interpreted in favour of the $q^{2} \bar{q}^{2}$ model, i.e., can be explained by using coupling constants of the $f_{0}(980)$ and $a_{0}(980)$ states with pseudoscalar mesons superallowed by the Okubo-Zweig-Iizuka (OZI) rule as it is predicted by the $q^{2} \bar{q}^{2}$ model. In particular, in these papers there were obtained and specified formulae for scalar resonance propagators with taking into account corrections for finite width in case of strong coupling with two-particle decay channels. Late on, these formulae were used in fitting data of a series of experiments on the $f_{0}(980)$ and $a_{0}(980)$ resonance production (see, for example, 90 92, 137-151]). Recently, it was shown that the above scalar resonance propagators satisfy the Källen-Lehmann representation in the domain of coupling constants usually used [152].

At the end of eighties it was shown that the study of the radiative decays $\phi \rightarrow \gamma a_{0} \rightarrow \gamma \pi \eta$ and $\phi \rightarrow \gamma f_{0} \rightarrow \gamma \pi \pi$ can shed light on the problem of $a_{0}(980)$ and $f_{0}(980)$ mesons [1]. Over the next ten years before experiments (1998) the question was considered from different points of view [153 165].

Now these decays have been studied not only theoretically but also experimentally with the help of the SND [137 140] and CMD-2 [141, 142] detectors at Budker Institute of Nuclear Physics in Novosibirsk and the KLOE detector at the DA $\Phi N E \phi$-factory in Frascati 143, 144, 146 148, 151, 166 168.

These experimental data called into being a series of theoretical investigations [2 5, 40, 169 172] in which evidences for the four-quark nature of the $f_{0}(980)$ and $a_{0}(980)$ states were obtained. Note the clear qualitative one. The isovector $a_{0}(980)$ resonance is produced in the radiative $\phi$ meson decay as intensively as the isoscalar $\eta^{\prime}(958)$ meson containing $\approx 66 \%$ of $s \bar{s}$, responsible for the $\phi \approx s \bar{s} \rightarrow \gamma s \bar{s} \rightarrow \gamma \eta^{\prime}(958)$ decay. In the two-quark model, $a_{0}^{0}(980)=(u \bar{u}-d \bar{d}) / \sqrt{2}$, the $\phi \approx s \bar{s} \rightarrow \gamma a_{0}(980)$ decay should be suppressed by the OZI rule. So, experiment, probably, indicates for the presence of the $s \bar{s}$ pair in the isovector $a_{0}(980)$ state, i.e., for its four-quark nature.

When basing the experimental investigations [1], it was suggested the kaon loop model $\phi \rightarrow K^{+} K^{-} \rightarrow \gamma a_{0}(980) \rightarrow \gamma \pi^{0} \eta \quad$ and $\quad \phi \rightarrow K^{+} K^{-} \rightarrow$ $\gamma f_{0}(980) \rightarrow \gamma \pi \pi$, see Fig. 2] This model is used 

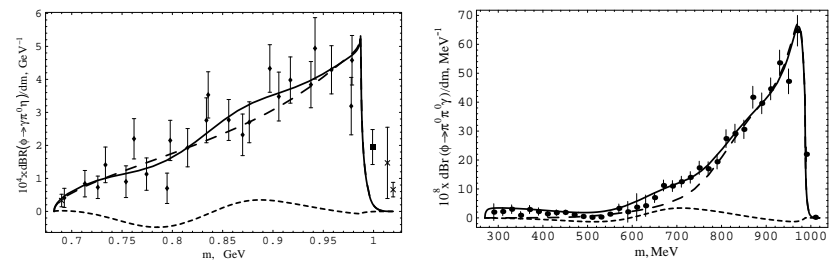

Figure 3: The left and right plots illustrate the fit to the KLOE data for the $\pi^{0} \eta$ and $\pi^{0} \pi^{0}$ mass spectra in the $\phi \rightarrow \gamma \pi^{0} \eta[143]$ and $\phi \rightarrow \gamma \pi^{0} \pi^{0}$ [144] decays, respectively. See for details [169 172]

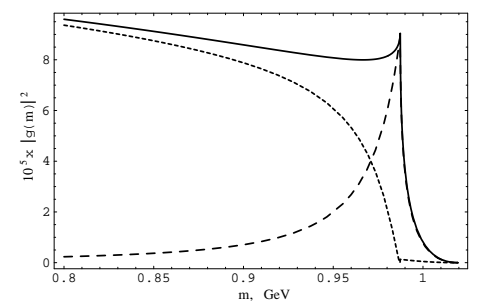

Figure 4: A new threshold phenomenon in $\phi \rightarrow K^{+} K^{-} \rightarrow \gamma R$ decays. The universal in the $K^{+} K^{-}$loop model function $|g(m)|^{2}=\left|g_{R}(m) / g_{R K^{+} K^{-}}\right|^{2}$ is drawn with the solid line. The contributions of the imaginary and real parts of $g(m)$ are drawn with the dashed and dotted lines, respectively.

in the data treatment and is ratified by experiment [137 144, 146 148, 151, 166 168, 173 175], see Fig. 3. The key virtue of the kaon loop model has the built-in nontrivial threshold phenomenon, see Fig. 4. To describe the experimental mass distributions $d B R(\phi \rightarrow \gamma R \gamma a b ; m) / d m \sim|g(m)|^{2} \omega(m),{ }^{7}$ the function $|g(m)|^{2}$ should be smooth at $m \leq 0.99 \mathrm{GeV}$. But gauge invariance requires that $g(m)$ is proportional to the photon energy $\omega(m)$. Stopping the impetuous increase of the function $(\omega(m))^{3}$ at $\omega(990 \mathrm{MeV})=29 \mathrm{MeV}$ is the crucial point in the data description. The $K^{+} K^{-}$ loop model solves this problem in the elegant way [25, 39, 40], see Fig. 4. In truth this means that $a_{0}(980)$ and $f_{0}(980)$ resonances are seen in the radiative decays of $\phi$ meson owing to the $K^{+} K^{-}$intermediate state. So the mechanism of the $a_{0}(980)$ and $f_{0}(980)$ mesons production in the $\phi$ radiative decays is established at a physical level of proof at least.

Both real and imaginary parts of the $\phi \rightarrow \gamma R$ amplitude are caused by the $K^{+} K^{-}$intermediate state. The imaginary part is caused by the real $K^{+} K^{-}$intermediate state while the real part is caused by the virtual compact $K^{+} K^{-}$intermediate state, i.e., we are dealing here with the four-quark transition [4, 5, 39, 40]. Needless to say, radiative four-quark transitions can happen between two

\footnotetext{
${ }^{7}$ Here $m$ is the invariant mass of the $a b$-state, $R=a_{0}(980)$ or $f_{0}(980), a b=\pi^{0} \eta$ or $\pi^{0} \pi^{0}$, the function $g(m)$ describes the $\phi \rightarrow$ $\gamma\left[a_{0}(m) / f_{0}(m)\right]$ transition vertex.
}

$q \bar{q}$ states as well as between $q \bar{q}$ and $q^{2} \bar{q}^{2}$ states but their intensities depend strongly on a type of the transition. A radiative four-quark transition between two $q \bar{q}$ states requires creation and annihilation of an additional $q \bar{q}$ pair, i.e., such a transition is forbidden according to the OZI rule, while a radiative four-quark transition between $q \bar{q}$ and $q^{2} \bar{q}^{2}$ states requires only creation of an additional $q \bar{q}$ pair, i.e., such a transition is allowed according to the OZI rule. The consideration of this question from the large $N_{C}$ expansion standpoint [4, 5] supports a suppression of a radiative four-quark transition between two $q \bar{q}$ states in comparison with a radiative four-quark transition between $q \bar{q}$ and $q^{2} \bar{q}^{2}$ states. So, both intensity and mechanism of the $a_{0}(980)$ and $f_{0}(980)$ production in the radiative decays of the $\phi(1020)$ meson indicate for their four-quark nature.

Note also that the absence of the decays $J / \psi \rightarrow$ $\gamma f_{0}(980), J / \psi \rightarrow a_{0}(980) \rho, J / \psi \rightarrow f_{0}(980) \omega$ against a background of the rather intensive decays into the corresponding classical $P$ wave tensor $q \bar{q}$ resonances $J / \psi \rightarrow \gamma f_{2}(1270)$ (or even $\left.J / \psi \rightarrow \gamma f_{2}^{\prime}(1525)\right), J / \psi \rightarrow$ $a_{2}(1320) \rho, J / \psi \rightarrow f_{2}(1270) \omega$ intrigues against the $P$ wave $q \bar{q}$ structure of the $a_{0}(980)$ and $f_{0}(980)$ states 36 38, 41].

\section{Light scalars in the light of two-photon collisions}

\subsection{History of investigations}

Experimental investigations of light scalar mesons in the $\gamma \gamma \rightarrow \pi^{+} \pi^{-}, \gamma \gamma \rightarrow \pi^{0} \pi^{0}$ and $\gamma \gamma \rightarrow \pi^{0} \eta$ reactions with the $e^{+} e^{-}$-colliders began in eighties and have continued up to now. In first decade many groups, DM1, DM1/2, PLUTO, TASSO, CELLO, JADE, Crystal Ball, MARK II, DELCO, and TPC $/ 2 \gamma$, took part in that. Only Crystal Ball and JADE could studied the $\pi^{0} \pi^{0}$ and $\pi^{0} \eta$ channels, the others (and JADE) the $\pi^{+} \pi^{-}$channel. For those, who wish to read more widely in the contribution of this impressive period in light scalar meson physics, one can recommend the following reviews and papers: [48, 85, 86, 88, 107, 108, 176 197].

First results on the $f_{0}(980)$ resonance production are collected in Tables \and II.

It is reasonable that first conclusions had a qualitative character and data on the $f_{0}(980) \rightarrow \gamma \gamma$ decay width had large errors or were upper bounds. Note as a guide that the TASSO and Crystal Ball results, see Table III based on the integral luminosity equals to $9.24 \mathrm{pb}^{-1}$ and $21 \mathrm{pb}^{-1}$, respectively.

As for the $a_{0}(980)$ resonance, it was observed in the $\gamma \gamma \rightarrow \pi^{0} \eta$ reaction only in three experiments. The Crystal Ball group [189] collected during two years the integral luminosity of $110 \mathrm{pb}^{-1}$, selected at that 336 events relevant to the $\gamma \gamma \rightarrow \pi^{0} \eta$ reaction in the $a_{0}(980)$ and $a_{2}(1320)$ region, see Fig. 5, and published in 1986 the following result: $\Gamma_{a_{0} \rightarrow \gamma \gamma} B\left(a_{0} \rightarrow \pi^{0} \eta\right)=\left(0.19 \pm 0.07_{-0.07}^{+0.10}\right) \mathrm{keV}$, where $\Gamma_{a_{0} \rightarrow \gamma \gamma}$ is the width of the $a_{0}(980) \rightarrow \gamma \gamma$ decay and $B\left(a_{0} \rightarrow \pi^{0} \eta\right)$ is the branching ratio of the $a_{0}(980) \rightarrow \pi^{0} \eta$ decay. The measured value of $\Gamma_{a_{0} \rightarrow \gamma \gamma} B\left(a_{0} \rightarrow \pi^{0} \eta\right)$ 
Table I: First conclusions on the $f_{0}(980)$ production in $\gamma \gamma \rightarrow \pi \pi$ (see reviews 176 -178]).

\begin{tabular}{|l|l|}
\hline Experiments & \multicolumn{1}{|c|}{ Conclusions } \\
\hline Crystal Ball & No significant $f_{0}(980)$ \\
CELLO & Hint of $f_{0}(980)$ \\
JADE & No evidence for $f_{0}(980)$ \\
TASSO & Good fit to data book values \\
& for $f_{2}(1270)$ includes $f_{0}(980)$ \\
& $(3 \sigma$ effect $)$ \\
MARK II & No significant $f_{0}(980)$ signal \\
\hline
\end{tabular}

Table II: First results on the $\gamma \gamma$ width of the $f_{0}(980)$ (see reviews [88, 176, 177, 179, 180, 184]).

\begin{tabular}{|l|l|}
\hline Experiments & \multicolumn{1}{|c|}{$\Gamma_{f_{0} \rightarrow \gamma \gamma}[\mathrm{keV}]$} \\
\hline TASSO & $(1.3 \pm 0.4 \pm 0.6) / B\left(f_{0} \rightarrow \pi^{+} \pi^{-}\right)$ \\
Crystal Ball & $<0.8 / B\left(f_{0} \rightarrow \pi \pi\right)(95 \%$ C.L.) \\
JADE & $<0.8(95 \%$ C.L.) \\
Kolanoski (1988) [184] & $0.27 \pm 0.12$ (average value) \\
\hline
\end{tabular}

characterizes the intensity of $a_{0}(980)$ production in the channel $\gamma \gamma \rightarrow a_{0}(980) \rightarrow \pi^{0} \eta$. The prehistory of this result see in Refs. 179, 185, 186. After four years, the JADE group [96] (see also [184]) obtained $\Gamma_{a_{0} \rightarrow \gamma \gamma} B\left(a_{0} \rightarrow \pi^{0} \eta\right)=(0.28 \pm 0.04 \pm 0.10) \mathrm{keV}$ based on the integral luminosity $149 \mathrm{pb}^{-1}$ and $291 \gamma \gamma \rightarrow \pi^{0} \eta$ events. The Crystal Ball [189] and JADE 96] data on the $a_{0}(980) \rightarrow \gamma \gamma$ decay have aroused keen interest, see, for example, [108, 182 184, 187, 192, 195, 196, 198, 199]. Late on, need for high-statistic data arose. But until very recently, there are no new experiments on the $\gamma \gamma \rightarrow \pi^{0} \eta$ reaction. According to the PDG reviews from 1992 to 2008, the average value for $\Gamma_{a_{0} \rightarrow \gamma \gamma} B\left(a_{0} \rightarrow \pi^{0} \eta\right)=\left(0.24_{-0.7}^{+0.8}\right) \mathrm{keV}$ [10, 196]. Only in 2009, the Belle Collaboration obtained new highstatistics data on the reaction $\gamma \gamma \rightarrow \pi^{0} \eta$ at the KEKB $e^{+} e^{-}$collider [93]. The statistics collected in the Belle experiment is 3 orders of magnitude higher than in

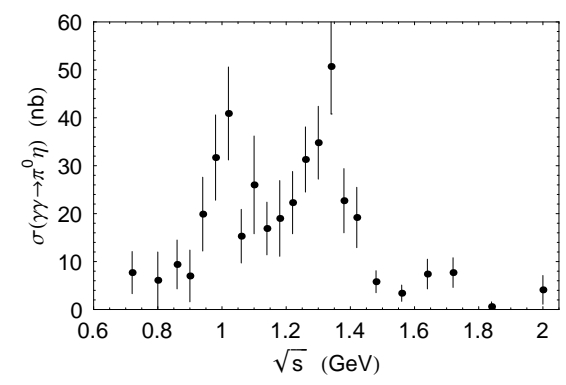

Figure 5: Cross section for $\gamma \gamma \rightarrow \pi^{0} \eta$ as a function of $\sqrt{s}$ for $|\cos \theta| \leq 0.9$, where $\sqrt{s}$ is the invariant mass of $\pi^{0} \eta$ and $\theta$ is the polar angle of the produced $\pi^{0}$ (or $\eta$ ) meson in the $\gamma \gamma$ center-ofmass system. The data are from the Crystal Ball Collaboration [189].
Table III: 1990-1992 data on the $\gamma \gamma$ width of the $f_{0}(980)$ (see the text).

\begin{tabular}{|l|l|}
\hline Experiments & \multicolumn{1}{|c|}{$\Gamma_{f_{0} \rightarrow \gamma \gamma}[\mathrm{keV}]$} \\
\hline Crystal Ball (1990) & $0.31 \pm 0.14 \pm 0.09$ \\
MARK II (1990) & $0.29 \pm 0.07 \pm 0.12$ \\
JADE (1990) & $0.42 \pm 0.06_{-0.18}^{+0.08}$ \\
Karch (1991) & $0.25 \pm 0.10$ \\
Bienlein (1992) & $0.20 \pm 0.07 \pm 0.04$ \\
& $\leq 0.31(90 \%$ CL) \\
\hline
\end{tabular}

the earlier Crystal Ball and JADE experiments. The detailed analysis of the new Belle data we present in Section 5. Here we only point out the value for $\Gamma_{a_{0} \rightarrow \gamma \gamma} B\left(a_{0} \rightarrow \pi^{0} \eta\right)=\left(0.128_{-0.002-0.043}^{+0.003+0.502}\right) \mathrm{keV}$ obtained by the authors of the experiment [93] and the average value for $\Gamma_{a_{0} \rightarrow \gamma \gamma} B\left(a_{0} \rightarrow \pi^{0} \eta\right)=\left(0.21_{-0.4}^{+0.8}\right) \mathrm{keV}$ from the last PDG review [11].

The JADE group [96] measured also the $\gamma \gamma \rightarrow \pi^{0} \pi^{0}$ cross section and having $(60 \pm 8)$-events in the $f_{0}(980)$ region (and, for comparison, $(2177 \pm 47)$ events in the $f_{2}(1270)$ region) obtained for the $f_{0} \rightarrow \gamma \gamma$ decay width $\Gamma_{f_{0} \rightarrow \gamma \gamma}=\left(0.42 \pm 0.06_{-0.18}^{+0.08}\right) \mathrm{keV}$ (that corresponds to $\Gamma_{f_{0} \rightarrow \gamma \gamma}<0.6 \mathrm{keV}$ at $95 \%$ C.L. $)$.

In addition, in 1990 the MARK II group in experiment on the $\gamma \gamma \rightarrow \pi^{+} \pi^{-}$reaction with the integral luminosity $209 \mathrm{pb}^{-1}$ [95] and the Crystal Ball group in 1990-1992 in experiments on the $\gamma \gamma \rightarrow \pi^{0} \pi^{0}$ reaction with the integral luminosities $97 \mathrm{pb}^{-1}$ [94] and $255 \mathrm{pb}^{-1}$ [98, 200] obtained also similar results for $\Gamma_{f_{0} \rightarrow \gamma \gamma}$. All data are listed together in Table III] and Figs. 6)(a) and 6(b) illustrate the manifestations of the $f_{0}(980)$ and $f_{2}(1270)$ resonances observed by MARK II and Crystal Ball in the cross sections for $\gamma \gamma \rightarrow \pi \pi$.

Although the statistical significance of the $f_{0}(980)$ signal in the cross sections and the invariant $\pi \pi$ mass resolution left much to be desired, the existence of a shoulder in the $f_{0}(980)$ resonance region in the $\gamma \gamma$ collision might be thought as established, see Fig. 6]

The experiments of eighties and beginning of nineties showed that the two-photon widths of the scalar $f_{0}(980)$ and $a_{0}(980)$ resonances are small in comparison with the two-photon widths of the tensor $f_{2}(1270)$ and $a_{2}(1320)$ resonances, for which there were obtained the following values $\Gamma_{f_{2} \rightarrow \gamma \gamma} \approx 2.6-3 \mathrm{keV}$ [94 97] (see also [10, 11]) and $\Gamma_{a_{2} \rightarrow \gamma \gamma} \approx 1 \mathrm{keV}$ [96, 189] (see also [10, 11]). This fact pointed to the four-quark nature of the $f_{0}(980)$ and $a_{0}(980)$ states 194, 96, 182 184, 187, 189, 192, 195, 196, 199, 201, 202.

As mentioned above, in the beginning of eighties it was predicted [85, 86] that, if the $a_{0}(980)$ and $f_{0}(980)$ mesons are taken as four-quark states, their production rates should be suppressed in photon-photon collisions by a factor ten in relation to the $a_{0}(980)$ and $f_{0}(980)$ mesons taken as two-quark $P$ wave states. The estimates 

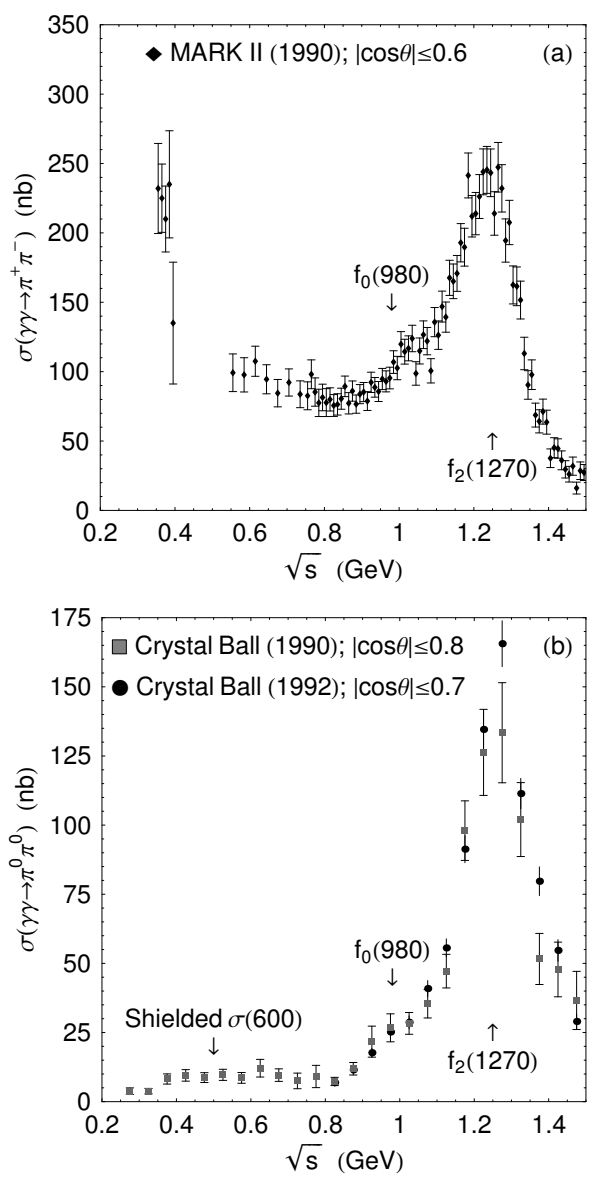

Figure 6: Cross sections for $\gamma \gamma \rightarrow \pi^{+} \pi^{-}$(a) and $\gamma \gamma \rightarrow \pi^{0} \pi^{0}$ (b) as functions of the invariant mass $\sqrt{s}$ of $\pi \pi$. The data correspond to limited angular ranges of the registration of the final pions; $\theta$ is the polar angle of the produced $\pi$ meson in the $\gamma \gamma$ center-of-mass system.

obtained for the four-quark model were 85,86$]$

$$
\Gamma_{a_{0} \rightarrow \gamma \gamma} \sim \Gamma_{f_{0} \rightarrow \gamma \gamma} \sim 0.27 \mathrm{keV}
$$

which were supported by experiments. As for the $q \bar{q}$ model, it predicted that

$$
\frac{\Gamma_{0^{++} \rightarrow \gamma \gamma}}{\Gamma_{2^{++} \rightarrow \gamma \gamma}}=\frac{15}{4} \times \text { corrections } \approx 1.3-5.5
$$

for the $P$ wave states with $J^{P C}=0^{++}$and $2^{++}$from the same family, see, for example, 36, 94, 108, 187, 192, 195, 198, 199, 203 211. The factor $\frac{15}{4}$ is obtained in the non-relativistic quark model according to which $\Gamma_{0^{++} \rightarrow \gamma \gamma}=(256 / 3) \alpha^{2}\left|R^{\prime}(0)\right|^{2} / M^{4}$ and $\Gamma_{2^{++} \rightarrow \gamma \gamma}=$ $(1024 / 45) \alpha^{2}\left|R^{\prime}(0)\right|^{2} / M^{4}$, where $R^{\prime}(0)$ is the derivative of the $P$ state radial wave function with a mass $M$ at the origin. $\Gamma_{2^{++} \rightarrow \gamma \gamma}$ differs from $\Gamma_{0^{++} \rightarrow \gamma \gamma}$ by the product of the Clebsch-Gordan spin-orbit coefficient squared $\left(\frac{1}{2}\right)$ and value of $\sin ^{4} \vartheta$ averaged over the solid angle $\left(\frac{8}{15}\right)$; see Ref. [205] for details. This suggested that $\Gamma_{f_{0} \rightarrow \gamma \gamma} \geq 3.4 \mathrm{keV}$ and $\Gamma_{a_{0} \rightarrow \gamma \gamma} \geq 1.3 \mathrm{keV}$.
One dwells else on predictions of the molecule model in which the $a_{0}(980)$ and $f_{0}(980)$ resonances are non-relativistic bound states of the $K \bar{K}$ system 212, 213]. As the $q^{2} \bar{q}^{2}$ model, the molecule one explains the state mass degeneracy and their strong coupling with the $K \bar{K}$ channel. As in the four-quark model, in the molecular one no questions arise with the small rates $B\left[J / \psi \rightarrow a_{0}(980) \rho\right] / B\left[J / \psi \rightarrow a_{2}(1320) \rho\right]$ and $B\left[J / \psi \rightarrow f_{0}(980) \omega\right] / B\left[J / \psi \rightarrow f_{2}(1270) \omega\right]$ (see specialities in Refs. [36, 38]). However, the predictions of this model for the two-photon widths [187, 198],

$$
\Gamma_{a_{0}(K \bar{K}) \rightarrow \gamma \gamma}=\Gamma_{f_{0}(K \bar{K}) \rightarrow \gamma \gamma} \approx 0.6 \mathrm{keV}
$$

are rather big, within two standard deviations contradict the experiment data from Table III. More than that, the widths of $K \bar{K}$ molecules must be smaller (strictly speaking, much smaller) than the binging energy $\epsilon \approx 10 \mathrm{MeV}$. Recent data 11], however, contradict this, $\Gamma_{a_{0}} \sim(50-100) \mathrm{MeV}$ and $\Gamma_{f_{0}} \sim(40-100) \mathrm{MeV}$. The $K \bar{K}$ molecule model predicted also 157, 162 that $B\left[\phi \rightarrow \gamma a_{0}(980)\right] \approx B\left[\phi \rightarrow \gamma f_{0}(980)\right] \sim 10^{-5}$ that contradicts experiment [11]. In addition, recently [214, 215] it was shown that the kaon loop model, ratified by experiment, describes production of a compact state and not an extended molecule. Finally, experiments in which the $a_{0}(980)$ and $f_{0}(980)$ mesons were produced in the $\pi^{-} p \rightarrow \pi^{0} \eta n$ 216, 217] and $\pi^{-} p \rightarrow \pi^{0} \pi^{0} n$ 218 220] reactions within a broad range of four-momentum transfer squared, $0<-t<1 \mathrm{GeV}^{2}$, have shown that these states are compact, e.g. as two-quark $\rho, \omega, a_{2}(1320), f_{2}(1270)$ and other mesons and not as extended molecule ones with form factors determined by the wave functions. These experiments have left no chances for the $K \bar{K}$ molecule model. ${ }^{8}$ As to four-quark states, they are as compact as two-quark states. ${ }^{9}$

The Particle Data Group gives information on an average value of $\Gamma_{f_{0} \rightarrow \gamma \gamma}$ beginning from 1992. Note that no new experimental data on $\Gamma_{f_{0} \rightarrow \gamma \gamma}$ emerged from 1992 up to 2006, nevertheless, its average value, adduced by PDG, evolved noticeably in this period. Based on the data in Table III, the $\Gamma_{f_{n} \rightarrow \gamma \gamma}$ value would be $(0.26 \pm 0.08) \mathrm{keV}$. In $1992 \mathrm{PDG}$ [196] obtained the average value $\Gamma_{f_{0} \rightarrow \gamma \gamma}=(0.56 \pm 0.11) \mathrm{keV}$ combining the JADE result (1990) [96], see Table [II] with the value $\Gamma_{f_{0} \rightarrow \gamma \gamma}=(0.63 \pm 0.14) \mathrm{keV}$, which was found by Morgan and Pennington (1990) 208 as a result of a theoretical analysis of the MARK II (1990) [95] and Crystal Ball (1990) 94] data. In 1999 Boglione and Pennington

\footnotetext{
8 A $K \bar{K}$ formation of unknown origin with the average relativistic Euclidean momentum squared $\left\langle k^{2}\right\rangle \approx 2 \mathrm{GeV}^{2}$ was considered recently and named "a $K \bar{K}$ molecule" 221]. Such a free use of the molecule term can mislead readers considering a molecule as an extent non-relativistic bound system.

9 An additional argument against the molecular model for the $a_{0}(980)$ resonance is presented in Section 5.
} 

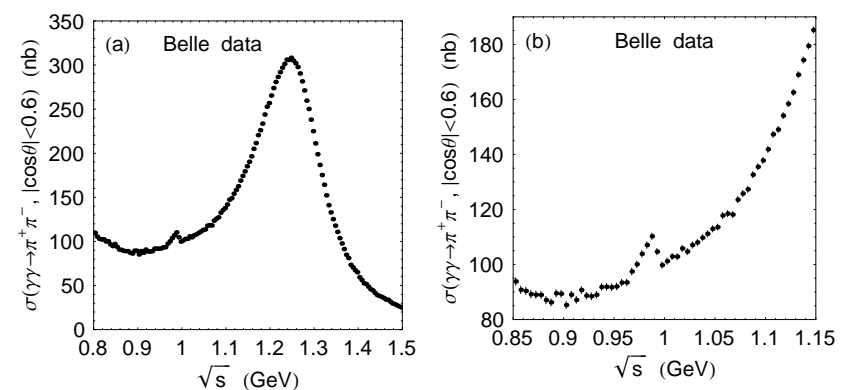

Figure 7: (a) The high statistics Belle data on the $\gamma \gamma \rightarrow \pi^{+} \pi^{-}$ reaction cross section for $|\cos \theta| \leq 0.6$ [91]. Plot (b) emphasizes the region of the $f_{0}(980)$ peak. Errors shown include statistics only. They are approximately equal to $0.5 \%-1.5 \%$. The $\sqrt{s}$ bin size in the Belle experiment has been chosen to be $5 \mathrm{MeV}$, with the mass resolution of about $2 \mathrm{MeV}$.

carried out a new theoretical analysis [222] of the situation and halved value, $\Gamma_{f_{0} \rightarrow \gamma \gamma}=\left(0.28_{-0.13}^{+0.09}\right) \mathrm{keV}$ (see also [223]). The Particle Data Group noted that the Boglione and Pennington (1999) result replaces the Morgan and Pennington (1990) one but used both results coupled with the JADE (1990) one for calculation of the average $f_{0} \rightarrow \gamma \gamma$ decay width. In this way the value $\Gamma_{f_{0} \rightarrow \gamma \gamma}=\left(0.39_{-0.13}^{+0.10}\right) \mathrm{keV}$ emerged in the PDG review (2000) 224].

In 2003 preliminary super-statistics Belle data on $\gamma \gamma \rightarrow \pi^{+} \pi^{-}$were reported. They contain a clear signal from the $f_{0}(980)$ resonance [89]. In 2005 there emerged our first response [101] to these data. It has become clear that $\Gamma_{f_{0} \rightarrow \gamma \gamma}$ is bound to be small. In 2006 PDG excluded the Morgan and Pennington (1990) result, $\Gamma_{f_{0} \rightarrow \gamma \gamma}=(0.63 \pm 0.14) \mathrm{keV}$, from its sample and using only the JADE (1990) data and the Boglione and Pennington (1999) result obtained a new guide $\Gamma_{f_{0} \rightarrow \gamma \gamma}=\left(0.31_{-0.11}^{+0.08}\right) \mathrm{keV}[225]$. To the effect that happened later to the average value of $\Gamma_{f_{0} \rightarrow \gamma \gamma}$ and can else happens to the one, we are going to tell in the following subsections 3.2 and 3.4 .

\subsection{Current experimental situation}

In 2007 the Belle collaboration published the data on cross section of the $\gamma \gamma \rightarrow \pi^{+} \pi^{-}$reaction in the region of the $\pi^{+} \pi^{-}$invariant mass, $\sqrt{s}$, from 0.8 up $1.5 \mathrm{GeV}$ based on the integral luminosity $85.9 \mathrm{fb}^{-1}[90,91]$. These data are shown on Fig. 17. Thanks to the huge statistics and high energy resolution in the Belle experiment, the clear signal of the $f_{0}(980)$ resonance was detected for the first time. Its value proved to be small that agrees qualitatively with the four-quark model prediction 85, 86]. The visible height of the $f_{0}(980)$ peak amounts of about $15 \mathrm{nb}$ over the smooth background near $100 \mathrm{nb}$. Its visible (effective) width proved to be about $30-35 \mathrm{MeV}$, see Fig. 7 .

Then the Belle collaboration published the data on cross section for the $\gamma \gamma \rightarrow \pi^{0} \pi^{0}$ reaction in the region of the $\pi^{+} \pi^{-}$invariant mass, $\sqrt{s}$, from 0.6 to $1.6 \mathrm{GeV}$
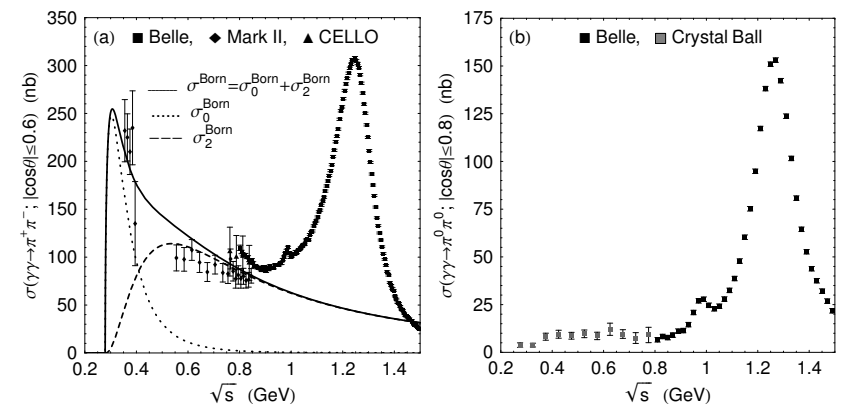

Figure 8: (a) The data on the $\gamma \gamma \rightarrow \pi^{+} \pi^{-}$reaction cross section from Mark II 94] and CELLO 97], for $\sqrt{s} \leq 0.85 \mathrm{GeV}$, and from Belle 91], for $0.8 \leq \sqrt{s} \leq 1.5 \mathrm{GeV}$. (b) The data on the $\gamma \gamma \rightarrow \pi^{0} \pi^{0}$ reaction cross section from Crystal Ball [95], for $\sqrt{s}<0.8 \mathrm{GeV}$, and from Belle [92], for $0.8 \leq \sqrt{s} \leq 1.5 \mathrm{GeV}$. Plots (a), for $\sqrt{s}>0.85 \mathrm{GeV}$, and (b), for $\sqrt{s}>0.8 \mathrm{GeV}$, show exclusively the Belle data to emphasize the discovered miniature signals from the $f_{0}(980)$ resonance. The theoretical curves, shown on plot (a), correspond to the cross sections for the process $\gamma \gamma \rightarrow \pi^{+} \pi^{-}$ for $|\cos \theta| \leq 0.6$ caused by the electromagnetic Born contribution from the elementary one pion exchange: the total integrated cross section $\sigma^{\text {Born }}=\sigma_{0}^{\text {Born }}+\sigma_{2}^{\text {Born }}$ and the integrated cross sections $\sigma_{\lambda}^{\text {Born }}$ with helicity $\lambda=0$ and 2 .

Table IV: The current data on the $f_{0}(980) \rightarrow \gamma \gamma$ decay width.

\begin{tabular}{|l|l|}
\hline Experiments & \multicolumn{1}{|c|}{$\Gamma_{f_{0} \rightarrow \gamma \gamma}[\mathrm{keV}]$} \\
\hline$\gamma \gamma \rightarrow \pi^{+} \pi^{-}$Belle (2007) [90] & $0.205_{-0.083-0.117}^{+0.095+0.147}$ \\
$\gamma \gamma \rightarrow \pi^{0} \pi^{0}$ Belle $(2008)[\underline{[92]}$ & $0.286 \pm 0.017_{-0.070}^{+0.211}$ \\
\hline PDG average value [10, 11] & $0.29_{-0.06}^{+0.07}$ \\
\hline
\end{tabular}

based on the integral luminosity $95 \mathrm{fb}^{-1}$ [92]; see also 226 228]. Here also the clear signal of the $f_{0}(980)$ resonance was detected for the first time. Note that the background conditions for the manifestation of the $f_{0}(980)$ in the $\gamma \gamma \rightarrow \pi^{0} \pi^{0}$ channel are more favourable than in the $\gamma \gamma \rightarrow \pi^{+} \pi^{-}$one.

Figures $8(a)$ and $8(b)$ illustrate a general picture of data on the cross sections of the $\pi^{+} \pi^{-}$and $\pi^{0} \pi^{0}$ production in photon-photon collisions from the $\pi \pi$ threshold up to $1.5 \mathrm{GeV}$ after the Belle experiments. It is instructive to compare these results with a previous picture illustrated by Figs. 6(a) and 6(b).

The current information about $\Gamma_{f_{0} \rightarrow \gamma \gamma}$ are adduced in Table IV] The Belle collaboration determined $\Gamma_{f_{0} \rightarrow \gamma \gamma}$ (see Table IV] as a result of fitting the mass distributions (see Figs. 7(b) and $8(\mathrm{~b})$ ) taking into account the $f_{0}(980)$ and $f_{2}(1270)$ resonance contributions and smooth background contributions, which are a source of large systematic errors in $\Gamma_{f_{0} \rightarrow \gamma \gamma}$ (see for details in Refs. 90 92]).

\subsection{Dynamics of the reactions $\gamma \gamma \rightarrow \pi \pi$ : Born contributions and angular distributions}

To feel the values of the cross sections measured by experiment, in Fig. 8 (a) the total Born cross section of the $\gamma \gamma \rightarrow \pi^{+} \pi^{-}$process, $\sigma^{\text {Born }}=\sigma_{0}^{\text {Born }}+\sigma_{2}^{\text {Born }}$, and the par- 


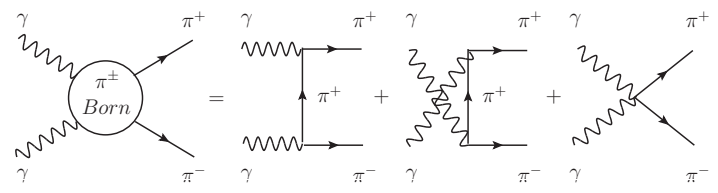

Figure 9: The Born diagrams for $\gamma \gamma \rightarrow \pi^{+} \pi^{-}$.
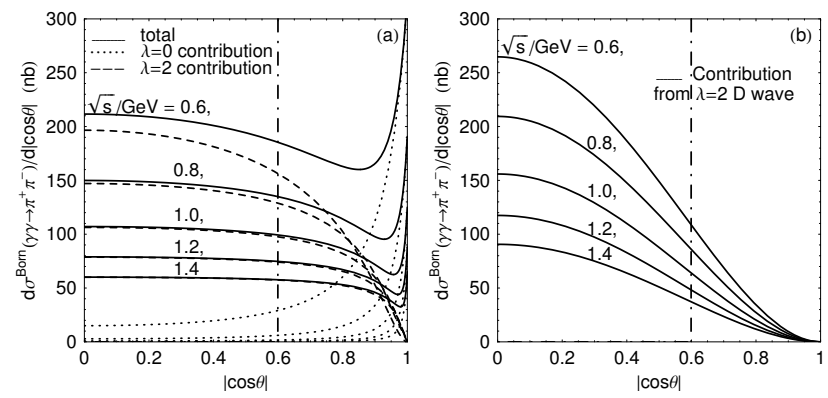

Figure 10: Plots (a) and (b) show the $\gamma \gamma \rightarrow \pi^{+} \pi^{-}$differential cross section in the Born approximation (i.e., for the elementary one pion exchange mechanism) and its components for different values of $\sqrt{s} / \mathrm{GeV}$. The vertical straight lines $|\cos \theta|=0.6$ show the upper boundary of the region available for measurements.

tial helicity ones, $\sigma_{\lambda}^{\text {Born }}$, are adduced as a guide, where $\lambda=0$ or 2 is the absolute value of the photon helicity difference. These cross sections are caused by the elementary one pion exchange mechanism, see Fig. 9, By the Low theorem ${ }^{10}$ and chiral symmetry ${ }^{11}$, the Born contributions should dominate near the threshold region of the $\gamma \gamma \rightarrow \pi^{+} \pi^{-}$reaction. As shown in Fig. 8 (a), this anticipation does not contradict the current data near threshold, but, certainly, errors leave much to be desired. In additional, one can consider the Born contributions as an reasonable approximation of background (nonresonance) contributions in the $\gamma \gamma \rightarrow \pi^{+} \pi^{-}$amplitudes in all the resonance region, including the $f_{2}(1270)$ one. The Born contributions are also the base for a construction of amplitudes, including strong interactions in final state, see, for example, 9, 101, 181, 190, 193, 194, 232239].

The Born contributions have the following particular qualities. First, $\sigma^{\text {Born }}$ has a maximum at $\sqrt{s} \approx 0.3 \mathrm{GeV}$, where $\sigma^{\text {Born }} \approx \sigma_{0}^{\text {Born }}$, then $\sigma_{0}^{\text {Born }}$ falls with increasing $\sqrt{s}$, so that the $\sigma_{2}^{\text {Born }}$ contribution dominates in $\sigma^{\text {Born }}$ at $\sqrt{s}>0.5 \mathrm{GeV}$, see Fig. 8(a). Second, although the $\sigma_{2}^{\text {Born }}$ value is approximately $80 \%$ caused by the $D$ wave amplitude, its interference with the contribution of higher waves are considerable in the differential cross section

10 According to this theorem [229 231] , the Born contributions give the exact physical amplitude of the crossing reaction $\gamma \pi^{ \pm} \rightarrow \gamma \pi^{ \pm}$ close to its threshold.

11 Chiral symmetry guarantees weakness of the $\pi \pi$ interaction at low energy. $d \sigma^{\text {Born }}\left(\gamma \gamma \rightarrow \pi^{+} \pi^{-}\right) / d|\cos \theta|$, compare Figs. 10(a) and 10(b). The interference, destructive in the first half of the $|\cos \theta| \leq 0.6$ interval and constructive in the second one, flattens out the $\theta$ angle distribution in this interval, so that this effect increases with increasing $\sqrt{s}$, see Fig. 10(a).

Since the first resonance with $I^{G}\left(J^{P C}\right)=0^{+}\left(4^{++}\right)$ has the mass near $2 \mathrm{GeV}[10,11]$, then seemingly the $S$ and $D$ wave contributions only should dominate at $\sqrt{s} \leq 1.5 \mathrm{GeV}$ and the differential cross section of the $\gamma \gamma \rightarrow \pi^{+} \pi^{-}$process could be represented as [91]

$$
d \sigma\left(\gamma \gamma \rightarrow \pi^{+} \pi^{-}\right) / d \Omega=\left|S+D_{0} Y_{2}^{0}\right|^{2}+\left|D_{2} Y_{2}^{2}\right|^{2},
$$

where $S, D_{0}$, and $D_{2}$ are the $S$ and $D_{\lambda}$ wave amplitudes with the helicity $\lambda=0$ and $2, Y_{J}^{m}$ are the spherical harmonics. ${ }^{12}$ But, the above discussion shows that the smooth background contribution in the $\gamma \gamma \rightarrow \pi^{+} \pi^{-}$cross section contains the high partial wave due to the one pion exchange, so that the smooth background can imitate the large $S$ wave at $|\cos \theta| \leq 0.6$.

The one-pion exchange is absent in the $\gamma \gamma \rightarrow \pi^{0} \pi^{0}$ channel and the representation of the cross section of this reaction similar to Eq. (6) is a good approximation at $\sqrt{s} \leq 1.5 \mathrm{GeV}$

$$
d \sigma\left(\gamma \gamma \rightarrow \pi^{0} \pi^{0}\right) / d \Omega=\left|\widetilde{S}+\widetilde{D}_{0} Y_{2}^{0}\right|^{2}+\left|\widetilde{D}_{2} Y_{2}^{2}\right|^{2},
$$

where $\widetilde{S}, \widetilde{D}_{0}$, and $\widetilde{D}_{2}$ are the $S$ and $D_{\lambda}$ wave amplitudes with the helicity $\lambda=0$ and 2 . Nevertheless, the partial wave analysis of the $\gamma \gamma \rightarrow \pi^{0} \pi^{0}$ events, based on Eq. (7), is not prevented from difficulties for the relation $\sqrt{6}\left|Y_{2}^{2}\right|=\sqrt{5} Y_{0}^{0}-Y_{2}^{0}$, which gives no way of separating the partial waves when using only the data on the differential cross section [91, 92, 96]. So, the separation of the contributions with the different helicities requests some guesswork, for example, the domination of the helicity 2 in the $f_{2}(1270)$ resonance production [209, 240 242] that agrees rather well with the experimental angle distribution.

The $d \sigma\left(\gamma \gamma \rightarrow \pi^{0} \pi^{0}\right) / d \Omega$ differential cross section in Eq. (77) is a polynomial of the second power of $z=\cos ^{2} \theta$, which can be expressed in terms of its roots $z_{1}$ and $z_{1}^{*},{ }^{13}$

$$
d \sigma\left(\gamma \gamma \rightarrow \pi^{0} \pi^{0}\right) / d \Omega=C\left(z-z_{1}\right)\left(z-z_{1}^{*}\right),
$$

where $C$ is a real quantity. So, from fitting experimental data on the differential cross section one can determine only three independent parameters, for example, $C, \operatorname{Re} z_{1}$, and $\operatorname{Im} z_{1}$ up to the sign and not four ones, $|\widetilde{S}|$,

12 Eq. (6) corresponds the situation "untagget" when the dependence on the pion azimuth $\varphi$ is not measured, that took place in all above experiments.

13 Such a procedure is the base of the determination all solutions when carrying out partial wave analyses, see, for example, 120, 217, 219, 220, 243 245]. 

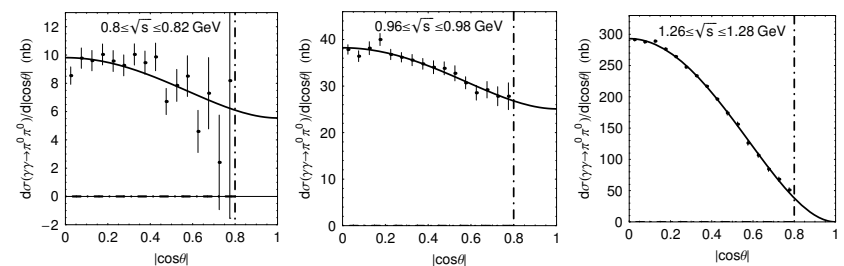

Figure 11: The Belle data on the angular distributions for $\gamma \gamma \rightarrow \pi^{0} \pi^{0}$ [92]. The solid lines are the approximations. The vertical straight lines $|\cos \theta|=0.8$ show the upper boundary of the region available for measurements.

$\left|\widetilde{D}_{0}\right|,\left|\widetilde{D}_{2}\right|$, and $\cos \delta$ ( $\delta$ is a relative phase between the $\widetilde{S}$ and $\widetilde{D}_{0}$ amplitudes), as one would like.

In Fig. 11 the Belle data on the angular distributions in $\gamma \gamma \rightarrow \pi^{0} \pi^{0}$ are adduced at three values of $\sqrt{s}$. All of them are described very well by the simple twoparameter expression $|a|^{2}+\left|b Y_{2}^{2}\right|^{2}$ [103]. This suggests that the $\gamma \gamma \rightarrow \pi^{0} \pi^{0}$ cross section is saturated only by the $\widetilde{S}$ and $\widetilde{D}_{2}$ partial wave contributions at $\sqrt{s}<1.5 \mathrm{GeV}$.

3.4. Production mechanisms of scalar resonances Expectation of the Belle data and their advent have called into being a whole series of theoretical papers which study dynamics of the $f_{0}(980)$ and $\sigma(600)$ production in the $\gamma \gamma \rightarrow \pi \pi$ processes by various means and discuss the nature of these states $[9,45,446,65,73,77$, 78, 101 105, 211, 239, 246 258.

The main lesson from the analysis of the production mechanisms of the light scalars in $\gamma \gamma$ collisions is the following [45, 46].

The classical $P$ wave tensor $q \bar{q}$ mesons $f_{2}(1270)$, $a_{2}(1320)$, and $f_{2}^{\prime}(1525)$ are produced in $\gamma \gamma$ collisions due to the direct $\gamma \gamma \rightarrow q \bar{q}$ transitions in the main, whereas the light scalar mesons $\sigma(600), f_{0}(980)$, and $a_{0}(980)$ are produced by the rescatterings $\gamma \gamma \rightarrow \pi^{+} \pi^{-} \rightarrow \sigma$, $\gamma \gamma \rightarrow K^{+} K^{-} \rightarrow f_{0}, \gamma \gamma \rightarrow\left(K^{+} K^{-}, \pi^{0} \eta\right) \rightarrow a_{0}$, and so on, i.e., due to the four quark transitions. As to the direct transitions $\gamma \gamma \rightarrow \sigma, \gamma \gamma \rightarrow f_{0}$, and $\gamma \gamma \rightarrow a_{0}$, they are strongly suppressed, as it is expected in four-quark model.

This conclusion introduces a new seminal view of the $\gamma \gamma \rightarrow \pi \pi$ reaction dynamics at low energy. Let us dwell on this point.

Recall elementary ideas of interactions of $C$ even mesons with photons based on the quark model [11, 88, 183, 184]. Coupling of the $\gamma \gamma$ system with the classical $q \bar{q}$ states, to which the light pseudoscalar $\left(J^{P C}=0^{-+}\right)$ and tensor $\left(2^{++}\right)$mesons belong, are proportional to four power of charges of constituent quarks.

Only the width of the $\pi^{0} \rightarrow \gamma \gamma$ decay is evaluated from the first principles 259262 . $\Gamma_{\pi^{0} \rightarrow \gamma \gamma}$ is determined completely by the Adler-Bell-Jackiw axial anomaly and in this case the theory (QCD) is in excellent agreement with the experiment [263, 264]. The relations between the widths of the $\pi^{0} \rightarrow \gamma \gamma, \eta \rightarrow \gamma \gamma$, and $\eta^{\prime} \rightarrow \gamma \gamma$ decays are obtained in the $q \bar{q}$ model with taking into account the effects of the $\eta-\eta^{\prime}$ mixing and the $S U(3)$ symmetry breaking [183, 262, 265].

As for the tensor mesons, in the ideal mixing case, i.e., if $f_{2}=(u \bar{u}+d \bar{d}) / \sqrt{2}$ and $f_{2}^{\prime}=s \bar{s}$, the quark model predicts the following relations for the coupling constant squared:

$$
g_{f_{2} \gamma \gamma}^{2}: g_{a_{2} \gamma \gamma}^{2}: g_{f_{2}^{\prime} \gamma \gamma}^{2}=25: 9: 2 .
$$

Though absolute values of the two-photon widths of the tensor meson decays cannot be obtained from the first principles [88, 183, 192, 203, 206, 266 268] (see also references herein), the $q \bar{q}$ model prediction (9), underlying the relations between the widths of the $f_{2}(1270) \rightarrow \gamma \gamma$, $a_{2}(1320) \rightarrow \gamma \gamma$, and $f_{2}^{\prime}(1525) \rightarrow \gamma \gamma$ decays, are used with taking into account the effects of a deviation from the ideal mixing and the $S U(3)$ symmetry breaking [11, 88, 183, 191, 269, 270]. Roughly speaking, the $q \bar{q}$ model prediction (9) is borne out by experiment. Among other things, this implies that the final state interaction effects are small, in particular, the contributions of the $f_{2}(1270) \rightarrow \pi^{+} \pi^{-} \rightarrow \gamma \gamma$ rescattering type are small in comparison with the contributions of the direct $q \bar{q}\left(2^{++}\right) \rightarrow \gamma \gamma$ transitions.

The observed smallness of the $a_{0}(980)$ and $f_{0}(980)$ meson two-photon widths in comparison with the twophoton tensor meson ones and thus the failure of the $q \bar{q}$ model prediction of the relation (4) between the widths of the direct $0^{++}$and $2^{++} \rightarrow \gamma \gamma$ transitions point to that $a_{0}(980)$ and $f_{0}(980)$ are not the quark and antiquark bound states. If the $q \bar{q}$ component is practically absent in the wave functions of the light scalars and in their $q^{2} \bar{q}^{2}$ component the white neutral vector meson pairs are practically absent too, as in the MIT bag model [85, 86], then the $\sigma(600) \rightarrow \gamma \gamma, f_{0}(980) \rightarrow \gamma \gamma$, and $a_{0}(980) \rightarrow \gamma \gamma$ decays could be the four-quark transitions caused by the rescatterings $\sigma(600) \rightarrow \pi^{+} \pi^{-} \rightarrow \gamma \gamma, \quad f_{0}(980) \rightarrow K^{+} K^{-} \rightarrow \gamma \gamma$, and $a_{0}(980) \rightarrow\left(K^{+} K^{-}, \pi^{0} \eta\right) \rightarrow \gamma \gamma$. Already in 1998 we considered such a scenario extensively [108] analyzing the Crystal Ball data [189] on the $a_{0}(980)$ resonance production in the $\gamma \gamma \rightarrow \pi^{0} \eta$ reaction; see also the discussion of the $\gamma \gamma \rightarrow K \bar{K}$ reaction mechanisms in Refs. [109, 110]. Fifteen years later, when the preliminary high statics Belle data [89] on the $f_{0}(980)$ resonance production in the $\gamma \gamma \rightarrow \pi^{+} \pi^{-}$reaction were reported, we studied what role the rescattering mechanisms, in particular, the $\gamma \gamma \rightarrow K^{+} K^{-} \rightarrow f_{0}(980) \rightarrow \pi^{+} \pi^{-}$mechanism, could play in this process [101]. As a result we showed that just this mechanism gives a reasonable scale of the $f_{0}(980)$ manifestation in the $\gamma \gamma \rightarrow \pi^{+} \pi^{-}$and $\gamma \gamma \rightarrow \pi^{0} \pi^{0}$ cross sections.

Then in the $S U(2)_{L} \times S U(2)_{R}$ linear $\sigma$ model frame we showed that the $\sigma$ field are described by its fourquark component at least in the energy (virtuality) region of the $\sigma$ resonance and the $\sigma(600)$ meson decay into $\gamma \gamma$ is the four-quark transition $\sigma(600) \rightarrow \pi^{+} \pi^{-} \rightarrow \gamma \gamma$ [9]. We also emphasized that the $\sigma$ meson contribution in the $\gamma \gamma \rightarrow \pi \pi$ amplitudes is shielded due to its strong destructive interference with the background contribu- 
tions as in the $\pi \pi \rightarrow \pi \pi$ amplitudes, ${ }^{14}$ i.e., the $\sigma$ meson is produced in the $\gamma \gamma$ collisions accompanied by the great chiral background due to the rescattering mechanism $\gamma \gamma \rightarrow \pi^{+} \pi^{-} \rightarrow(\sigma+$ background $) \rightarrow \pi \pi$, that results in the modest $\gamma \gamma \rightarrow \pi^{0} \pi^{0}$ cross section near (5-10) nb in the $\sigma$ meson region, see Fig. $8(\mathrm{~b})$. The details of this shielding are given in the next Section.

The above considerations about dynamics of the $\sigma(600), f_{0}(980)$, and $f_{2}(1270)$ resonance production were developed in analyzing the final high-statistics Belle data [102, 103] on the $\gamma \gamma \rightarrow \pi^{+} \pi^{-}$and $\gamma \gamma \rightarrow \pi^{0} \pi^{0}$ reactions, to a discussion of which we proceed.

\section{Analysis of high statistics Belle data on the reactions $\gamma \gamma \rightarrow \pi^{+} \pi^{-}$and $\gamma \gamma \rightarrow \pi^{0} \pi^{0}$. Manifestations of the $\sigma(600)$ and $f_{0}(980)$ resonances}

As noted above, the $S$ and $D_{\lambda=2}$ partial wave contributions dominate in the Born cross sections $\sigma_{0}^{\text {Born }}$ and $\sigma_{2}^{\text {Born }}$, respectively, in region of interest, $\sqrt{s}<1.5 \mathrm{GeV}$, and the $\pi \pi$ interaction is strong also in the $S$ and $D$ waves only in this region, that is why the finalstate strong interaction modifies these Born contribution in $\gamma \gamma \rightarrow \pi^{+} \pi^{-}$essentially. ${ }^{15}$ In addition, the inelastic $\gamma \gamma \rightarrow K^{+} K^{-} \rightarrow \pi \pi$ rescattering plays the important role in the $f_{0}(980)$ resonance region (for the first time this process was noted in Refs. [85, 86]).

So, we use the model for the helicity, $M_{\lambda}$, and partial, $M_{\lambda J}$, amplitudes of $\gamma \gamma \rightarrow \pi \pi$ in which the Born charged $\pi$ and $K$ exchanges modified by the strong finalstate interactions in the $S$ and $D_{2}$ waves and the di-

14 As already noted in Introduction, the presence of the large background, which shields the $\sigma$ resonance in $\pi \pi \rightarrow \pi \pi$, is a consequence of chiral symmetry.

${ }^{15}$ It is reliably established by experiment that the $S$ and $D$ wave contribution dominate in the $\pi \pi$ scattering cross sections in the isospin $I=0$ and 2 channels at $\sqrt{s}<1.5 \mathrm{GeV}$ (see, for example, data 112, 113, 115, 120, 218 220, 271, 272]). The $\pi \pi$ partial wave amplitudes $T_{J}^{I}(s)=\left\{\eta_{J}^{I}(s) \exp \left[2 i \delta_{J}^{I}(s)\right]-1\right\} /\left[2 i \rho_{\pi^{+}}(s)\right]$ with $J=0,2$ and $I=0$ (where $\delta_{J}^{I}(s)$ and $\eta_{J}^{I}(s)$ are the phase and inelasticity for the $J$ wave in the $\pi \pi$ scattering channel with the isospin $\left.I ; \rho_{\pi^{+}}(s)=\left(1-4 m_{\pi^{+}}^{2} / s\right)^{1 / 2}\right)$ reach their unitarity limits at some values of $\sqrt{s}$ in the region of interest and demonstrate both the smooth energy dependence and the sharp resonance oscillations. The $T_{2}^{0}(s)$ amplitude is dominated by the $f_{2}(1270)$ resonance contribution. The $T_{0}^{0}(s)$ amplitude contains the $\sigma_{0}(600)$ and $f_{0}(980)$ resonance contributions. The $\sigma_{0}(600)$ resonance contribution is compensated strongly by the chiral background near the $\pi \pi$ threshold to provide for the observed smallness of the $\pi \pi$ scattering length $a_{0}^{0}$ and the Adler zero in $T_{0}^{0}(s)$ at $s \approx m_{\pi}^{2} / 2[6,9,171,172] .\left|T_{0}^{0}(s)\right|$ reaches the unitary limit in the $0.85-0.9 \mathrm{GeV}$ region and has the narrow deep (practically up to zero) right under the $K \bar{K}$ threshold caused by the destructive interference of the $f_{0}(980)$ resonance contribution with the large smooth background. It is established also that the $\pi \pi$ scattering in the $I=0$ channel is elastic up to the $K \bar{K}$ channel threshold in the very good approximation, but directly above this the inelasticity $\eta_{0}^{0}(s)$ shows the sharp jump due to the production of the $f_{0}(980)$ resonance coupled strongly with the $K \bar{K}$ channel. rect transitions of the resonances in two photons are taken into account (see, in addition, 99, 101 103, 108 110, 164, 181, 247, 249, 253]),

$$
\begin{gathered}
M_{0}\left(\gamma \gamma \rightarrow \pi^{+} \pi^{-} ; s, \theta\right)=M_{0}^{\text {Born } \pi^{+}}(s, \theta)+ \\
+\widetilde{I}_{\pi^{+} \pi^{-}}^{\pi^{+}}(s) T_{\pi^{+} \pi^{-} \rightarrow \pi^{+} \pi^{-}}(s)+ \\
+\widetilde{I}_{K^{+} K^{-}}^{K^{+}}(s) T_{K^{+} K^{-} \rightarrow \pi^{+} \pi^{-}}(s)+M_{\mathrm{res}}^{\text {direct }}(s), \\
M_{2}\left(\gamma \gamma \rightarrow \pi^{+} \pi^{-} ; s, \theta\right)=M_{2}^{\text {Born } \pi^{+}}(s, \theta)+ \\
+80 \pi d_{20}^{2}(\theta) M_{\gamma \gamma \rightarrow f_{2}(1270) \rightarrow \pi^{+} \pi^{-}}(s), \\
M_{0}\left(\gamma \gamma \rightarrow \pi^{0} \pi^{0} ; s, \theta\right)=M_{00}\left(\gamma \gamma \rightarrow \pi^{0} \pi^{0} ; s\right)= \\
=\widetilde{I}_{\pi^{+}}^{\pi^{+}} \pi^{-}(s) T_{\pi^{+} \pi^{-} \rightarrow \pi^{0} \pi^{0}}(s)+ \\
+\widetilde{I}_{K^{+}}^{K^{+}} K^{-}(s) T_{K^{+} K^{-} \rightarrow \pi^{0} \pi^{0}}(s)+M_{\mathrm{res}}^{\text {direct }}(s), \\
\quad M_{2}\left(\gamma \gamma \rightarrow \pi^{0} \pi^{0} ; s, \theta\right)= \\
=5 d_{20}^{2}(\theta) M_{22}\left(\gamma \gamma \rightarrow \pi^{0} \pi^{0} ; s\right)= \\
=80 \pi d_{20}^{2}(\theta) M_{\gamma \gamma \rightarrow f_{2}(1270) \rightarrow \pi^{0} \pi^{0}}(s),
\end{gathered}
$$

where $d_{20}^{2}(\theta)=(\sqrt{6} / 4) \sin ^{2} \theta$. The diagrams of the above amplitudes are adduced in Figs. 9, 12, 13, and 14,

The first terms in the right sides of Eqs. (10) and (11) are the Born helicity amplitudes $\gamma \gamma \rightarrow \pi^{+} \pi^{-}$corresponding to the elementary one pion exchange mechanism (see Fig. 9). Their explicit forms are adduced in Appendix 8.1. The terms in Eqs. (10) and (12), containing the $T_{\pi^{+} \pi^{-} \rightarrow \pi^{+} \pi^{-}}(s)=\left[2 T_{0}^{0}(s)+\right.$ $\left.T_{0}^{2}(s)\right] / 3, \quad T_{\pi^{+} \pi^{-} \rightarrow \pi^{0} \pi^{0}}(s)=2\left[T_{0}^{0}(s)-T_{0}^{2}(s)\right] / 3, \quad$ and $T_{K^{+} K^{-} \rightarrow \pi^{+} \pi^{-}}(s)=T_{K^{+} K^{-} \rightarrow \pi^{0} \pi^{0}}(s)$ amplitudes, take into account the strong final-state interactions in the $S$ wave. Eqs. (10) and (12) imply that $T_{\pi^{+} \pi^{-} \rightarrow \pi \pi}(s)$ and $T_{K^{+} K^{-} \rightarrow \pi \pi}(s)$ in the loops of the $\gamma \gamma \rightarrow \pi^{+} \pi^{-} \rightarrow \pi \pi$ and $\gamma \gamma \rightarrow K^{+} K^{-} \rightarrow \pi \pi$ rescatterings (see Figs. 12 and 13) are on the mass shell. In so doing the $\widetilde{I}_{\pi^{+} \pi^{-}}^{\pi^{+}}(s)$ and $\widetilde{I}_{K^{+} K^{-}}^{K^{+}}(s)$ functions are the amplitudes of the triangle loop diagrams describing the transitions $\gamma \gamma \rightarrow \pi^{+} \pi^{-} \rightarrow($ scalar state with a mass $=\sqrt{s})$ and $\gamma \gamma \rightarrow K^{+} K^{-} \rightarrow($ scalar state with a mass $=\sqrt{s})$, in which the meson pairs $\pi^{+} \pi^{-}$and $K^{+} K^{-}$are produced by the electromagnetic Born sources, see Figs. 9 and 14. Their explicit forms are adduced in Appendixes 8.1 and 8.3. The amplitude $M_{\text {res }}^{\text {direct }}(s)$ in Eqs. (10) and (12) caused by the direct coupling constants of the $\sigma_{0}(600)$ and $f_{0}(980)$ with photons, and the $f_{2}(1270)$ production amplitude $M_{\gamma \gamma \rightarrow f_{2}(1270) \rightarrow \pi^{+} \pi^{-}}(s)=$ $M_{\gamma \gamma \rightarrow f_{2}(1270) \rightarrow \pi^{0} \pi^{0}}(s)$ in Eqs. (11) and (13) are specified below.

Let us show by the example of the $S$ wave amplitudes $M_{00}\left(\gamma \gamma \rightarrow \pi^{+} \pi^{-} ; s\right)$ and $M_{00}\left(\gamma \gamma \rightarrow \pi^{0} \pi^{0} ; s\right)$ that the unitary condition requirement or the Watson theorem 273 about interaction in final-state holds in the model under consideration. First of all note that the $4 \pi$ and $6 \pi$ channel contributions are small for $\sqrt{s}<1 \mathrm{GeV}$ [112 114] and consequently $T_{\pi^{+} \pi^{-} \rightarrow K^{+} K^{-}}(s)=e^{i \delta_{0}^{0}(s)}\left|T_{\pi^{+} \pi^{-} \rightarrow K^{+} K^{-}}(s)\right|$ and 


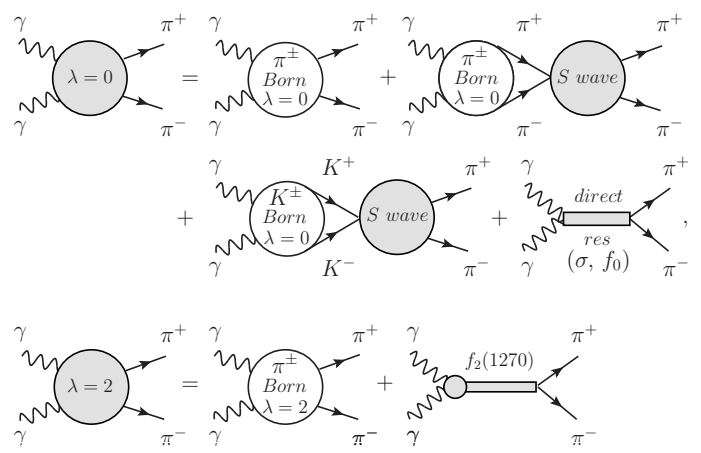

Figure 12: The diagrams corresponding to the helicity amplitudes (10) and (11) for the $\gamma \gamma \rightarrow \pi^{+} \pi^{-}$reaction.

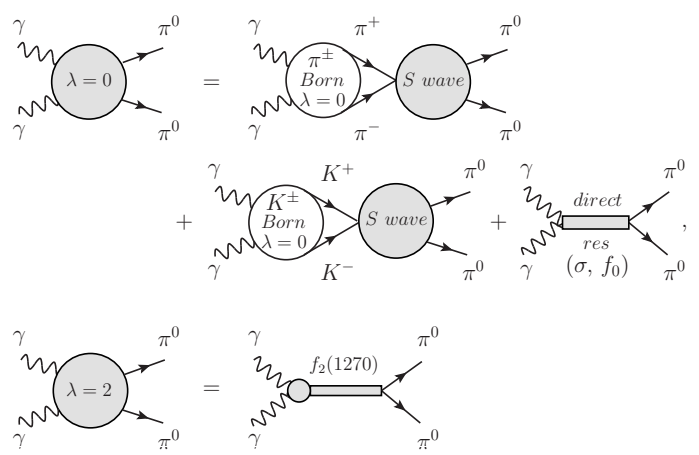

Figure 13: The diagrams corresponding to the helicity amplitudes (12) and (13) for the $\gamma \gamma \rightarrow \pi^{0} \pi^{0}$ reaction.

$M_{\text {res }}^{\text {direct }}(s)= \pm e^{i \delta_{0}^{0}(s)}\left|M_{\text {res }}^{\text {direct }}(s)\right|$ for $4 m_{\pi}^{2} \leq s \leq 4 m_{K}^{2}, \underline{9}$, 101, 102, 171, 172]. Taking into account that $\operatorname{Im} \widetilde{I}_{\pi^{+} \pi^{-}}^{\pi^{+}}(s)=\rho_{\pi^{+}}(s) M_{00}^{\text {Born } \pi^{+}}(s)$ one finds

$$
\begin{aligned}
& M_{00}\left(\gamma \gamma \rightarrow \pi^{+} \pi^{-} ; s\right)=M_{00}^{\text {Born } \pi^{+}}(s)+ \\
& +\widetilde{I}_{\pi^{+} \pi^{-}}^{\pi^{+}}(s) T_{\pi^{+} \pi^{-} \rightarrow \pi^{+} \pi^{-}}(s)+ \\
& +\widetilde{I}_{K^{+} K^{-}}^{K^{+}}(s) T_{K^{+} K^{-} \rightarrow \pi^{+} \pi^{-}}(s)+M_{\mathrm{res}}^{\text {direct }}(s)= \\
& =\left(\text { for } 2 m_{\pi} \leq \sqrt{s} \leq 2 m_{K}\right)= \\
& =\frac{2}{3} e^{i \delta_{0}^{0}(s)} A(s)+\frac{1}{3} e^{i \delta_{0}^{2}(s)} B(s), \\
& M_{00}\left(\gamma \gamma \rightarrow \pi^{0} \pi^{0} ; s\right)=\widetilde{I}_{\pi^{+} \pi^{-}}^{\pi^{+}}(s) T_{\pi^{+} \pi^{-} \rightarrow \pi^{0} \pi^{0}}(s)+ \\
& +\widetilde{I}_{K^{+} K^{-}}^{K^{+}}(s) T_{K^{+} K^{-} \rightarrow \pi^{0} \pi^{0}}(s)+M_{\mathrm{res}}^{\text {direct }}(s)= \\
& =\left(\text { for } 2 m_{\pi} \leq \sqrt{s} \leq 2 m_{K}\right)= \\
& =\frac{2}{3} e^{i \delta_{0}^{0}(s)} A(s)-\frac{2}{3} e^{i \delta_{0}^{2}(s)} B(s),
\end{aligned}
$$

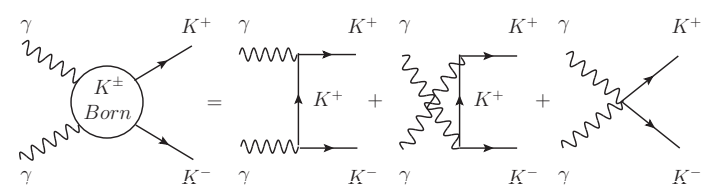

Figure 14: The Born diagrams for $\gamma \gamma \rightarrow K^{+} K^{-}$. where $\mathrm{A}(\mathrm{s})$ and $\mathrm{B}(\mathrm{s})$ are the real functions. ${ }^{16}$ Eqs. (14) and (15) show that at one with the Watson theorem the phases of the $S$ wave amplitudes $\gamma \gamma \rightarrow \pi \pi$ with $I=0$ and 2 coincide with the phases of the $\pi \pi$ scattering $\delta_{0}^{0}(s)$ and $\delta_{0}^{2}(s)$, respectively, in the elastic region (below the $K \bar{K}$ threshold).

We use the following notations and normalizations for the $\gamma \gamma \rightarrow \pi \pi$ cross sections:

$$
\begin{gathered}
\sigma\left(\gamma \gamma \rightarrow \pi^{+} \pi^{+} ;|\cos \theta| \leq 0.6\right) \equiv \sigma=\sigma_{0}+\sigma_{2} \\
\sigma\left(\gamma \gamma \rightarrow \pi^{0} \pi^{0} ;|\cos \theta| \leq 0.8\right) \equiv \tilde{\sigma}=\tilde{\sigma}_{0}+\tilde{\sigma}_{2}
\end{gathered}
$$

$$
\begin{gathered}
\sigma_{\lambda}=\frac{\rho_{\pi+}(s)}{64 \pi s} \int_{-0.6}^{0.6}\left|M_{\lambda}\left(\gamma \gamma \rightarrow \pi^{+} \pi^{-} ; s, \theta\right)\right|^{2} d \cos \theta, \\
\tilde{\sigma}_{\lambda}=\frac{\rho_{\pi+}(s)}{128 \pi s} \int_{-0.8}^{0.8}\left|M_{\lambda}\left(\gamma \gamma \rightarrow \pi^{0} \pi^{0} ; s, \theta\right)\right|^{2} d \cos \theta
\end{gathered}
$$

Hereinafter the corresponding partial cross sections will be denoted as $\sigma_{\lambda J}$ and $\tilde{\sigma}_{\lambda J}$.

Before fitting data it is helpful to center on a simplified (qualitative) scheme of their description.

In Fig. 15)(a) from Ref. [102] are adduced the theoretical curves for the cross section $\sigma=\sigma_{0}+\sigma_{2}^{\text {Born }}$ and its components $\sigma_{0}$ and $\sigma_{2}^{\text {Born }}$ corresponding to the simplest variant of the above model in which only the $S$ wave Born amplitudes $\gamma \gamma \rightarrow \pi^{+} \pi^{-}$and $\gamma \gamma \rightarrow K^{+} K^{-}$ are modified by the pion and kaon strong final-state interactions. As for all higher partial waves with $\lambda=0$ and 2, they are taken in the Born point-like approximation [101, 102]. This modification results in appearing the $f_{0}(980)$ resonance signal in $\sigma_{0}$, the value and shape of which agree very well with the Belle data, see Fig. 15(a). From comparing the corresponding curves in Figs. 15(a) and 8(a) it follows that the $S$ wave contribution to $\sigma\left(\gamma \gamma \rightarrow \pi^{+} \pi^{-} ;|\cos \theta| \leq 0.6\right)$ is small for $\sqrt{s}>0.5$ $\mathrm{GeV}$. It is clear that the $f_{2}(1270)$ resonance contribution is the main element required for the description of the Belle data on $\gamma \gamma \rightarrow \pi^{+} \pi^{-}$in the $\sqrt{s}$ region from 0.8 up to $1.5 \mathrm{GeV}$. For describing data only near the $f_{0}(980)$ resonance one can the large non-coherent background under the resonance, caused by $\sigma_{2}$, approximate by a polynomial of $\sqrt{s}$. The result of a such fit is shown in Figs. 15(c) and 15(d) 102].

By Fig. 13 and Eq. (12) taking into account the final-state interactions in the Born $\gamma \gamma \rightarrow \pi^{+} \pi^{-}$and $\gamma \gamma \rightarrow K^{+} K^{-}$amplitudes leads to the prediction of the $S$ wave amplitude of the $\gamma \gamma \rightarrow \pi^{0} \pi^{0}$ reaction [9, 101 103]. In Fig. [15(b), the $\gamma \gamma \rightarrow \pi^{0} \pi^{0}$ cross section, evaluated in the outlined above manner, are compared with the Crystal Ball and Belle data. ${ }^{17}$ In view of the fact that no

$$
\begin{aligned}
& 16 \\
& \begin{array}{c}
A(s)=M_{00}^{\operatorname{Born} \pi^{+}}(s) \cos \delta_{0}^{0}(s) \quad+ \\
\left(1 / \rho_{\pi^{+}}(s)\right) \operatorname{Re}\left[\widetilde{I}_{\pi^{+} \pi^{-}}^{+}(s)\right] \sin \delta_{0}^{0}(s)+\frac{3}{2} \widetilde{I}_{K^{+} K^{-}}^{K^{+}}(s)\left|T_{K^{+} K^{-} \rightarrow \pi^{+} \pi^{-}}(s)\right| \pm
\end{array} \\
& \frac{3}{2}\left|M_{\mathrm{res}}^{\text {direct }}(s)\right| \quad \text { and } \quad B(s)=\quad M_{00}^{\mathrm{Born} \pi^{+}}(s) \cos \delta_{0}^{2}(s) \quad+ \\
& \left(1 / \rho_{\pi^{+}}(s)\right) \operatorname{Re}\left[\widetilde{I}_{\pi^{+} \pi^{+}}^{+}(s)\right] \sin \delta_{0}^{2}(s) .
\end{aligned}
$$



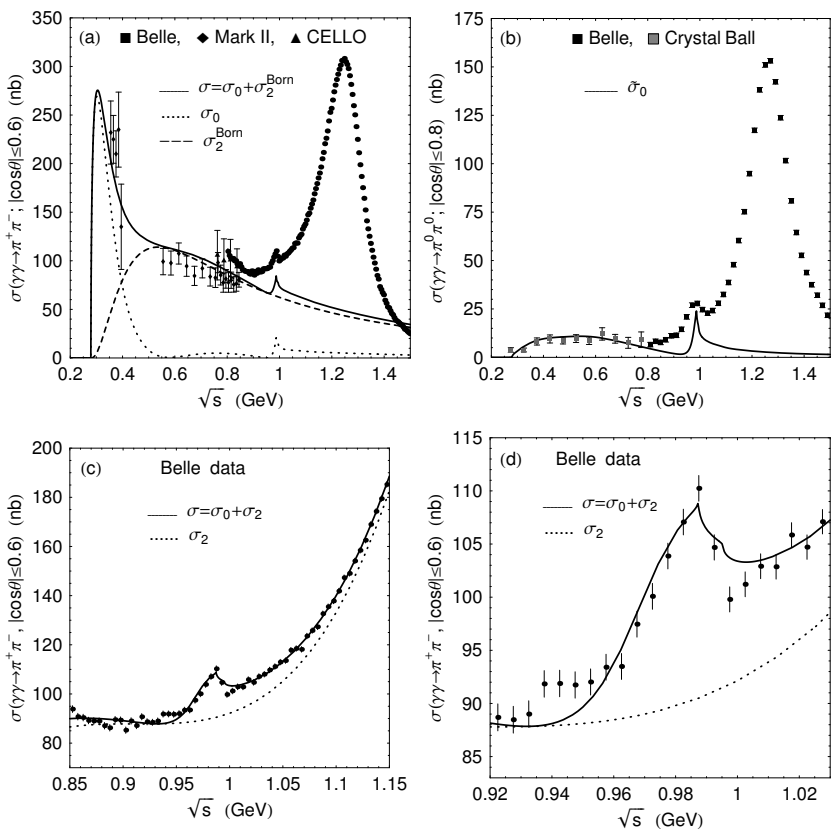

Figure 15: Theoretical curves in plots (a) and (b) correspond to the simplest model which incorporates only the Born contributions $\gamma \gamma \rightarrow \pi^{+} \pi^{-}$, from $\pi$ exchange, and $\gamma \gamma \rightarrow K^{+} K^{-}$, from $K$ exchange, modified for strong final-state interactions in the $S$ wave. Plot (c) illustrates the description of the Belle data in the $f_{0}(980)$ region. (d) The fragment of (c).

fitting parameters are used for the construction of $\tilde{\sigma}_{0}$, one should accept that the agreement with the data is rather well at $\sqrt{s} \leq 0.8 \mathrm{GeV}$, i.e., in the $\sigma(600)$ resonance region. It is clear also that at $\sqrt{s}>0.8 \mathrm{GeV}$ the $f_{2}(1270)$ resonance responsibility region begins.

So, already at this stage it emerges the following. First, if the direct coupling constants of $\sigma(600)$ and $f_{0}(980)$ with $\gamma \gamma$ are included in fitting their role will be negligible in agreement with the four-quark model prediction [85, 86]. Second, by Eqs. (10) and (12) the $\sigma(600) \rightarrow \gamma \gamma$ and $f_{0}(980) \rightarrow \gamma \gamma$ decays are described by the triangle loop rescattering diagrams Resonance $\rightarrow\left(\pi^{+} \pi^{-}\right.$, $\left.K^{+} K^{-}\right) \rightarrow \gamma \gamma$ and, consequently, are the four-quark transitions [9, 101 103].

The interesting and important feature of the $f_{0}(980)$ signal in $\gamma \gamma \rightarrow \pi^{+} \pi^{-}$is its complicated structure which is shown by Figs. 16(a) and 16(b). The $\gamma \gamma \rightarrow K^{+} K^{-} \rightarrow \pi \pi$ rescattering amplitude plays the determinant role transferring the $f_{0}(980)$ peak from the $T_{K^{+} K^{-} \rightarrow \pi \pi}(s)$ amplitude to the $\gamma \gamma \rightarrow \pi \pi$ one. ${ }^{18}$ The $\gamma \gamma \rightarrow \pi^{+} \pi^{-} \rightarrow \pi^{+} \pi^{-}$

18 It provides the natural scale of the $f_{0}(980)$ production cross section in $\gamma \gamma$ collisions 101]. The maximum of the cross section $\sigma\left(\gamma \gamma \rightarrow K^{+} K^{-} \rightarrow f_{0}(980) \rightarrow \pi^{+} \pi^{-}\right)$is controlled by the product of the ratio of the squares of the coupling constants $R_{f_{0}}=g_{f_{0} K^{+} K^{-}}^{2} / g_{f_{0} \pi^{+} \pi^{-}}^{2}$ and the value $\left|\widetilde{I}_{K^{+} K^{-}}^{K^{+}}\left(4 m_{K^{+}}^{2}\right)\right|^{2}$.
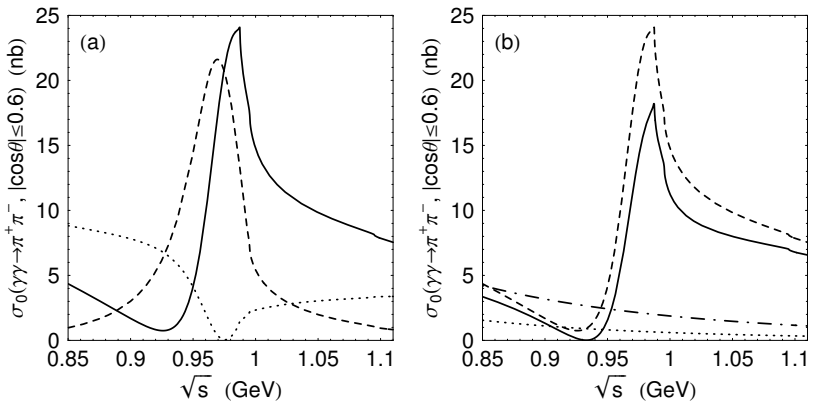

Figure 16: The structure of the $f_{0}(980)$ signal in $\sigma_{0}$. (a) The contributions from the $\gamma \gamma \rightarrow K^{+} K^{-} \rightarrow \pi^{+} \pi^{-}$(dashed line), $\gamma \gamma \rightarrow \pi^{+} \pi^{-} \rightarrow \pi^{+} \pi^{-}$(dotted line) rescattering amplitudes, and their sum (solid line). (b) The dashed line is identical to the solid one in (a), the dotted and dot-dashed lines show the $\sigma_{0}^{\text {Born }}$ and $\sigma_{00}^{\text {Born }}$ cross sections, respectively $\left(\sigma_{0}^{\text {Born }}<\sigma_{00}^{\text {Born }}\right.$ because of the destructive interference between the $S$ and higher partial waves), and the solid line corresponds to the resulting $f_{0}(980)$ signal in $\sigma_{0}$.

rescattering in its turn transfers the narrow deep under the $K \bar{K}$ threshold from the $T_{\pi \pi \rightarrow \pi \pi}(s)$ amplitude, see the footnote 18, to the $\gamma \gamma \rightarrow \pi \pi$ one. The interference of the resonance $\gamma \gamma \rightarrow K^{+} K^{-} \rightarrow \pi^{+} \pi^{-}$amplitude with the $\gamma \gamma \rightarrow \pi^{+} \pi^{-} \rightarrow \pi^{+} \pi^{-}$amplitude ${ }^{19}$ exerts the essential effect on the resulting shape of the $f_{0}(980)$ signal, as indicated by Fig. 16(a). As for the Born contributions, their influence on the resulting shape of the $f_{0}(980)$ signal is small, see Fig. 16(b).

Once more notable fact lies in the drastic change of the $f_{0}(980)$ production amplitude $\gamma \gamma \rightarrow K^{+} K^{-} \rightarrow f_{0}(980)$ in the $f_{0}(980)$ peak region [101, 102] just as the $\gamma \gamma \rightarrow K^{+} K^{-} \rightarrow a_{0}(980)$ amplitude in the $a_{0}(980)$ region [108], see Section 5. In the cross section its contribution is proportional to $\left|\widetilde{I}_{K^{+} K^{-}}^{K^{+}}(s)\right|^{2}$, see Eqs. (10) and (12). The function $\left|\widetilde{I}_{K^{+} K^{-}}^{K^{+}}(s)\right|^{2}$ decreases drastically immediately under the $K^{+} K^{-}$threshold, i.e., in the $f_{0}(980)$ resonance region, see Fig. $17{ }^{20}$ Such a behavior of the $f_{0}(980)$ two-photon production amplitude reduces strongly the left slope of the the $f_{0}(980)$ peak defined by the resonance amplitude $T_{K^{+} K^{-} \rightarrow \pi \pi}(s)$. That is why one cannot approximate the $f_{0}(980) \rightarrow \gamma \gamma$ decay width by a constant even in the region $m_{f_{0}}-\Gamma_{f_{0}} / 2 \leq \sqrt{s} \leq m_{f_{0}}+\Gamma_{f_{0}} / 2$ [101].

So, the above consideration teaches us that all simplest approximations of the $f_{0}(980)$ signal shape observed in the $\gamma \gamma \rightarrow \pi^{+} \pi^{-}$and $\gamma \gamma \rightarrow \pi^{0} \pi^{0}$ cross sections can give

Its estimate gives $\sigma\left(\gamma \gamma \rightarrow K^{+} K^{-} \rightarrow f_{0}(980) \rightarrow \pi^{+} \pi^{-} ;|\cos \theta| \leq\right.$ $0.6) \approx 0.6 \times 0.62 \alpha^{2} R_{f_{0}} / m_{f_{0}}^{2} \approx 8 \mathrm{nb} \times R_{f_{0}}$, where $\alpha=1 / 137$ and $m_{f_{0}}$ is the $f_{0}(980)$ mass.

19 Note that the relative sign between these amplitudes is fixed surely [101, 102].

20 The function $\left|\widetilde{I}_{K^{+} K^{-}}^{K^{+}}(s)\right|^{2}$ decreases relatively its maximum at $\sqrt{s}=2 m_{K^{+}} \approx 0.9873 \mathrm{GeV}$ by $1.66,2.23,2.75,3.27$, and 6.33 times at $\sqrt{s}=0.98,0.97,0.96,0.95$, and $0.9 \mathrm{GeV}$, respectively. 


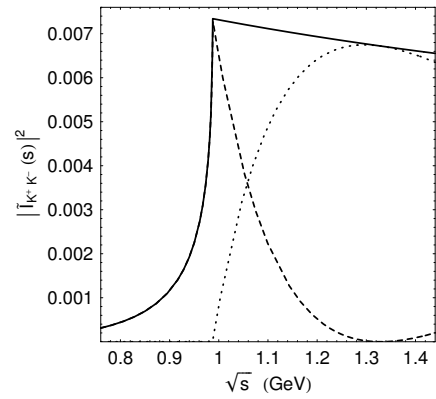

Figure 17: The solid curve shows $\left|\widetilde{I}_{K^{+} K^{-}}^{K^{+}}(s)\right|^{2}$ as a function of $\sqrt{s}$ (see Appendix 8.3); the dashed and dotted curves above the $K^{+} K^{-}$threshold correspond to the contributions of the real and imaginary parts of $\widetilde{I}_{K^{+} K^{-}}^{K^{+}}(s)$, respectively.

only a rather relative information on the $f_{0}(980)$ state two-photon production mechanism and the $f_{0}(980)$ parameters.

Fortunately, the already current knowledge of the dynamics of $T_{\pi^{+} \pi^{-} \rightarrow \pi \pi}(s)\left[T_{0}^{0}(s), T_{0}^{2}(s)\right]$ and $T_{K^{+} K^{-} \rightarrow \pi \pi}(s)$ strong interaction amplitudes allows to advance in understanding the signals about the light scalar mesons which the data on the $\gamma \gamma \rightarrow \pi \pi$ reaction send to us. In fitting data we use the model for the $T_{0}^{0}(s)$ and $T_{K^{+} K^{-} \rightarrow \pi \pi}(s)$ amplitudes which was suggested and used for the joint analysis of the data on the $\pi^{0} \pi^{0}$ mass spectrum in the $\phi \rightarrow \pi^{0} \pi^{0} \gamma$ decay, the $\pi \pi$ scattering at $2 m_{\pi}<\sqrt{s}<1.6$ $\mathrm{GeV}$, and the $\pi \pi \rightarrow K \bar{K}$ reaction [171, 172]. The $T_{0}^{0}(s)$ model takes into account the contributions of the $\sigma(600)$ and $f_{0}(980)$ resonances, their mixing, and the chiral background with the large negative phase which shields the $\sigma(600)$ resonance (see additionally [6, 9, 45, 46]). Eqs. (10) and (12) transfer the effect of the chiral shielding of the $\sigma(600)$ resonance from the $\pi \pi$ scattering into the $\gamma \gamma \rightarrow \pi \pi$ amplitudes. This effect are demonstrated by Fig. 18(a) with the help of the $\pi \pi$ scattering phases $\delta_{\text {res }}(s), \delta_{B}^{\pi \pi}(s)$, and $\delta_{0}^{0}(s)$ [see Eqs. (20)-(22) and (24)], and by Figs. 18(b) and 18(c) with the help the corresponding cross sections of the $\pi \pi \rightarrow \pi \pi$ and $\gamma \gamma \rightarrow \pi^{0} \pi^{0}$ reactions. As seen from Fig. 18(c), if it were not for such a shielding the $\gamma \gamma \rightarrow \pi^{0} \pi^{0}$ cross section nearby the threshold would be not $(5-10)$ nb but approximately 100 nb due to the $\pi^{+} \pi^{-}$loop mechanism of the $\sigma(600) \rightarrow \gamma \gamma$ decay [9]. The decay width corresponding to this mechanism, $\Gamma_{\sigma \rightarrow \pi^{+} \pi^{-} \rightarrow \gamma \gamma}(s)$, is shown in Fig. [18(d); see also Eq. (64) in Appendix 8.1.

According Refs. 171, 172], we write

$$
\begin{gathered}
T_{0}^{0}(s)=T_{B}^{\pi \pi}(s)+e^{2 i \delta_{B}^{\pi \pi}(s)} T_{\mathrm{res}}^{\pi \pi}(s), \\
T_{B}^{\pi \pi}(s)=\left\{\exp \left[2 i \delta_{B}^{\pi \pi}(s)\right]-1\right\} /\left[2 i \rho_{\pi^{+}}(s)\right], \\
T_{\mathrm{res}}^{\pi \pi}(s)=\left\{\eta_{0}^{0}(s) \exp \left[2 i \delta_{\mathrm{res}}(s)\right]-1\right\} /\left[2 i \rho_{\pi^{+}}(s)\right], \\
T_{K^{+} K^{-} \rightarrow \pi^{+} \pi^{-}}(s)=e^{i\left[\delta_{B}^{\pi \pi}(s)+\delta_{B}^{K \bar{K}}(s)\right]} T_{\mathrm{res}}^{K \bar{K} \rightarrow \pi \pi}(s),
\end{gathered}
$$

where $\delta_{B}^{\pi \pi}(s)$ and $\delta_{B}^{K \bar{K}}(s)$ are the phase of the elastic $S$ wave background in the $\pi \pi$ and $K \bar{K}$ channels with $I=0$; the $\pi \pi$ scattering phase

$$
\delta_{0}^{0}(s)=\delta_{B}^{\pi \pi}(s)+\delta_{\text {res }}(s) .
$$

The amplitudes of the $\sigma(600)-f_{0}(980)$ resonance complex in Eqs. (10), (12), (20), (22), and (23) are [171, 172]

$$
T_{\mathrm{res}}^{\pi \pi}(s)=3 \frac{g_{\sigma \pi^{+} \pi^{-}} \Delta_{f_{0}}(s)+g_{f_{0} \pi^{+} \pi^{-}} \Delta_{\sigma}(s)}{32 \pi\left[D_{\sigma}(s) D_{f_{0}}(s)-\Pi_{f_{0} \sigma}^{2}(s)\right]},
$$

$$
\begin{gathered}
T_{\mathrm{res}}^{K \bar{K} \rightarrow \pi \pi}(s)=\frac{g_{\sigma K+K}-\Delta_{f_{0}}(s)+g_{f_{0} K+K^{-}} \Delta_{\sigma}(s)}{16 \pi\left[D_{\sigma}(s) D_{f_{0}}(s)-\Pi_{f_{0} \sigma}^{2}(s)\right]}, \\
M_{\mathrm{res}}^{\text {direct }}(s)=s e^{i \delta_{B}^{\pi \pi}(s)} \frac{g_{\sigma \gamma \gamma}^{(0)} \Delta_{f_{0}}(s)+g_{f_{0} \gamma \gamma}^{(0)} \Delta_{\sigma}(s)}{D_{\sigma}(s) D_{f_{0}}(s)-\Pi_{f_{0} \sigma}^{2}(s)},
\end{gathered}
$$

where $\quad \Delta_{f_{0}}(s)=D_{f_{0}}(s) g_{\sigma \pi^{+} \pi^{-}}+\Pi_{f_{0} \sigma}(s) g_{f_{0} \pi^{+} \pi^{-}} \quad$ and $\Delta_{\sigma}(s)=D_{\sigma}(s) g_{f_{0} \pi^{+} \pi^{-}}+\Pi_{f_{0} \sigma}(s) g_{\sigma \pi^{+} \pi^{-}}, \quad g_{\sigma \gamma \gamma}^{(0)} \quad$ and $g_{f_{0} \gamma \gamma}^{(0)}$ are the direct coupling constants of the $\sigma$ and $f_{0}$ resonances with the photons. We use the expressions for the $\delta_{B}^{\pi \pi}(s)$ and $\delta_{B}^{K \bar{K}}(s)$ phases, the propagators of the $\sigma(600)$ and $f_{0}(980)$ resonances $1 / D_{\sigma}(s)$ and $1 / D_{f_{0}}(s)$, and the polarization operator matrix element $\Pi_{f_{0} \sigma}(s)$ from [171] (see also Appendix). The $m_{f_{0}}$ was free, the other parameters in the strong amplitudes $\left(m_{\sigma}, g_{\sigma \pi^{+} \pi^{-}}\right.$, $g_{f_{0} K^{+} K^{-}}$, etc.) correspond to variant 1 from Table 1 from this paper. ${ }^{21}$ We also put $\eta_{0}^{2}(s)=1$ for all $\sqrt{s}$ under consideration and take $\delta_{0}^{2}(s)$ from [274].

The amplitudes of the $f_{2}(1270)$ resonance production in Eqs. (11) and (13) are

$$
\begin{gathered}
M_{\gamma \gamma \rightarrow f_{2}(1270) \rightarrow \pi^{+} \pi^{-}}(s)=M_{\gamma \gamma \rightarrow f_{2}(1270) \rightarrow \pi^{0} \pi^{0}}(s)= \\
=\frac{\sqrt{s} G_{2}(s) \sqrt{(2 / 3) \Gamma_{f_{2} \rightarrow \pi \pi}(s) / \rho_{\pi^{+}}(s)}}{m_{f_{2}}^{2}-s-i \sqrt{s} \Gamma_{f_{2}}^{\text {tot }}(s)} .
\end{gathered}
$$

The main contribution in its total width $\Gamma_{f_{2}}^{\text {tot }}(s)=$ $\Gamma_{f_{2} \rightarrow \pi \pi}(s)+\Gamma_{f_{2} \rightarrow K \bar{K}}(s)+\Gamma_{f_{2} \rightarrow 4 \pi}(s)$ is given by the $\pi \pi$ partial decay width

$$
\begin{gathered}
\Gamma_{f_{2} \rightarrow \pi \pi}(s)=\Gamma_{f_{2}}^{\mathrm{tot}}\left(m_{f_{2}}^{2}\right) B\left(f_{2} \rightarrow \pi \pi\right) \times \\
\quad \times \frac{m_{f_{2}}^{2}}{s} \frac{q_{\pi^{+}}^{5}(s)}{q_{\pi^{+}}^{5}\left(m_{f_{2}}^{2}\right)} \frac{D_{2}\left(q_{\pi^{+}}\left(m_{f_{2}}^{2}\right) r_{f_{2}}\right)}{D_{2}\left(q_{\pi^{+}}(s) r_{f_{2}}\right)},
\end{gathered}
$$

where $D_{2}(x)=9+3 x^{2}+x^{4}, q_{\pi^{+}}(s)=\sqrt{s} \rho_{\pi^{+}}(s) / 2, r_{f_{2}}$ is the interaction range and $B\left(f_{2} \rightarrow \pi \pi\right)=0.848$ [10].

\footnotetext{
${ }^{21}$ Removing the misprint in the sign of the constant $C \equiv C_{f_{0} \sigma}$ we use $C_{f_{0} \sigma}=-0.047 \mathrm{GeV}$. Notice that our principal conclusions [the insignificance of the direct transition $\gamma \gamma \rightarrow$ Light Scalar and the dominant role of the four-quark transition $\gamma \gamma \rightarrow$ $\left(\pi^{+} \pi^{+}, K^{+} K^{-}\right) \rightarrow$ Light Scalar $]$ are independent on a specific variant from [171, 172].
} 

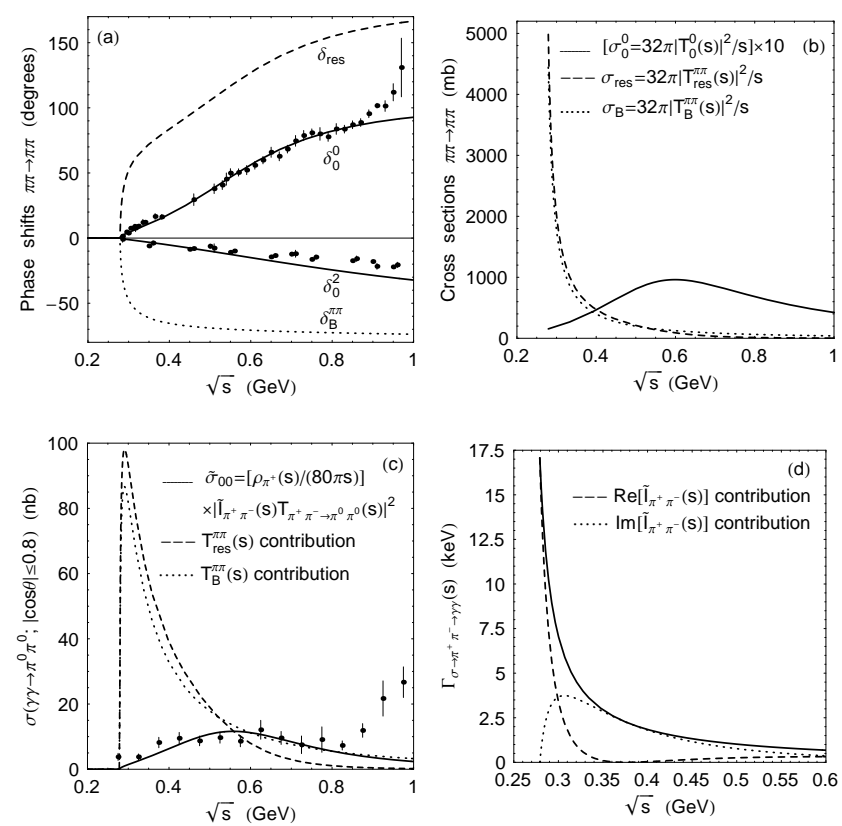

Figure 18: The figure demonstrates the chiral shielding effect in the reactions $\pi \pi \rightarrow \pi \pi$ and $\gamma \gamma \rightarrow \pi^{0} \pi^{0}$. All the plots have been taken from Ref. [9] dedicated the lightest scalar in the $S U(2)_{L} \times$ $S U(2)_{R}$ linear $\sigma$ model.

The small contributions of $\Gamma_{f_{2} \rightarrow K \bar{K}}(s)$ and $\Gamma_{f_{2} \rightarrow 4 \pi}(s)$ are the same ones as in [102]. The parameter $r_{f_{2}}$ [90, 92, 94, 95, 97, 102, 103] controls the relative form of the $f_{2}(1270)$ resonance wings and is very important especially for fitting data with small errors.

The amplitude $G_{2}(s)$ in Eq. (28) describes the coupling of the $f_{2}(1270)$ resonance with the photons,

$G_{2}(s)=\sqrt{\Gamma_{f_{2} \rightarrow \gamma \gamma}^{(0)}(s)}+i \frac{M_{22}^{\text {Born } \pi^{+}}(s)}{16 \pi} \sqrt{\frac{2}{3} \rho_{\pi^{+}}(s) \Gamma_{f_{2} \rightarrow \pi \pi}(s)}$.

The explicit form of the $M_{22}^{\text {Born }}(s)$ amplitude is in Appendix 8.1, Eq. (53). The $f_{2}(1270) \rightarrow \gamma \gamma$ decay width is

$$
\Gamma_{f_{2} \rightarrow \gamma \gamma}(s)=\left|G_{2}(s)\right|^{2}
$$

and

$$
\Gamma_{f_{2} \rightarrow \gamma \gamma}^{(0)}(s)=\frac{m_{f_{2}}}{\sqrt{s}} \Gamma_{f_{2} \rightarrow \gamma \gamma}^{(0)}\left(m_{f_{2}}^{2}\right) \frac{s^{2}}{m_{f_{2}}^{4}}
$$

[here the $s^{2}$ factor, and also the $s$ factor in Eq. (27), is caused by the gauge invariance requirement]. The second term in $G_{2}(s)$ corresponds to the rescattering $f_{2}(1270) \rightarrow \pi^{+} \pi^{-} \rightarrow \gamma \gamma$ with the real pions in intermediate state ${ }^{22}$ and ensure the fulfillment of the Watson

22 That is, it corresponds to the imaginary part of the $f_{2}(1270) \rightarrow$ $\pi^{+} \pi^{-} \rightarrow \gamma \gamma$ amplitude. theorem requirement for the amplitude $\gamma \gamma \rightarrow \pi \pi$ with $\lambda=J=2$ and $I=0$ under the first inelastic threshold. This term gives a small contribution, less then $6 \%$, in $\Gamma_{f_{2} \rightarrow \gamma \gamma}\left(m_{f_{2}}^{2}\right) .{ }^{23}$

The simplest approximation (32) of the main contribution, $\quad \Gamma_{f_{2} \rightarrow \gamma \gamma}^{(0)}(s), \quad$ in the $f_{2}(1270) \rightarrow \gamma \gamma$ decay width is completely adequate to the current state of both theory and experiment. The parameter $\Gamma_{f_{2} \rightarrow \gamma \gamma}^{(0)}\left(m_{f_{2}}^{2}\right)=\frac{1}{5}\left[g_{f_{2} \gamma \gamma}^{2} /(16 \pi)\right] m_{f_{2}}^{3}$ in Eq. (32) accumulates effectively lack of knowledge of the values of the amplitudes responsible for the $f_{2}(1270) \rightarrow \gamma \gamma$ decay. By the above reasons, see Section 3 , it is generally agreed that the direct quark-antiquark transition $q \bar{q} \rightarrow \gamma \gamma$ dominates in the $f_{2}(1270) \rightarrow \gamma \gamma$ decay and its amplitude is characterized by the $g_{f_{2} \gamma \gamma}$ coupling constant. As shown in [9, 101 106, 108] and as we state here step by step, the situation is quite different in the case of the light scalar mesons.

Now everything is ready to come to the discussion of fitting the Belle data on the $\gamma \gamma \rightarrow \pi^{+} \pi^{-}$and $\gamma \gamma \rightarrow \pi^{0} \pi^{0}$ cross sections which was carried out in [102, 103].

One considers firstly fitting the $\gamma \gamma \rightarrow \pi^{0} \pi^{0}$ cross section only, see Fig. 19(b), which has the smaller background contributions under the $f_{0}(980)$ and $f_{2}(1270)$ resonances then the $\gamma \gamma \rightarrow \pi^{+} \pi^{-}$cross section, compare Figs. 19(a) and 19(b). The solid curve in Fig. 19(b), describing these data rather well, corresponds the following parameters of the model: $m_{f_{2}}=1.269 \mathrm{GeV}, \quad \Gamma_{f_{2}}^{\text {tot }}\left(m_{f_{2}}^{2}\right)=0.182 \quad \mathrm{GeV}, \quad r_{f_{2}}=8.2$ $\mathrm{GeV}^{-1}, \quad \Gamma_{f_{2} \rightarrow \gamma \gamma}\left(m_{f_{2}}\right)=3.62 \mathrm{keV}\left[\Gamma_{f_{2} \rightarrow \gamma \gamma}^{(0)}\left(m_{f_{2}}\right)=3.43\right.$ $\mathrm{keV}], \quad m_{f_{0}}=0.969 \mathrm{GeV}, \quad g_{\sigma \gamma \gamma}^{(0)}=0.536 \quad \mathrm{GeV}^{-1}$ and $g_{f_{0} \gamma \gamma}^{(0)}=0.652 \mathrm{GeV}^{-1} \cdot{ }^{24}$ The fitting indicates smallness of the direct coupling constants $g_{\sigma \gamma \gamma}^{(0)}$ and $g_{f_{0} \gamma \gamma}^{(0)}: \quad \Gamma_{\sigma \rightarrow \gamma \gamma}^{(0)}\left(m_{\sigma}^{2}\right)=\left|m_{\sigma}^{2} g_{\sigma \gamma \gamma}^{(0)}\right|^{2} /\left(16 \pi m_{\sigma}\right)=0.012$ $\mathrm{keV}$ and $\Gamma_{f_{0} \rightarrow \gamma \gamma}^{(0)}\left(m_{f_{0}}^{2}\right)=\left|m_{f_{0}}^{2} g_{f_{0} \gamma \gamma}^{(0)}\right|^{2} /\left(16 \pi m_{f_{0}}\right)=0.008$ $\mathrm{keV}$, in accordance with the prediction [85, 86 $]^{25}$ The dominant rescattering mechanisms give the $\sigma(600) \rightarrow \pi^{+} \pi^{-} \rightarrow \gamma \gamma$ width $\approx(1-1.75) \mathrm{keV}$ averaged in the region $0.4<\sqrt{s}<0.5 \mathrm{GeV}$ [9], see Fig. 18(d), and the $f_{0}(980) \rightarrow K^{+} K^{-} \rightarrow \gamma \gamma$ width $\approx(0.15-0.2) \mathrm{keV}$

${ }^{23}$ As for a real part of the $f_{2}(1270) \rightarrow \pi^{+} \pi^{-} \rightarrow \gamma \gamma$ amplitude, its modulus is far less than the one of the direct transition amplitude as different estimations show.

24 The formally calculated errors in the significant parameters of the model are negligible due to the high statistical accuracy of the Belle data. The model dependence of adjustable parameter values is the main source of their ambiguity.

25 One notes that the small values of these coupling constants are grasped in fitting due to the interference of the $M_{\text {res }}^{\text {direct }}(s)$ amplitude, see Eqs. (10), 12), and (27), with the contributions of the dominant rescattering mechanisms. In such a case not the specific above values of $g_{\sigma \gamma \gamma}^{(0)}$ and $g_{f_{0} \gamma \gamma}^{(0)}$ are important, but the fact of their relative smallness, corresponding to $\Gamma_{\sigma \rightarrow \gamma \gamma}^{(0)}\left(m_{\sigma}^{2}\right)$ and $\Gamma_{f_{0} \rightarrow \gamma \gamma}^{(0)}\left(m_{f_{0}}^{2}\right)$ both $\ll 0.1 \mathrm{keV}$. 

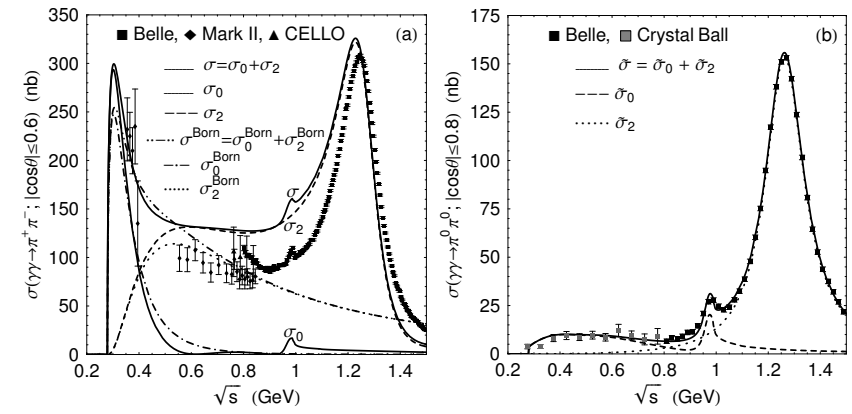

Figure 19: Cross sections for the $\gamma \gamma \rightarrow \pi^{+} \pi^{-}$and $\gamma \gamma \rightarrow \pi^{0} \pi^{0}$ reactions. Only statistical errors are shown for the Belle data 91, 92]. The curves in plot (a) are described in the text and on the figure. The curves in plot (b) are the result of the fit to the data on the $\gamma \gamma \rightarrow \pi^{0} \pi^{0}$ reaction.

averaged over the resonance mass distribution [101].

But such a fitting of the $\gamma \gamma \rightarrow \pi^{0} \pi^{0}$ cross section comes into conflict with the data on $\gamma \gamma \rightarrow \pi^{+} \pi^{-}$, see solid curve for $\sigma=\sigma_{0}+\sigma_{2}$ in Fig. 19(a). This is connected with the large Born contribution in $\sigma_{2}$ and its strong constructive (destructive) interference with the $f_{2}(1270)$ resonance contribution at $\sqrt{s}<m_{f_{2}}\left(\sqrt{s}>m_{f_{2}}\right)$, which are absent in $\gamma \gamma \rightarrow \pi^{0} \pi^{0}$. We faced this challenge in [102] and ibidem suggested the following solution. Matters can be improved by the introduction of the common cutting form factor $G_{\pi^{+}}(t, u)$ in the point-like Born amplitudes $\gamma \gamma \rightarrow \pi^{+} \pi^{-}, M_{\lambda}^{\text {Born }}(s, \theta) \rightarrow G_{\pi^{+}}(t, u) M_{\lambda}^{\text {Born }}(s, \theta)$, where $t$ and $u$ are the Mandelstam variables for the $\gamma \gamma \rightarrow \pi^{+} \pi^{-}$ reaction. ${ }^{26}$ To show this we use, as an example, the expression for $G_{\pi^{+}}(t, u)$ suggested in Ref. [191],

$G_{\pi^{+}}(t, u)=\frac{1}{s}\left[\frac{m_{\pi^{+}}^{2}-t}{1-\left(u-m_{\pi^{+}}^{2}\right) / x_{1}^{2}}+\frac{m_{\pi^{+}}^{2}-u}{1-\left(t-m_{\pi^{+}}^{2}\right) / x_{1}^{2}}\right]$,

where $x_{1}$ is a free parameter. This ansatz is quite acceptable in the physical region of the $\gamma \gamma \rightarrow \pi^{+} \pi^{-}$ reaction. Note that the form factor is introduced by changing the amplitudes of the elementary one pion exchange $M_{\lambda}^{\text {Born } \pi^{+}}(s, \theta)$ to $M_{\lambda}^{\text {Born } \pi^{+}}\left(s, \theta ; x_{1}\right)=$ $G_{\pi^{+}}(t, u) M_{\lambda}^{\text {Born } \pi^{+}}(s, \theta)$ and does not break the gauge invariance of the tree approximation [191]. Replacing in (33) $m_{\pi^{+}}$by $m_{K^{+}}$and $x_{1}$ by $x_{2}$ we obtain also the form factor $G_{K^{+}}(t, u)$ for the Born amplitudes $\gamma \gamma \rightarrow K^{+} K^{-}$.

The solid curves for $\sigma=\sigma_{0}+\sigma_{2}$ and $\tilde{\sigma}=\tilde{\sigma}_{0}+\tilde{\sigma}_{2}$ in Figs. 20(a) and 20(b) show the consistent fitting of the data on the $\gamma \gamma \rightarrow \pi^{+} \pi^{-}$cross section in the region

${ }^{26}$ Such a natural modification of the point-like Born contribution was discussed in connection with the data on the $\gamma \gamma \rightarrow \pi^{+} \pi^{-} 95$, 97, 191, 193, 194] and $\gamma \gamma \rightarrow K^{+} K^{-}\left(K^{0} \bar{K}^{0}\right)$ reactions [109, 110]. But only the problem of the consistent description of the Belle data on $\gamma \gamma \rightarrow \pi^{+} \pi^{-}$and $\gamma \gamma \rightarrow \pi^{0} \pi^{0}$ indicates the modification need of the Born sector of the model unambiguously [102, 103].
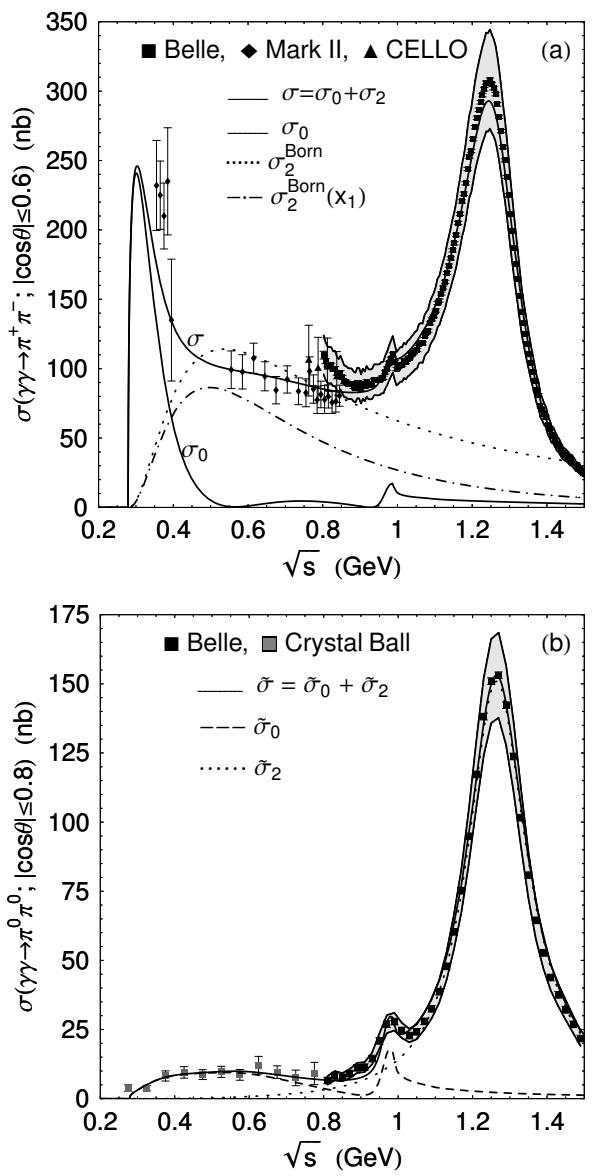

Figure 20: Joint description of the data on the cross sections for the reactions $\gamma \gamma \rightarrow \pi^{+} \pi^{-}$and $\gamma \gamma \rightarrow \pi^{0} \pi^{0}$. The shaded bands correspond to the Belle data [91, 92] with the statistical and systematic errors (errors are added quadratically). The curves are described in the text and on the figures; $\sigma_{2}{ }^{\text {Born }}\left(x_{1}\right)$ in plot (a) is the Born cross section for the $\gamma \gamma \rightarrow \pi^{+} \pi^{-}$reaction with the inclusion of the form factor.

$0.85<\sqrt{s}<1.5 \mathrm{GeV}$ and on the $\gamma \gamma \rightarrow \pi^{0} \pi^{0}$ cross section in the region $2 m_{\pi}<\sqrt{s}<1.5 \mathrm{GeV}$ with the form factors modificating the point-like Born contributions. The obtained description is more than satisfactory to within the Belle systematic errors which are shown in Figs. 20(a) and 20(b) by means of the shaded bands. We believe that such a fitting is completely adequate for the statistic errors of both Belle measurements are so small that to obtain the formally good enough value of $\chi^{2}$ in the combined fitting of the $\pi^{+} \pi^{-}$and $\pi^{0} \pi^{0}$ data in the wide regions of $\sqrt{s}$ without taking the systematic errors into consideration is practically impossible. ${ }^{27}$ The

27 At the same time we emphasize that the considerable systematic errors, the sources of which are described in detail in Refs. 9092], do not depreciate the role of the high statistics of the data, which allows to resolve the small local effects connected with the $f_{0}(980)$ resonance manifestation. 
curves in Fig. 20 correspond the following values of the parameters: $m_{f_{2}}=1.272 \mathrm{GeV}, \quad \Gamma_{f_{2}}^{\text {tot }}\left(m_{f_{2}}^{2}\right)=0.196 \mathrm{GeV}$, $r_{f_{2}}=8.2 \mathrm{GeV}^{-1}, \Gamma_{f_{2} \rightarrow \gamma \gamma}\left(m_{f_{2}}\right)=3.83 \mathrm{keV}\left[\Gamma_{f_{2} \rightarrow \gamma \gamma}^{(0)}\left(m_{f_{2}}\right)\right.$ $=3.76 \mathrm{keV}], \quad m_{f_{0}}=0.969 \mathrm{GeV}, \quad g_{\sigma \gamma \gamma}^{(0)}=-0.049 \mathrm{GeV}^{-1}$ $\left[\Gamma_{\sigma \rightarrow \gamma \gamma}^{(0)}\left(m_{\sigma}^{2}\right)\right.$ miserable $], \quad g_{f_{0} \gamma \gamma}^{(0)}=0.718 \mathrm{GeV}^{-1} \quad\left[\Gamma_{f_{0} \rightarrow \gamma \gamma}^{(0)}\right.$ $\left.\left(m_{f_{0}}^{2}\right) \approx 0.01 \mathrm{keV}\right], x_{1}=0.9 \mathrm{GeV}$ and $x_{2}=1.75 \mathrm{GeV}$. It is clear from comparison of Figs. 19(b) and 20(b) that the form factor effect on the $\gamma \gamma \rightarrow \pi^{0} \pi^{0}$ cross section is weak in contrast to the $\gamma \gamma \rightarrow \pi^{+} \pi^{-}$one [compare Figs. [19(a) and 20(a)] in which the $\sigma_{2}$ contribution is modified mainly. One emphasizes that all our conclusions about the mechanisms of the two-photon decays (productions) of the $\sigma(600)$ and $f_{0}(980)$ resonances are in force. ${ }^{28}$

Thus the physics of the two-photon decays of the light scalar mesons acquires the rather clear outline. The mechanism of their decays into $\gamma \gamma$ does not look like the mechanism of the classic tensor $q \bar{q}$ meson decays, which is the direct annihilation $q \bar{q} \rightarrow \gamma \gamma$. The light scalar meson decays into $\gamma \gamma$ are suppressed in comparison with the the tensor meson ones. They are caused by the rescattering mechanisms, i.e., by the four-quark transitions $\sigma(600) \rightarrow \pi^{+} \pi^{-} \rightarrow \gamma \gamma, \quad f_{0}(980) \rightarrow K^{+} K^{-} \rightarrow \gamma \gamma$, $a_{0}(980) \rightarrow K^{+} K^{-} \rightarrow \gamma \gamma$, etc. Such a picture is suggested by experiment and supports the $q^{2} \bar{q}^{2}$ nature of the light scalars. It is significant that in the scalar meson case the longing for the exhaustive characteristic of their coupling with photons via the constant values $\Gamma_{0^{++} \rightarrow \gamma \gamma}\left(m_{0^{++}}^{2}\right)$ by analogy with the tensor mesons cannot be realized for quite a number reasons.

First of all it is clear that when we deal with resonances accompanied by fundamental background, when two-photon decay widths change sharply in the resonance region for close inelastic thresholds, then there is no point in discussing the two-photon width in the resonance peak.

In this connection it is interesting to consider the cross section $\gamma \gamma \rightarrow \pi^{+} \pi^{-}$caused only by the resonance contributions, i.e.,

$$
\begin{gathered}
\sigma_{\mathrm{res}}\left(\gamma \gamma \rightarrow \pi^{+} \pi^{-} ; s\right)= \\
=\left[\rho_{\pi^{+}}(s) /(32 \pi s)\right] \mid \widetilde{I}_{\pi^{+} \pi^{-}}^{\pi^{+}}\left(s ; x_{1}\right) e^{2 i \delta_{B}^{\pi \pi}(s)} T_{\mathrm{res}}^{\pi \pi}(s) \\
+\widetilde{I}_{K^{+} K^{-}}^{K^{+}}\left(s ; x_{2}\right) T_{K^{+} K^{-} \rightarrow \pi^{+} \pi^{-}}(s)+\left.M_{\mathrm{res}}^{\text {direct }}(s)\right|^{2}
\end{gathered}
$$

28 Notice that the point-like $\omega$ and $a_{2}(1320)$ exchanges in the $\gamma \gamma \rightarrow \pi^{0} \pi^{0}$ and $\gamma \gamma \rightarrow \pi^{+} \pi^{-}$amplitude, respectively, which give the contributions (mainly the $S$ wave one) in the cross section, runaway with increasing the energy and comparable with the $f_{2}(1270)$ resonance contribution even in its energy region, are not observed experimentally. This was puzzled out in our paper [109] with the $\gamma \gamma \rightarrow \pi^{0} \eta$ example (the details are discussed bellow in Section 5). The proper Reggeization of the point-like exchanges with the high spins reduces the dangerous contributions greatly. In addition, the partial cancelations between the $\omega$ and $h_{1}(1170)$ exchanges in $\gamma \gamma \rightarrow \pi^{0} \pi^{0}$ and the $a_{2}(1320)$ and $a_{1}(1260)$ exchanges in $\gamma \gamma \rightarrow \pi^{+} \pi^{-}$take place. As to the $\rho$ exchange in $\gamma \gamma \rightarrow \pi \pi$, its contribution is small for $g_{\rho \pi \gamma}^{2} \approx g_{\omega \pi \gamma}^{2} / 9$ and canceled additionally by the $b_{1}(1235)$ one.

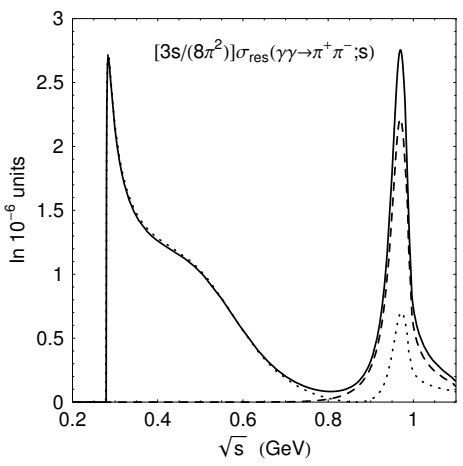

Figure 21: The integrand in Eq. 355 corresponding to the joint fit to the $\gamma \gamma \rightarrow \pi^{+} \pi^{-}$and $\gamma \gamma \rightarrow \pi^{0} \pi^{0}$ data (Fig. 20) is shown by the solid curve. The dotted and dashed curves show the contributions from the resonant elastic $\gamma \gamma \rightarrow \pi^{+} \pi^{-} \rightarrow \pi^{+} \pi^{-}$and inelastic $\gamma \gamma \rightarrow K^{+} K^{-} \rightarrow \pi^{+} \pi^{-}$rescatterings, respectively.

[see Eqs. (10) and (25)-(27)], where the functions $\widetilde{I}_{\pi^{+} \pi^{-}}^{\pi^{+}}\left(s ; x_{1}\right)$ and $\widetilde{I}_{K^{+} K^{-}}^{K^{+}}\left(s ; x_{2}\right)$ are the analogs of the $\widetilde{I}_{\pi^{+} \pi^{-}}^{\pi^{+}}(s)$ and $\widetilde{I}_{K^{+} K^{-}}^{K^{+}}(s)$ functions constructed with taking the form factors into account, see Appendixes 8.1 and 8.3. Fig. 21] shows the dependence of the $\sigma_{\text {res }}\left(\gamma \gamma \rightarrow \pi^{+} \pi^{-} ; s\right)$ cross section, multiplied by the factor $3 s /\left(8 \pi^{2}\right)$, on the energy. In and around $1 \mathrm{GeV}$ there is the impressive peak from the $f_{0}(980)$ resonance due to the inelastic $\gamma \gamma \rightarrow K^{+} K^{-} \rightarrow \pi^{+} \pi^{-}$rescattering in the main. Following Refs. 101, 103, 108, one determines the $f_{0}(980) \rightarrow \gamma \gamma$ decay width averaged over the resonance mass distribution in the $\pi \pi$ channel

$$
\left\langle\Gamma_{f_{0} \rightarrow \gamma \gamma}\right\rangle_{\pi \pi}=\int_{0.8 \mathrm{GeV}}^{1.1 \mathrm{GeV}} \frac{3 s}{8 \pi^{2}} \sigma_{\mathrm{res}}\left(\gamma \gamma \rightarrow \pi^{+} \pi^{-} ; s\right) d \sqrt{s} .
$$

This value is the adequate functional characteristic of the coupling of the $f_{0}(980)$ with $\gamma \gamma$. For the presented combined fitting, $\left\langle\Gamma_{f_{0} \rightarrow \gamma \gamma}\right\rangle_{\pi \pi} \approx 0.19 \mathrm{keV}$ [103]. Taking into account that the wide $\sigma(600)$ resonance dominates in region $2 m_{\pi}<\sqrt{s}<0.8 \mathrm{GeV}$ one obtains by analogy with (35) $\left\langle\Gamma_{\sigma \rightarrow \gamma \gamma}\right\rangle_{\pi \pi} \approx 0.45 \mathrm{keV}[103]$. Note that the cusp near the $\pi \pi$ threshold in the $\left[3 s /\left(8 \pi^{2}\right)\right] \sigma_{\text {res }}\left(\gamma \gamma \rightarrow \pi^{+} \pi^{-} ; s\right)$ expression, shown in Fig. 21, is the manifestation of the correction for the finite width in the propagator of the scalar resonance. In Appendix 8.1 there are adduced the transparent explanation of this phenomenon. In the total $S$ wave amplitude $\gamma \gamma \rightarrow \pi \pi$ such a threshold enhancement is absent due to shielding the resonance contribution in the amplitude $T_{0}^{0}(s)$ by the chiral background one, see, for example, Fig. 20(b).

The above examples, each in its manner give to feel clear the nontriviality of accessing information about the $\sigma(600)$ and $f_{0}(980)$ decays into $\gamma \gamma$. For instance, to determine $\Gamma_{\sigma \rightarrow \gamma \gamma}\left(m_{\sigma}^{2}\right)$ directly from data is impossible for the cross section in the $\sigma$ region is formed by both resonance and compensating background. The specific dynamic model of the total amplitude needs to their separation. The simple Breit-Wigner is not enough here. 
As for the $f_{0}(980)$ resonance, experimenters began to take into consideration two from three important circumstances 90 92] (see also 10, 11]), for which we drew attention in Ref. [101]. Firstly, there was taken account the correction for the finite width due to the coupling of $f_{0}(980)$ with $K \bar{K}$ channel in the $f_{0}(980)$ resonance propagator, which effects essentially on the shape of the $f_{0}(980)$ peak in the $\pi \pi$ channel. Secondly, there was taken into account the interference of the $f_{0}(980)$ resonance with the background though in the simplest form. But no model was constructed for the $f_{0}(980) \rightarrow \gamma \gamma$ decay amplitude which was approximated simply by a constant $[90-92]$. Fitting data in this way the Belle collaboration extracted the values for $\Gamma_{f_{0} \rightarrow \gamma \gamma}\left(m_{f_{0}}^{2}\right)$ presented in Table IV] But the discussion needs how to understand these values. First and foremost, one cannot use them for determining a coupling constant $g_{f_{0} \gamma \gamma}$ in a effective lagrangian, i.e., a constant of the direct transition $f_{0}(980) \rightarrow \gamma \gamma$, because such a constant is small and does not determine the $f_{0}(980) \rightarrow \gamma \gamma$ decay, as shown above. Until the model of the $f_{0}(980) \rightarrow \gamma \gamma$ decay amplitude is not specified, a meaning of the $\Gamma_{f_{0} \rightarrow \gamma \gamma}\left(m_{f_{0}}^{2}\right)$ values, extracted with the help of the simplified parametrization, is rather vague. ${ }^{29}$ In principle the $\Gamma_{f_{0} \rightarrow \gamma \gamma}\left(m_{f_{0}}^{2}\right)$ values in Table IV can be taken as the preliminary estimations of $\left\langle\Gamma_{f_{0} \rightarrow \gamma \gamma}\right\rangle$, i.e., as the $f_{0}(980) \rightarrow \gamma \gamma$ decay width averaged over the hadron mass distribution 101, 103, 108.

In the dispersion approach there are introduced usually the pole two-photon widths $\Gamma_{R \rightarrow \gamma \gamma}($ pole $), R=\sigma, f_{0}$ to characterize the coupling $\sigma(600)$ and $f_{0}(980)$ resonances with photons (see, for example, 194, 208, 222, 239, 247]). These widths are determined through the moduli of the complex pole residues of the $\gamma \gamma \rightarrow \pi \pi$ and $\pi \pi \rightarrow \pi \pi$ partial amplitudes constructed theoretically. Basing on our investigation [9] we would like to note the following. The residues of the above amplitudes are essentially complex and cannot be used as any coupling constants in a hermitian effective lagrangian. These residues are "dressed" by the background for they relate to the total amplitudes. As our analysis in the $S U(2)_{L} \times S U(2)_{R}$ linear $\sigma$-model [9] indicated, the background effects essentially on the values and phases of the residues. Thus the focus on the values of the $\Gamma_{R \rightarrow \gamma \gamma}($ pole) type in dispersion approach does not help to reveal the mechanism of the two-photon decays of the scalar mesons and so cannot shed light on the nature of the light scalars.

\section{Production of the $a_{0}(980)$ resonance in the reaction $\gamma \gamma \rightarrow \pi^{0} \eta$}

Our conclusions about the important role of the $K^{+} K^{-}$ loop mechanism in the two-photon production of the $a_{0}(980)$ resonance and its possible four-quark nature [48, 108, 109] were based on the analysis of the results

29 The above comments are true also in the $\sigma(600)$ resonance case.

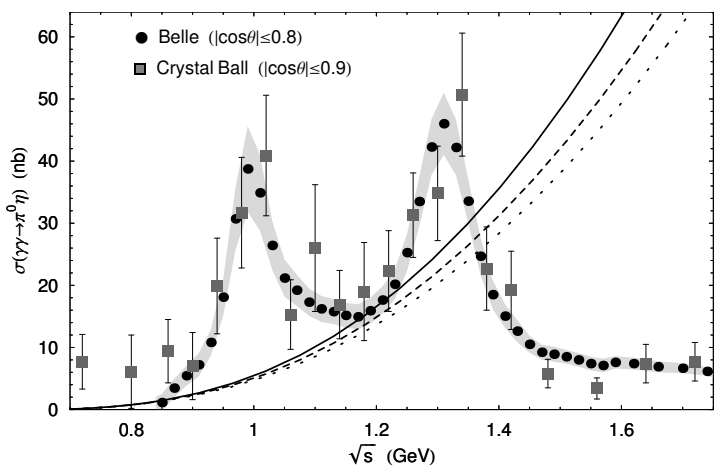

Figure 22: The Belle [93] and Crystal Ball [189] data for the $\gamma \gamma \rightarrow$ $\pi^{0} \eta$ cross section. The average statistical error of the Belle data is approximately $\pm 0.4 \mathrm{nb}$, the shaded band shows the size of their systematic error. The solid, dashed, and dotted lines correspond to the total, helicity 0 , and $S$ wave $\gamma \gamma \rightarrow \pi^{0} \eta$ cross sections caused by the elementary $\rho$ and $\omega$ exchanges for $|\cos \theta| \leq 0.8$.

of the first experiments Crystal Ball [189] (see Fig. 5) and JADE [6] on the $\gamma \gamma \rightarrow \pi^{0} \eta$ reaction. Unfortunately, the large statistical errors in these data and the rather rough step of the $\pi^{0} \eta$ invariant mass distribution (equal $40 \mathrm{MeV}$ in the Crystal Ball experiment and $60 \mathrm{MeV}$ in the JADE one) left many uncertainties.

As we have mentioned in Subsection 3.2, recently, the Belle Collaboration obtained new data on the $\gamma \gamma \rightarrow \pi^{0} \eta$ reaction at the KEKB $e^{+} e^{-}$collider [93], with statistics three orders of magnitude higher than those in the preceding Crystal Ball (336 evens) and JADE (291 events) experiments.

The experiments revealed a specific feature of the $\gamma \gamma \rightarrow \pi^{0} \eta$ cross section. It turned out sizable in the region between the $a_{0}(980)$ and $a_{2}(1320)$ resonances (see Fig. 22),${ }^{30}$ which certainly indicates the presence of additional contributions. These contributions must be coherent with the resonance ones, because only two lowest $S$ and $D_{2}$ partial waves dominate in the $\gamma \gamma \rightarrow \pi^{0} \eta$ amplitude at the invariant mass of the $\pi^{0} \eta$ system $\sqrt{s}<1.4$ $\mathrm{GeV}$ [93]. The authors of Ref. [93] performed the phenomenological fitting of the $\gamma \gamma \rightarrow \pi^{0} \eta$ data taking into account interference between resonance and background contributions. It was found that the description of the $S$ wave requires not only contributions from the $a_{0}(980)$ resonance and the possible heavy $a_{0}(Y)$ resonance, but also a smooth background whose amplitude is comparable with the amplitude of the $a_{0}(980)$ resonance at the maximum and has a large imaginary part [93]. As a result, the background results in almost the quadrupling of the cross section near the $a_{0}(980)$ peak and in the filling of the dip between the $a_{0}(980)$ and $a_{2}(1320)$ resonances. The origin of such a significant background in the $S$ wave

30 The JADE data [96] on $\gamma \gamma \rightarrow \pi^{0} \eta$ are nonnormalized and therefore are not shown in Fig. 22 
is unknown. Meanwhile, the imaginary part of the background amplitude is due to the contributions from real intermediate states, $\pi \eta, K \bar{K}$, and $\pi \eta^{\prime}$, and, naturally, requires the distinct dynamical decoding.

In Refs. [105, 106] we shown that the observed experimental pattern is a result of the interplay of many dynamical factors. To analyze the data, we significantly developed a model previously discussed in Refs. [104, 108, 109]. The basis for this model is an idea of what the $a_{0}(980)$ resonance can be as a suitable candidate in four-quark states. There exists a number of significant indications in favor of the four-quark nature of the $a_{0}(980)$; see, for example, Refs. $11,4,34,36,45$, 48, 85, 86, 133, 169]. The solution obtained by us for the $\gamma \gamma \rightarrow \pi^{0} \eta$ amplitude is in agreement with the expectations of the chiral theory for the $\pi \eta$ scattering length, with the strong coupling of the $a_{0}(980)$ resonance with the $\pi \eta, K \bar{K}$, and $\pi \eta^{\prime}$ channels, and with the key role of the $a_{0}(980) \rightarrow\left(K \bar{K}+\pi^{0} \eta+\pi^{0} \eta^{\prime}\right) \rightarrow \gamma \gamma$ rescattering mechanisms in the $a_{0}(980) \rightarrow \gamma \gamma$ decay. This picture is much in favor of the $q^{2} \bar{q}^{2}$ nature of $a_{0}(980)$ resonance and is consistent with the properties of its partners, $\sigma_{0}(600)$ and $f_{0}(980)$ resonances, in particular, with those manifested in the $\gamma \gamma \rightarrow \pi \pi$ reactions. The important role of vector exchanges in the formation of the non-resonant background in the $\gamma \gamma \rightarrow \pi^{0} \eta$ reaction has been revealed and preliminary information on the $\pi^{0} \eta \rightarrow \pi^{0} \eta$ reaction has been obtained also in Refs. [105, 106].

To analyze the Belle data, we constructed the helicity amplitudes $M_{\lambda}$ and the corresponding partial amplitudes $M_{\lambda J}$ of the $\gamma \gamma \rightarrow \pi^{0} \eta$ reaction, where the electromagnetic Born contributions from $\rho, \omega, K^{*}$, and $K$ exchanges modified by the form factors and strong elastic and inelastic final-state interactions in the $\pi^{0} \eta, \pi^{0} \eta^{\prime}, K^{+} K^{-}$, and $K^{0} \bar{K}^{0}$ channels, and the contributions from the direct interaction of the resonances with photons are taken into account:

$$
\begin{aligned}
M_{0}\left(\gamma \gamma \rightarrow \pi^{0} \eta ; s, \theta\right)=M_{0}^{\text {Born } V}\left(\gamma \gamma \rightarrow \pi^{0} \eta ; s, \theta\right)+ \\
+\widetilde{I}_{\pi^{0} \eta}^{V}(s) T_{\pi^{0} \eta \rightarrow \pi^{0} \eta}(s)+\widetilde{I}_{\pi^{0} \eta^{\prime}}^{V}(s) T_{\pi^{0} \eta^{\prime} \rightarrow \pi^{0} \eta}(s)+ \\
+\left(\widetilde{I}_{K^{+} K^{-}}^{K^{*+}}(s)-\widetilde{I}_{K^{0} \bar{K}^{0}}^{K^{* 0}}(s)+\widetilde{I}_{K^{+} K^{-}}^{K^{+}}\left(s ; x_{2}\right)\right) \times \\
\quad \times T_{K^{+} K^{-} \rightarrow \pi^{0} \eta}(s)+\widetilde{M}_{\mathrm{res}}^{\text {direct }}(s), \\
M_{2}\left(\gamma \gamma \rightarrow \pi^{0} \eta ; s, \theta\right)=M_{2}{ }^{\text {Born } V}\left(\gamma \gamma \rightarrow \pi^{0} \eta ; s, \theta\right)+ \\
+80 \pi d_{20}^{2}(\theta) M_{\gamma \gamma \rightarrow a_{2}(1320) \rightarrow \pi^{0} \eta}(s),
\end{aligned}
$$

where $\theta$ is the polar angle of the produced $\pi^{0}$ (or $\eta$ ) meson in the $\gamma \gamma$ center-of-mass system. Figs. 23 and 24 show the diagrams corresponding to these amplitudes.

The first terms in the right-hand parts of Eqs. (36) and (37) represent the real Born helicity amplitudes, which are the sums of the $\rho$ and $\omega$ exchange contributions equal in magnitude and are written in the form [108, 109]

$$
\begin{gathered}
M_{0}^{\operatorname{Born} V}\left(\gamma \gamma \rightarrow \pi^{0} \eta ; s, \theta\right)= \\
=2 g_{\omega \pi \gamma} g_{\omega \eta \gamma} \frac{s}{4}\left[\frac{t G_{\omega}(s, t)}{t-m_{\omega}^{2}}+\frac{u G_{\omega}(s, u)}{u-m_{\omega}^{2}}\right],
\end{gathered}
$$
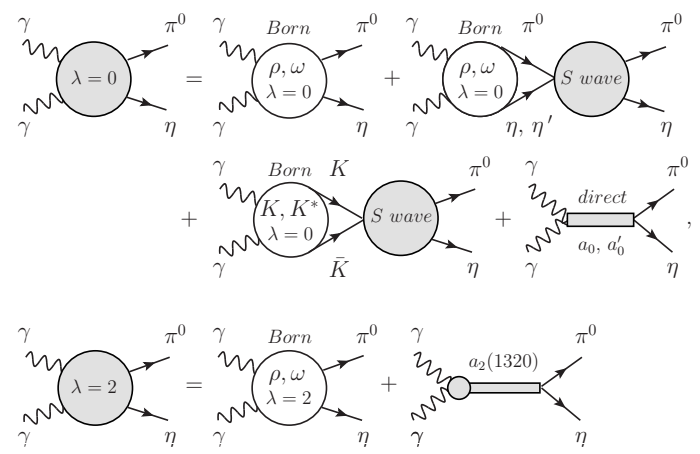

Figure 23: The diagrams corresponding to the helicity amplitudes $\gamma \gamma \rightarrow \pi^{0} \eta$, see Eqs. (36) and (37).

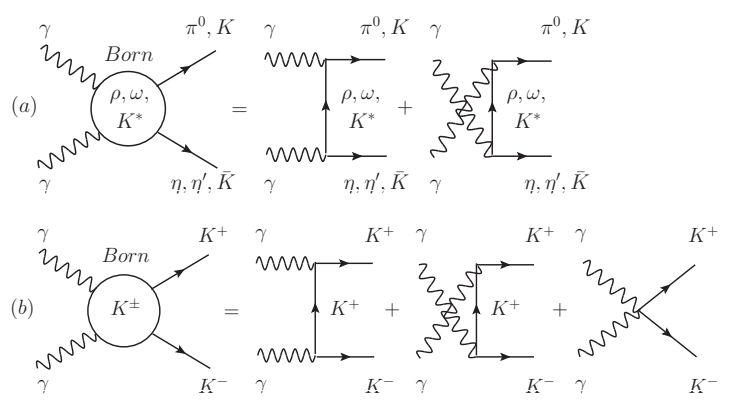

Figure 24: The Born $\rho, \omega, K^{*}$, and $K$ exchange diagrams for $\gamma \gamma \rightarrow \pi^{0} \eta, \gamma \gamma \rightarrow \pi^{0} \eta^{\prime}$, and $\gamma \gamma \rightarrow K \bar{K}$.

$$
\begin{gathered}
M_{2}^{\operatorname{Born} V}\left(\gamma \gamma \rightarrow \pi^{0} \eta ; s, \theta\right)= \\
=2 g_{\omega \pi \gamma} g_{\omega \eta \gamma} \frac{m_{\pi}^{2} m_{\eta}^{2}-t u}{4}\left[\frac{G_{\omega}(s, t)}{t-m_{\omega}^{2}}+\frac{G_{\omega}(s, u)}{u-m_{\omega}^{2}}\right]
\end{gathered}
$$

where $g_{\omega \eta \gamma}=\frac{1}{3} g_{\omega \pi \gamma} \sin \left(\theta_{i}-\theta_{P}\right), g_{\omega \pi \gamma}^{2}=12 \pi \Gamma_{\omega \rightarrow \pi \gamma}\left[\left(m_{\omega}^{2}-\right.\right.$ $\left.\left.m_{\pi}^{2}\right) /\left(2 m_{\omega}\right)\right]^{-3} \approx 0.519 \mathrm{GeV}^{-2}[10,11]$, the "ideal" mixing angle $\theta_{i}=35.3^{\circ}, \theta_{P}$ is the mixing angle in the pseudoscalar nonet, which is a free parameter; $t$ and $u$ are the Mandelstam variables for the reaction $\gamma \gamma \rightarrow$ $\pi^{0} \eta, G_{\omega}(s, t)$ and $G_{\omega}(s, u)$ are the $t$ and $u$ channel form factors [for the elementary $\rho$ and $\omega$ exchanges $\left.G_{\omega}(s, t)=G_{\omega}(s, u)=1\right]$. In the corresponding Born amplitudes for $\gamma \gamma \rightarrow \pi^{0} \eta^{\prime}, g_{\omega \eta^{\prime} \gamma}=\frac{1}{3} g_{\omega \pi \gamma} \cos \left(\theta_{i}-\theta_{P}\right)$, and for $\gamma \gamma \rightarrow K \bar{K}$ with the $K^{*}$ exchange, $g_{K^{*+} K^{+} \gamma}^{2} \approx 0.064$ $\mathrm{GeV}^{-2}$ and $g_{K^{* 0} K^{0} \gamma}^{2} \approx 0.151 \mathrm{GeV}^{-2}[10,11]$.

Note that information on the bare Born sources of the $\gamma \gamma \rightarrow \pi^{0} \eta$ reaction corresponding to the exchanges with the quantum numbers of the $\rho$ and $\omega$ mesons (as well as the b1(1235) and h1(1170) mesons) is very scarce in the nonasymptotic energy range of interest. It is known certainly only that the elementary $\rho$ and $\omega$ exchanges, whose contributions to the $\gamma \gamma \rightarrow \pi^{0} \eta$ cross section (primarily to the $\mathrm{S}$ wave) increase very rapidly with the energy, are not observed experimentally (see 
Fig. 222. ${ }^{31}$ This fact was explained in Ref. [109] by the Reggeization of the elementary exchanges, which suppresses dangerous contributions even in the range of 1$1.5 \mathrm{GeV}$. For this reason, we use the Regge type form factors $G_{\omega}(s, t)=\exp \left[\left(t-m_{\omega}^{2}\right) b_{\omega}(s)\right], G_{\omega}(s, u)=\exp [(u-$ $\left.\left.m_{\omega}^{2}\right) b_{\omega}(s)\right]$, where $b_{\omega}(s)=b_{\omega}^{0}+\left(\alpha_{\omega}^{\prime} / 4\right) \ln \left[1+\left(s / s_{0}\right)^{4}\right]$, $b_{\omega}^{0}=0, \alpha_{\omega}^{\prime}=0.8 \mathrm{GeV}^{-2}$ and $s_{0}=1 \mathrm{GeV}^{2}$ (and similar for the $K^{*}$ exchange).

As for the $b_{1}(1235)$ and $h_{1}(1170)$ exchanges, their amplitudes have the form similar to Eqs (38) and (39) except for the common sign in the amplitude with helicity 0 . The estimates show that the axial-vector exchange amplitudes are at least five times smaller than the corresponding vector exchange amplitudes and we neglect their contributions. ${ }^{32}$

The terms in Eq. (36) proportional to the $S$ wave hadron amplitudes $T_{\pi^{0} \eta \rightarrow \pi^{0} \eta}(s), T_{\pi^{0} \eta^{\prime} \rightarrow \pi^{0} \eta}(s)$, and $T_{K^{+} K^{-} \rightarrow \pi^{0} \eta}(s)$ are attributed to the rescattering mechanisms. In these amplitudes, we take into account the contribution from the mixed $a_{0}(980)$ and heavy $a_{0}(Y)$ resonances (bellow, for brevity, they are denoted as $a_{0}$ and $a_{0}^{\prime}$, respectively) and the background contributions:

$$
\begin{gathered}
T_{\pi^{0} \eta \rightarrow \pi^{0} \eta}(s)=T_{0}^{1}(s)=\frac{\eta_{0}^{1}(s) e^{2 i \delta_{0}^{1}(s)}-1}{2 i \rho_{\pi \eta}(s)}= \\
=T_{\pi \eta}^{b g}(s)+e^{2 i \delta_{\pi \eta}^{b g}(s)} T_{\pi^{0} \eta \rightarrow \pi^{0} \eta}^{r e s}(s) \\
T_{\pi^{0} \eta^{\prime} \rightarrow \pi^{0} \eta}(s)=T_{\pi^{0} \eta^{\prime} \rightarrow \pi^{0} \eta}^{r e s}(s) e^{i\left[\delta_{\pi \eta^{\prime}}^{b g}(s)+\delta_{\pi \eta}^{b g}(s)\right]}, \\
T_{K^{+} K^{-} \rightarrow \pi^{0} \eta}(s)=T_{K^{+} K^{-} \rightarrow \pi^{0} \eta}^{r e s}(s) e^{i\left[\delta_{K \bar{K}}^{b g}(s)+\delta_{\pi \eta}^{b g}(s)\right]},
\end{gathered}
$$

where $T_{\pi \eta}^{b g}(s)=\left(e^{2 i \delta_{\pi \eta}^{b g}(s)}-1\right) /\left(2 i \rho_{\pi \eta}(s)\right), T_{\pi^{0} \eta \rightarrow \pi^{0} \eta}^{r e s}(s)=$ $\left(\eta_{0}^{1}(s) e^{2 i \delta_{\pi \eta}^{r e s}(s)}-1\right) /\left(2 i \rho_{\pi \eta}(s)\right), \delta_{0}^{1}(s)=\delta_{\pi \eta}^{b g}(s)+\delta_{\pi \eta}^{r e s}(s)$, $\rho_{a b}(s)=\sqrt{s-m_{a b}^{(+)}} \sqrt{s-m_{a b}^{(-) 2}} / s, m_{a b}^{( \pm)}=m_{b} \pm m_{a}$, $a b=\pi \eta, K^{+} K^{-}, K^{0} \bar{K}^{0}, \pi \eta^{\prime} ; \delta_{\pi \eta}^{b g}(s), \delta_{\pi \eta^{\prime}}^{b g}(s)$ and $\delta_{K \bar{K}}^{b g}(s)$ are the phase shifts of the elastic background contributions in the channels $\pi \eta, \pi \eta^{\prime}$, and $K \bar{K}$ with isospin $I=1$, respectively (see Appendix 8.2).

The amplitudes of the $a_{0}-a_{0}^{\prime}$ resonance complex in Eqs. (40)-(42) have the form analogous to Eqs. (25), (26) [105, 106, 171, 275]

$$
T_{a b \rightarrow \pi^{0} \eta}^{r e s}(s)=\frac{g_{a_{0} a b} \Delta_{a_{0}^{\prime}}(s)+g_{a_{0}^{\prime} a b} \Delta_{a_{0}}(s)}{16 \pi\left[D_{a_{0}}(s) D_{a_{0}^{\prime}}(s)-\Pi_{a_{0} a_{0}^{\prime}}^{2}(s)\right]},
$$

31 These contributions are weakly sensible to the $\theta_{P}$ values under discussion [11]. The curves in Fig. [22 correspond to $\theta_{P}=-22^{\circ}$.

32 The exchanges with high spins in the $\gamma \gamma \rightarrow \pi^{0} \eta$ reaction are the correction against the background of the $K$ exchange contribution. This correction is required to describe the data for $\gamma \gamma \rightarrow \pi^{0} \eta$, as shown bellow. As to the $\gamma \gamma \rightarrow \pi \pi$ reactions, the corrections from the high spin exchanges prove to be less significant against the background of the summary contribution of the $\pi$ and $K$ exchanges, and we are unable to catch them at this stage. where $\quad \Delta_{a_{0}^{\prime}}(s)=D_{a_{0}^{\prime}}(s) g_{a_{0} \pi^{0} \eta}+\Pi_{a_{0} a_{0}^{\prime}}(s) g_{a_{0}^{\prime} \pi^{0} \eta}$ and $\quad \Delta_{a_{0}}(s)=D_{a_{0}}(s) g_{a_{0}^{\prime} \pi^{0} \eta}+\Pi_{a_{0} a_{0}^{\prime}}(s) g_{a_{0} \pi^{0} \eta}$; $g_{a_{0} a b}$ and $g_{a_{0}^{\prime} a b}$ are the coupling constants; and $1 / D_{a_{0}}(s)=1 /\left(m_{a_{0}}^{2}-s+\sum_{a b}\left[\operatorname{Re} \Pi_{a_{0}}^{a b}\left(m_{a_{0}}^{2}\right)-\Pi_{a_{0}}^{a b}(s)\right]\right)$ is the propagator for the $a_{0}$ resonance (and similar for the $a_{0}^{\prime}$ resonance), where $\operatorname{Re} \Pi_{a_{0}}^{a b}(s)$ is determined by a singly subtracted dispersion integral of $\operatorname{Im} \Pi_{a_{0}}^{a b}(s)=\sqrt{s} \Gamma_{a_{0} \rightarrow a b}(s)=g_{a_{0} a b}^{2} \rho_{a b}(s) /(16 \pi)$, $\Pi_{a_{0} a_{0}^{\prime}}(s)=C_{a_{0} a_{0}^{\prime}}+\sum_{a b}\left(g_{a_{0}^{\prime} a b} / g_{a_{0} a b}\right) \Pi_{a_{0}}^{a b}(s)$, and $C_{a_{0} a_{0}^{\prime}}$ is the resonance mixing parameter; the explicit form of the polarization operators $\Pi_{a_{0}}^{a b}(s)[104,132,169,170]$ see in Appendix 8.2. The amplitude

$$
\widetilde{M}_{\text {res }}^{\text {direct }}(s)=s \frac{g_{a_{0} \gamma \gamma}^{(0)} \Delta_{a_{0}^{\prime}}(s)+g_{a_{0}^{\prime} \gamma \gamma}^{(0)} \Delta_{a_{0}}(s)}{D_{a_{0}}(s) D_{a_{0}^{\prime}}(s)-\Pi_{a_{0} a_{0}^{\prime}}^{2}(s)} e^{i \delta_{\pi \eta}^{b g}(s)}
$$

in Eq. (36) describes the $\gamma \gamma \rightarrow \pi^{0} \eta$ transition caused by the direct coupling constants $g_{a_{0} \gamma \gamma}^{(0)}$ and $g_{a_{0}^{\prime} \gamma \gamma}^{(0)}$ of the $a_{0}$ and $a_{0}^{\prime}$ resonances with the photons; the factor $s$ appears due to the gauge invariance.

Equation (36) implies that the amplitudes $T_{a b \rightarrow \pi^{0} \eta}(s)$ in the $\gamma \gamma \rightarrow a b \rightarrow \pi^{0} \eta$ rescattering loops (see Fig. 23) are on the mass shell. In so doing, the functions $\widetilde{I}_{\pi^{0} \eta}^{V}(s)$, $\widetilde{I}_{\pi^{0} \eta^{\prime}}^{V}(s), \widetilde{I}_{K \bar{K}}^{K^{*}}(s)$, and the above mentioned function $\widetilde{I}_{K^{+} K^{-}}^{K^{+}}\left(s ; x_{2}\right)$, are the amplitudes of the triangle loop diagrams describing the transitions $\gamma \gamma \rightarrow a b \rightarrow$ (scalar state with a mass $=\sqrt{s})$, where the meson pairs $\pi^{0} \eta, \pi^{0} \eta^{\prime}$, and $K \bar{K}$ are created by electromagnetic Born sources (see Fig. 24); corresponding formulae see in Appendixes 8.2 and 8.3. The constructed amplitude $M_{0}\left(\gamma \gamma \rightarrow \pi^{0} \eta ; s, \theta\right)$ satisfies the Watson theorem in the elastic region.

For the $a_{2}(1320)$ production amplitude in (37), we use the parametrization similar to (28) and (29):

$$
=\frac{M_{\gamma \gamma \rightarrow a_{2}(1320) \rightarrow \pi^{0} \eta}(s)=}{\sqrt{s \Gamma_{a_{2} \rightarrow \gamma \gamma}(s) \Gamma_{a_{2}}^{\text {tot }}(s) B\left(a_{2} \rightarrow \pi \eta\right) / \rho_{\pi \eta}(s)}},
$$

where

$$
\Gamma_{a_{2}}^{\mathrm{tot}}(s)=\Gamma_{a_{2}}^{\mathrm{tot}} \frac{m_{a_{2}}^{2}}{s} \frac{q_{\pi \eta}^{5}(s)}{q_{\pi \eta}^{5}\left(m_{a_{2}}^{2}\right)} \frac{D_{2}\left(q_{\pi \eta}\left(m_{a_{2}}^{2}\right) r_{a_{2}}\right)}{D_{2}\left(q_{\pi \eta}(s) r_{a_{2}}\right)},
$$

$q_{\pi \eta}(s)=\sqrt{s} \rho_{\pi \eta}(s) / 2, D_{2}(x)=9+3 x^{2}+x^{4}, r_{a_{2}}$ is the interaction radius, and $\Gamma_{a_{2} \rightarrow \gamma \gamma}(s)=\left(\frac{\sqrt{s}}{m_{a_{2}}}\right)^{3} \Gamma_{a_{2} \rightarrow \gamma \gamma}$. Recall that the $f_{2}(1270) \rightarrow \gamma \gamma$ and $a_{2}(1320) \rightarrow \gamma \gamma$ decays widths rather well satisfy the relation $\Gamma_{f_{2} \rightarrow \gamma \gamma} / \Gamma_{a_{2} \rightarrow \gamma \gamma}=25 / 9$ [10, 11, 197], which is valid in the naive $q \bar{q}$ model for the direct transitions $q \bar{q} \rightarrow \gamma \gamma$.

The results of our fit to the Belle data on the $\gamma \gamma \rightarrow \pi^{0} \eta$ reaction cross section are shown in Figs. 25] and 26. The corresponding values of the model parameters are quoted in Appendix 8.2. The good agreement with the experimental data, see Fig. 25, allows for definite conclusions on the main dynamical constituents of the $\gamma \gamma \rightarrow \pi^{0} \eta$ reaction mechanism whose contributions are shown in detail in Figs. 26(a) and 26(b). 


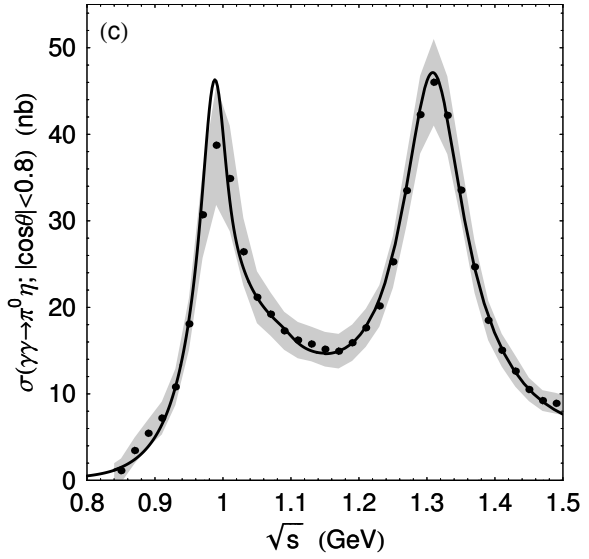

Figure 25: The fit to the Belle data on the $\gamma \gamma \rightarrow \pi^{0} \eta$ reaction cross section. The resulting solid line corresponds to the solid line 1 in Fig. 26(a) (or in Fig 26(b)), folded with a Gaussian with $\sigma=10 \mathrm{MeV}$ mass resolution; the shaded band shows the size of the systematic error of the data.

Let us begin with the contribution from the inelastic rescattering $\gamma \gamma \rightarrow K^{+} K^{-} \rightarrow \pi^{0} \eta$, where the intermediate $K^{+} K^{-}$pair is create due to the charge one kaon exchange (see Fig. 24(b)). This mechanism, as in the case of the $f_{0}(980)$ production in the $\gamma \gamma \rightarrow \pi \pi$ reactions 102, 103, specifies the natural scale for the $a_{0}(980)$ resonance production cross section in $\gamma \gamma \rightarrow \pi^{0} \eta$, and leads also to the narrowing $a_{0}(980)$ peak in this channel [104, 108]. The maximum of the cross section $\sigma\left(\gamma \gamma \rightarrow K^{+} K^{-} \rightarrow a_{0}(980) \rightarrow \pi^{0} \eta\right)$ is controlled by the product of the ratio of the squares of the coupling constants $R_{a_{0}}=g_{a_{0} K^{+} K^{-}}^{2} / g_{a_{0} \pi \eta}^{2}$ and the value $\left|\widetilde{I}_{K^{+} K^{-}}^{K^{+}}\left(4 m_{K^{+}}^{2} ; x_{2}\right)\right|^{2}$. Its estimate gives $\sigma\left(\gamma \gamma \rightarrow K^{+} K^{-} \rightarrow a_{0}(980) \rightarrow \pi^{0} \eta ;|\cos \theta| \leq 0.8\right) \approx 0.8 \times$ $1.4 \alpha^{2} R_{a_{0}} / m_{a_{0}}^{2} \approx 24 \mathrm{nb} \times R_{a_{0}}$ (here we neglect the heavy $a_{0}^{\prime}$ resonance contribution). Bellow the $K^{+} K^{-}$threshold, the function $\left|\widetilde{I}_{K^{+} K^{-}}^{K^{+}}\left(s ; x_{2}\right)\right|^{2}$ decreases sharply, resulting in the narrowing of the $a_{0}(980)$ peak in the $\gamma \gamma \rightarrow K^{+} K^{-} \rightarrow a_{0}(980) \rightarrow \pi^{0} \eta$ cross section [104, 108]. The $\gamma \gamma \rightarrow K^{+} K^{-} \rightarrow \pi^{0} \eta$ rescattering contribution to the $\gamma \gamma \rightarrow \pi^{0} \eta$ cross section is shown by solid line 3 in Fig. 26(a). The $K^{*}$ exchange also slightly narrows the $a_{0}(980)$ peak (see the dashed line under solid line 3 in Fig. 26(a)).

One $\gamma \gamma \rightarrow K \bar{K} \rightarrow \pi^{0} \eta$ rescattering mechanism is evidently insufficient to describe the data in the region of the $a_{0}(980)$ resonance. The addition of the Born contribution from the $\rho$ and $\omega$ exchanges, which is modified by the $S$ wave $\gamma \gamma \rightarrow\left(\pi^{0} \eta+\pi^{0} \eta^{\prime}\right) \rightarrow \pi^{0} \eta$ rescattering, and the amplitude $\widetilde{M}_{\text {res }}^{\text {direct }}(s)$, which is due to the direct transitions of the $a_{0}$ and $a_{0}^{\prime}$ resonances into photons, makes it possible to obtain the observed cross section magnitude. The contributions of these two mechanisms themselves are small in the region of the $a_{0}(980)$ resonance (see solid line 4 in Fig. 26(a) for the first of them and the short-dashed line in Fig. 26(b) for the second),
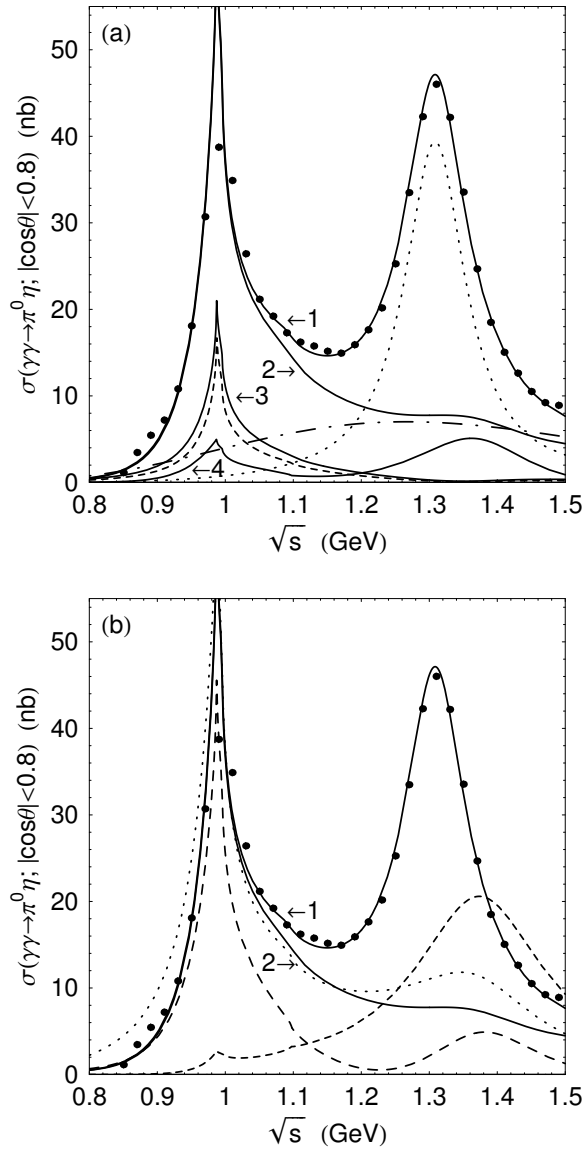

Figure 26: The fit to the Belle data. (a) Solid line 1 is the total $\gamma \gamma \rightarrow \pi^{0} \eta$ cross section, solid line 2 and the dotted line are the helicity 0 and 2 components of the cross section, solid line 3 is the contribution from the $\gamma \gamma \rightarrow K^{+} K^{-} \rightarrow \pi^{0} \eta$ rescattering with the intermediate $K+K$ - pair created due to the Born $K$ exchange, the dashed line is the contribution from the $\gamma \gamma \rightarrow K \bar{K} \rightarrow \pi^{0} \eta$ with the intermediate $K \bar{K}$ pairs created due to the Born $K$ and $K^{*}$ exchanges, the dash-dotted line is the contribution from the Born $\rho$ and $\omega$ exchanges with $\lambda=0$, and solid line 4 is the joint contribution from these exchanges and the $\mathrm{S}$ wave rescattering $\gamma \gamma \rightarrow\left(\pi^{0} \eta+\right.$ $\left.\pi^{0} \eta^{\prime}\right) \rightarrow \pi^{0} \eta$. (b) Solid lines 1 and 2 are the same as in panel (a), the short-dashed line corresponds to the contribution of the amplitude $\widetilde{M}_{\text {res }}^{\text {direct }}(s)$ caused by the direct decays of the $a_{0}$ and $a_{0}^{\prime}$ resonances into photons, the dotted line is the total contribution from the $a_{0}-a_{0}^{\prime}$ resonance complex, and the long-dashed line is the helicity 0 cross section without the contribution of the direct transition amplitude $\widetilde{M}_{\text {res }}^{\text {direct }}(s)$.

but their coherent sum with the contribution from the $\gamma \gamma \rightarrow K \bar{K} \rightarrow \pi^{0} \eta$ inelastic rescattering (see the diagrams for the amplitude with $\lambda=0$ in Fig. 23) results in the considerable enhancement of the $a_{0}(980)$ resonance (see solid line 2 in Fig. 26(a)). Recall that all the $S$ wave contributions to the $\gamma \gamma \rightarrow \pi^{0} \eta$ amplitude below the $K^{+} K^{-}$ threshold have the same phase according to the Watson theorem.

Note that, as a by-product, we extracted from the fitting of the $\gamma \gamma \rightarrow \pi^{0} \eta$ data the preliminary information on 

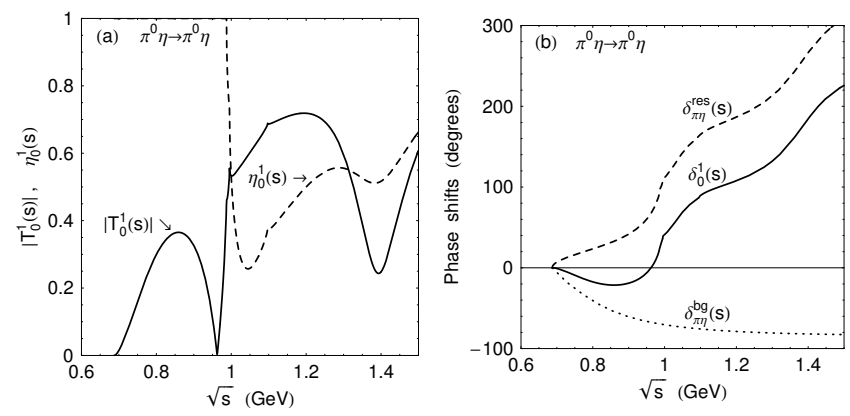

Figure 27: The $S \pi^{0} \eta \rightarrow \pi^{0} \eta$ amplitude. (a) $\left|T_{0}^{1}(s)\right|$ and inelasticity $\eta_{0}^{1}(s)$; (b) phase shifts $\left(a_{0}^{1}=0.0098\right)$.

the $S$ wave amplitude of the $\pi^{0} \eta \rightarrow \pi^{0} \eta$ reaction, which is important for the low-energy physics of pseudoscalar mesons. The characteristics for the $S$ wave amplitude $\pi^{0} \eta \rightarrow \pi^{0} \eta$ are represented in Fig. 27. Here worth noting is the important role of the background $\pi^{0} \eta$ elastic amplitude $T_{\pi \eta}^{b g}(s)$, see Eq. (40). First, the choice of the negative background phase $\delta_{\pi \eta}^{b g}(s)$ (see Fig. [27(b)) in $T_{\pi \eta}^{b g}(s)$ makes it possible to fit the $\pi \eta$ scattering length in the model under consideration to the estimates based on the current algebra [276, 277] and chiral perturbation theory [278, 279], according to which $a_{0}^{1}$ (in units of $\left.m_{\pi}^{-1}\right) \approx 0.005-0.01$. The resonance contribution $(\approx 0.3)$ to $a_{0}^{1}$ is compensated by the background contribution. Second, the significant negative value of $\delta_{\pi \eta}^{b g}(s)$ near 1 $\mathrm{GeV}$ ensures the resonance-like behavior of the cross section shown by solid line 4 in Fig. 26)(a).

We now turn to Fig. 26(b) and discuss the contribution from the possibly existing heavy $a_{0}^{\prime}$ resonance [1]]) with the mass $m_{a_{0}^{\prime}} \approx 1.4 \mathrm{GeV}$. The cross section corresponding to the amplitude $\widetilde{M}_{\text {res }}^{\text {direct }}(s)$ (see the short-dashed line) exhibits a pronounced enhancement near $1.4 \mathrm{GeV}$. In the cross section corresponding to the total contribution from the resonances (see the dotted line), i.e., from the amplitude $\widetilde{M_{\text {res }}}$ direct $(s)$ and rescattering amplitudes proportional to the amplitudes of the resonance transitions $a b \rightarrow \pi^{0} \eta\left(a b=\pi \eta, K^{+} K^{-}, K^{0} \bar{K}^{0}, \pi \eta^{\prime}\right)$, this enhancement is transferred to a shoulder. Finally, in the total cross section $\sigma_{0}$ (see solid line 2) additionally including the Born $\gamma \gamma \rightarrow \pi^{0} \eta$ contribution and the $\gamma \gamma \rightarrow \pi^{0} \eta \rightarrow \pi^{0} \eta$ rescattering caused by the background $\pi^{0} \eta \rightarrow \pi^{0} \eta$ elastic amplitude, any resonance attributes near $1.4 \mathrm{GeV}$ are absent. Thus, a strong destructive interference exists between different contributions and masks the $a_{0}^{\prime}$ resonance in the $\gamma \gamma \rightarrow \pi^{0} \eta$ cross section. Nevertheless, in many respects owing to the $a_{0}^{\prime}$, we succeed in modeling a significant smooth background under the $a_{2}(1320)$ and between $a_{0}(980)$ and $a_{2}(1320)$ resonances, which is required by the Belle data [93]. Note that due to the resulting compensations, the wide interval of $(1.28-1.42) \mathrm{GeV}$ is allowed for the mass of the $a_{0}^{\prime}$ resonance (see Ref. 105] for de- tails). ${ }^{33}$

Let us consider now the $\gamma \gamma \rightarrow \pi^{0} \eta$ cross section due only to the resonance contributions and, by analogy with Eq. (35), determine the width of the $a_{0}(980) \rightarrow \gamma \gamma$ decay averaged over the resonance mass distribution in the $\pi \eta$ channel [104, 108]:

$$
\left\langle\Gamma_{a_{0} \rightarrow \gamma \gamma}\right\rangle_{\pi \eta}=\int_{0.9 \mathrm{GeV}}^{1.1 \mathrm{GeV}} \frac{s}{4 \pi^{2}} \sigma_{\mathrm{res}}\left(\gamma \gamma \rightarrow \pi^{0} \eta ; s\right) d \sqrt{s}
$$

(the integral is calculated over the region of the $a_{0}(980)$ resonance). Taking into account the contributions from all of rescattering processes and direct decays into $\gamma \gamma$ to the $\sigma_{\text {res }}$ cross section, we obtain $\left\langle\Gamma_{a_{0} \rightarrow\left(K \bar{K}+\pi \eta+\pi \eta^{\prime}+\text { direct }\right) \rightarrow \gamma \gamma}\right\rangle_{\pi \eta} \approx 0.4 \mathrm{keV}$. Taking into account the contributions from only the rescattering processes, $\left\langle\Gamma_{a_{0} \rightarrow\left(K \bar{K}+\pi \eta+\pi \eta^{\prime}\right) \rightarrow \gamma \gamma}\right\rangle_{\pi \eta} \approx 0.23 \mathrm{keV}$, and, taking into account the contributions from only the direct decays, $\left\langle\Gamma_{a_{0} \rightarrow \gamma \gamma}^{\text {direct }}\right\rangle_{\pi \eta} \approx 0.028 \mathrm{keV}$.

The performed analysis indicates that the $a_{0}(980) \rightarrow\left(K \bar{K}+\pi^{0} \eta+\pi^{0} \eta^{\prime}\right) \rightarrow \gamma \gamma \quad$ rescattering mechanisms, i.e., the four-quark transitions, dominate in the $a_{0}(980) \rightarrow \gamma \gamma$ decay. This picture is evidence of the $q^{2} \bar{q}^{2}$ nature of the $a_{0}(980)$ resonance and is in agreement with the properties of the $\sigma_{0}(600)$ and $f_{0}(980)$ resonances, which are its partners. As to the ideal $q \bar{q}$ model prediction for the two-photon decay widths of the $f_{0}(980)$ and $a_{0}(980)$ mesons, $\Gamma_{f_{0} \rightarrow \gamma \gamma} / \Gamma_{a_{0} \rightarrow \gamma \gamma}=25 / 9$, it is excluded by experiment. ${ }^{34}$

33 Recall, that in the previous Section it was not required to introduce any heavy scalar isoscalar resonance for the theoretical description of the $\gamma \gamma \rightarrow \pi^{+} \pi^{-}$and $\gamma \gamma \rightarrow \pi^{0} \pi^{0}$ processes, as well as in Refs. [91, 92] for the phenomenological treatment of the experimental data. In principle, it could be the $f_{0}(1370)$ resonance [11]. As a matter of fact, situation with the heavy scalar resonances with the masses $\gtrsim 1.3 \mathrm{GeV}$ has been strongly tangled for a long time. For example, the authors of the review [75] seriously doubt in the existence of such a state as the $f_{0}(1370)$ (in this connection see also Refs. 280, 281]). It is possible that the wish to see the scalar resonances with the masses of (1.3-1.4) $\mathrm{GeV}$ as the partners of the well established $b_{1}(1235)$, $h_{1}(1170), a_{1}(1260), f_{1}(1285), a_{2}(1320)$, and $f_{2}(1270)$ states, belonging to the lower $P$ wave $q \bar{q}$ multiplet, is not realized in the naive way. In any case, this question remains open and requires further experimental and theoretical investigations.

${ }^{34}$ As already mentioned in Ref. [104], the model of nonrelativistic $K \bar{K}$ molecules is unjustified, because the momenta in the kaon loops describing the $\phi \rightarrow K^{+} K^{-} \rightarrow \gamma\left(f_{0} / a_{0}\right)$ and $f_{0} / a_{0} \rightarrow K^{+} K^{-} \rightarrow \gamma \gamma$ decays are high [45, 214, 215]. Our analysis gives an additional reason against the molecular model. The point is that the $a_{0}(980)$ resonance is strongly coupled with the $K \bar{K}$ and $\pi \eta$ channels, which are equivalent in the $q^{2} \bar{q}^{2}$ model. A weakly bound $K \bar{K}+\pi \eta$ molecule seems to be impossible. Moreover, the widths of the two-photon decays of the scalar resonances in the molecular model are calculated at the resonance point [282, 283], but this is insufficient for describing the $\gamma \gamma \rightarrow \pi^{+} \pi^{-}$, $\gamma \gamma \rightarrow \pi^{0} \pi^{0}$, and $\gamma \gamma \rightarrow \pi^{0} \eta$ reactions. Attempts of the description of the data on these processes in the framework of the molecular model are absent and, therefore, the results obtained in this model have the academic character. 


\section{Preliminary summary}

Results of the theoretical analysis of the experimental achievements in the low energy region, up to $1 \mathrm{GeV}$, can be formulated in the following way.

1. Naive consideration of the mass spectrum of the light scalar mesons, $\sigma(600), \kappa(800), f_{0}(980)$, and $a_{0}(980)$, gives an idea of their $q^{2} \bar{q}^{2}$ structure.

2. Both intensity and mechanism of the $a_{0}(980) / f_{0}(980)$ production in the $\phi(1020)$ meson radiative decays, the four-quark transitions $\phi(1020) \rightarrow K^{+} K^{-} \rightarrow \gamma\left[a_{0}(980) / f_{0}(980)\right]$, indicate the $q^{2} \bar{q}^{2}$ nature of the $a_{0}(980)$ and $f_{0}(980)$ states.

3. Intensities and mechanisms of the two-photon production of the light scalars, the four-quark transitions $\gamma \gamma \rightarrow \pi^{+} \pi^{-} \rightarrow \sigma(600), \gamma \gamma \rightarrow \pi^{0} \eta \rightarrow a_{0}(980)$, and $\gamma \gamma \rightarrow K^{+} K^{-} \rightarrow f_{0}(980) / a_{0}(980)$, also indicate their $q^{2} \bar{q}^{2}$ nature.

4. In addition, the absence of the $J / \psi \rightarrow \gamma f_{0}(980)$, $\rho a_{0}(980), \omega f_{0}(980)$ decays in contrast to the intensive $J / \psi \rightarrow \gamma f_{2}(1270), \quad \gamma f_{2}^{\prime}(1525), \rho a_{2}(1320)$, $\omega f_{2}(1270)$ decays intrigues against the $P$ wave $q \bar{q}$ structure of the $a_{0}(980)$ and $f_{0}(980)$ resonances.

5. It seems also undisputed that in all respects the $a_{0}(980)$ and $f_{0}(980)$ mesons are strangers in the company of the well established $b_{1}(1235), h_{1}(1170)$, $a_{1}(1260), f_{1}(1285), a_{2}(1320)$, and $f_{2}(1270)$ mesons, which are the members of the lower $P$ wave $q \bar{q}$ multiplet.

\section{Future Trends}

7.1. The $f_{0}(980)$ and $a_{0}(980)$ resonances near $\gamma \gamma \rightarrow K^{+} K^{-}$and $\gamma \gamma \rightarrow K^{0} \bar{K}^{0}$ reaction thresholds

The Belle Collaboration investigated the $\gamma \gamma \rightarrow \pi^{+} \pi^{-}$, $\gamma \gamma \rightarrow \pi^{0} \pi^{0}$, and $\gamma \gamma \rightarrow \pi^{0} \eta$ reactions with the highest statistics. ${ }^{35}$ In July 2010, the Belle Collaboration reported also the first data on the $\gamma \gamma \rightarrow \eta \eta$ reaction [285]. The $\gamma \gamma \rightarrow \eta \eta$ cross section for $\sqrt{s}>1.2 \mathrm{GeV}$ is dominated by the contributions from the tensor resonances

35 Note that high precision measurements of the $\gamma \gamma \rightarrow \pi^{+} \pi^{-}$cross section for $0.28 \mathrm{GeV}<\sqrt{s}<0.45 \mathrm{GeV}$ are planned for the KLOE2 detector at upgraded DA $\Phi$ NE $\phi$ factory in Frascati 175, 284]; the existing MARK II data 95] have in this region very large error-bars, see Fig. 6(a). Measurements of the integral and differential cross sections for $\gamma \gamma \rightarrow \pi^{+} \pi^{-}$and $\gamma \gamma \rightarrow \pi^{0} \pi^{0}$ in the $\sqrt{s}$ region from $0.45 \mathrm{GeV}$ to $1.1 \mathrm{GeV}$ [175, 284], which will complete the information from previous experiments on the $\sigma(600)$ and $f_{0}(980)$ resonance production, are also planned. In particular, the statistical uncertainty in the $\gamma \gamma \rightarrow \pi^{0} \pi^{0}$ cross section in the $\sigma(600)$ meson region (see Fig. [6(b)) will be reduced to $2 \%$.
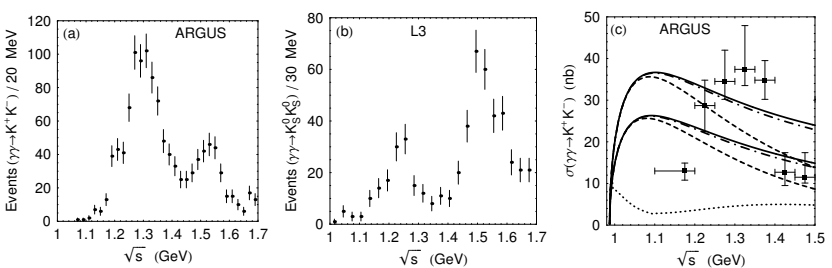

Figure 28: The $K^{+} K^{-}$(a) and $K_{S}^{0} K_{S}^{0}$ (b) mass spectra measured by ARGUS [269] and L3 291], respectively. (c) This plot illustrates the scale of the $K \bar{K}$ production cross section in $\gamma \gamma$ collisions. The experimental points show the cross section for $\gamma \gamma \rightarrow K^{+} K^{-}$with allowed contributions from $\lambda J=[22,02,00]$ [269]. The upper dashed, dot-dashed, and solid curves correspond to the $\gamma \gamma \rightarrow K^{+} K^{-}$Born cross section with $\lambda J=00, \lambda J=[00,22]$, and the total one, respectively (the $\lambda J=02$ contribution is negligible). The lower dashed, dot-dashed, and solid curves correspond to the same cross sections but modified by the form factor (see Sec. 4 and Appendix 8.3). The dotted curve shows the estimate of the the $S$ wave $\gamma \gamma \rightarrow K^{+} K^{-}$cross section in our model.

$f_{2}(1270), a_{2}(1320)$, and $f_{2}^{\prime}(1525)$. But near the threshold, $2 m_{\eta}=1.0957 \mathrm{GeV}<\sqrt{s}<1.2 \mathrm{GeV}$, there is a noticeable $S$ wave contribution, $\approx(1.5 \pm 0.15 \pm 0.7) \mathrm{nb}$, which indicates the presence of some subthreshold resonance strongly coupled with the $\eta \eta$ channel. Such a resonance in the $q^{2} \bar{q}^{2}$ model is the $f_{0}(980)$. Unfortunately, the $\gamma \gamma \rightarrow \eta \eta$ reaction is not so good for its investigation, because here only the end of the tail of this resonance can be seen.

High statistics information is still lacking for the reactions $\gamma \gamma \rightarrow K^{+} K^{-}$and $\gamma \gamma \rightarrow K^{0} \bar{K}^{0}$ in the $1 \mathrm{GeV}$ region. It is expected that the four-quark nature of the $a_{0}(980)$ and $f_{0}(980)$ resonances shows itself in these channels very originally [109, 110].

As the experiments show [100, 190, 269, 286 292], the $\gamma \gamma \rightarrow K^{+} K^{-}$and $\gamma \gamma \rightarrow K_{S}^{0} K_{S}^{0}$ cross sections in the region $1<\sqrt{s}<1.7 \mathrm{GeV}$ are saturated in fact with the contributions of the classical tensor $f_{2}(1270), a_{2}(1320)$, and $f_{2}^{\prime}(1525)$ resonances, creating in the helicity 2 states, see Fig. 28. The constructive and destructive interference between the $f_{2}(1270)$ and $a_{2}(1320)$ resonance contributions is observed in $\gamma \gamma \rightarrow K^{+} K^{-}$and $\gamma \gamma \rightarrow K^{0} \bar{K}^{0}$, respectively, in agreement with the $q \bar{q}$ model 293 . Notice that the region of the $K \bar{K}$ thresholds, $2 m_{K}<\sqrt{s}<1.1$ $\mathrm{GeV}$, sensitive to the $S$ wave contributions is not investigated in fact. The sensitivity of the ARGUS experiment to the $K^{+} K^{-}$events for $2 m_{K^{+}}<\sqrt{s}<1.1 \mathrm{GeV}$ was negligible 269], see Fig. 28(a), and the total statistics of the L3 291, see Fig. 28(b), and CLEO 100] experiments on $\gamma \gamma \rightarrow K_{S}^{0} K_{S}^{0}$ for $2 m_{K^{0}}<\sqrt{s}<1.1 \mathrm{GeV}$ are within 60 events.

The absence of the considerable non-resonance background in the $\gamma \gamma \rightarrow K^{+} K^{-}$cross section seems at first sight rather surprising for the one kaon exchange Born contribution comparable with the tensor resonance contributions, see Fig. 28(c). As seen from this figure, the $S$ wave contribution dominates in the Born cross section 
at $\sqrt{s}<1.5 \mathrm{GeV}$. One would think that the large noncoherent background should be under the tensor meson peaks in the $K^{+} K^{-}$channel. But taking into account of the resonance interaction between the $K^{+}$and $K^{-}$ mesons in the final state results in the cancelation of the considerable part of this background [109, 110]. The principal point is that the $S$ wave Born $\gamma \gamma \rightarrow K^{+} K^{-}$amplitude acquires the $\xi(s)=\left[1+i \rho_{K^{+}}(s) T_{K^{+} K^{-} \rightarrow K^{+} K^{-}}(s)\right]$ factor due to the $\gamma \gamma \rightarrow K^{+} K^{-} \rightarrow K^{+} K^{-}$rescattering amplitude with the real kaons in the intermediate state. The $a_{0}(980)$ and $f_{0}(980)$ resonance contributions dominate in the $T_{K^{+} K^{-} \rightarrow K^{+} K^{-}}(s)$ amplitude near the $K^{+} K^{-}$ threshold and provide it with the considerable imaginary part for the strong coupling with the $K \bar{K}$ channels in the four-quark scheme. As a result the $|\xi(s)|^{2}$ factor is considerably less than 1 just above the $K^{+} K^{-}$threshold and the seed $S$ wave Born contribution is considerably reduced in the wide region of $\sqrt{s}$. The dotted curve in Fig. 28(c) represents the estimation of the $S$ wave $\gamma \gamma \rightarrow K^{+} K^{-}$cross section obtained in the model under consideration (see details in Appendix 8.3). This estimation agrees with those obtained earlier [109, 110].

So one can hope to detect in the partial wave analysis of the $\gamma \gamma \rightarrow K^{+} K^{-}$reaction at $2 m_{K^{+}}<\sqrt{s}<1.1 \mathrm{GeV}$ the scalar contributions at the rate of about 5-10 nb. As for the $\gamma \gamma \rightarrow K^{0} \bar{K}^{0}$ reaction, its amplitude has not the Born contribution and the $a_{0}(980)$ resonance contribution has the opposite sign in comparison with the $\gamma \gamma \rightarrow K^{+} K^{-}$ channel. As a result the contributions of the $S$ wave $\gamma \gamma \rightarrow K^{+} K^{-} \rightarrow K^{0} \bar{K}^{0}$ rescattering amplitudes with the isotopic spin $I=0$ and 1 practically cancel each other and the the corresponding cross section should be at the rate of about $\lesssim 1 \mathrm{nb}$.

7.2. The $\sigma(600), f_{0}(980)$, and $a_{0}(980)$ resonances in $\gamma \gamma^{*}$ collisions

The investigations of light scalar mesons in the $\gamma \gamma^{*}\left(Q^{2}\right)$ collisions are promising. If $\sigma(600), f_{0}(980)$, and $a_{0}(980)$ resonances are four-quark states, their contributions to the $\gamma \gamma^{*}\left(Q^{2}\right) \rightarrow \pi^{0} \pi^{0}$ and $\gamma \gamma^{*}\left(Q^{2}\right) \rightarrow \pi^{0} \eta$ cross sections should decrease with increasing $Q^{2}$ more rapidly than the contributions from the classical tensor mesons $f_{2}(1270)$ and $a_{2}(1320)$. A similar behavior of the contribution from the exotic $q^{2} \bar{q}^{2}$ resonance state with $I^{G}\left(J^{P C}\right)=2^{+}\left(2^{++}\right)$[48, 85, 86] to the $\gamma \gamma^{*} \rightarrow \rho^{0} \rho^{0}$ and $\gamma \gamma^{*} \rightarrow \rho^{+} \rho^{-}$cross sections was recently observed by the L3 Collaboration [294 297].

\subsection{Searches for the $J / \psi \rightarrow \omega f_{0}(980)$ and $J / \psi \rightarrow$ $\rho a_{0}(980)$ decays}

These decays are important to elucidate the nature of the $f_{0}(980)$ and $a_{0}(980)$ resonances $[36,38,41]$. The $J / \psi \rightarrow$ $\rho a_{0}(980)$ decay has not been discovered as yet, $B(J / \psi \rightarrow$ $\left.\rho a_{0}(980)\right)<4.4 \times 10^{-4}[36$. As for the information on $B\left(J / \psi \rightarrow \omega f_{0}(980)\right)=(1.4 \pm 0.5) \times 10^{-4}[11]$, it would be more correctly replaced by a suitable upper limit [38, 41].

7.4. Inelasticity of $\pi \pi$ scattering and $f_{0}(980)-$ $a_{0}(980)$ mixing

By now considerable progress has been made in the experimental investigations of the $f_{0}(980)$ and $a_{0}(980)$ mesons in various reactions. Nevertheless, it turns out that equally good descriptions of the available data can be obtained for appreciably different sets of the coupling constants $g_{f_{0} K^{+} K^{-}}, g_{f_{0} \pi^{+} \pi^{-}}$, etc. (see, for example, Refs. 34, 132, 136, 171, 172]). Certainly, it would be highly desirable to fix their values. In respect of the coupling constants $g_{f_{0} K^{+} K^{-}}$and $g_{f_{0} \pi^{+} \pi^{-}}$, this question could be elucidated by precise data on the inelasticity of $\pi \pi$ scattering near the $K \bar{K}$ threshold, that have not been updated since 1975 [112 115]. It is very likely that such data in the raw form are in hand of the VES Collaboration, which was performing measurements of the $\pi^{-} p \rightarrow \pi^{+} \pi^{-} n$ reaction at IHEP (Protvino). Moreover, the product of the coupling constants $g_{a_{0} K^{+} K^{-}} g_{f_{0} K^{+} K^{-}}$may be fixed from data on the $f_{0}(980)-a_{0}(980)$ mixing, that are expected from the BESIII detector [131].

Exclusive information on $g_{a_{0} K^{+} K^{-}} g_{f_{0} K^{+} K^{-}}$can result from investigations of the spin asymmetry jump, due to the $f_{0}(980)-a_{0}(980)$ mixing, in the $\pi^{-} p \rightarrow f_{0}(980) n \rightarrow$ $a_{0}(980) n \rightarrow \pi^{0} \eta n$ reaction [124].

This work was supported in part by the RFFI Grant No. 10-02-00016 from the Russian Foundation for Basic Research.

\section{Appendix}

\section{1. $\gamma \gamma \rightarrow \pi \pi$}

Below there is the list of the expressions for the Born helicity amplitudes corresponding to the charged one pion exchange mechanism and for the triangle loop integrals $\widetilde{I}_{\pi^{+} \pi^{-}}^{\pi^{+}}(s)$ and $\widetilde{I}_{\pi^{+} \pi^{-}}^{\pi^{+}}\left(s ; x_{1}\right)$ used in Section 4. In addition, a few useful auxiliary formulae for the solitary scalar resonance are adduced.

The Born helicity amplitudes for the elementary one pion exchange in the $\gamma \gamma \rightarrow \pi^{+} \pi^{-}$reaction have the form

$$
\begin{gathered}
M_{0}^{\text {Born } \pi^{+}}(s, \theta)=\frac{4 m_{\pi^{+}}^{2}}{s} \frac{8 \pi \alpha}{1-\rho_{\pi^{+}}^{2}(s) \cos ^{2} \theta}, \\
M_{2}^{\text {Born } \pi^{+}}(s, \theta)=\frac{8 \pi \alpha \rho_{\pi^{+}}^{2}(s) \sin ^{2} \theta}{1-\rho_{\pi^{+}}^{2}(s) \cos ^{2} \theta},
\end{gathered}
$$

$\left(\rho_{\pi^{+}}(s)=\sqrt{1-4 m_{\pi^{+}}^{2} / s}\right)$. Their partial wave expansions are

$$
M_{\lambda}^{\mathrm{Born} \pi^{+}}(s, \theta)=\sum_{J \geq \lambda}(2 J+1) M_{\lambda J}^{\mathrm{Born} \pi^{+}}(s) d_{\lambda 0}^{J}(\theta),
$$

where $d_{\lambda 0}^{J}(\theta)$ are usual $d$-functions (see, for example, 10 , 11]). Three lower partial waves have the form

$$
\begin{aligned}
M_{00}^{\text {Born } \pi^{+}}(s)= & 4 \pi \alpha \frac{1-\rho_{\pi^{+}}^{2}(s)}{\rho_{\pi^{+}}(s)} \ln \frac{1+\rho_{\pi^{+}}(s)}{1-\rho_{\pi^{+}}(s)}, \\
M_{02}^{\text {Born } \pi^{+}}(s) & =4 \pi \alpha \frac{1-\rho_{\pi^{+}}^{2}(s)}{\rho_{\pi^{+}}^{2}(s)}\left[\frac{3-\rho_{\pi^{+}}^{2}(s)}{2 \rho_{\pi^{+}}(s)} \times\right. \\
& \left.\times \ln \frac{1+\rho_{\pi^{+}}(s)}{1-\rho_{\pi^{+}}(s)}-3\right],
\end{aligned}
$$




$$
\begin{aligned}
M_{22}^{\text {Born } \pi^{+}}(s)=4 \pi \alpha \sqrt{\frac{3}{2}} & {\left[\frac{\left(1-\rho_{\pi^{+}}^{2}(s)\right)^{2}}{2 \rho_{\pi^{+}}^{3}(s)} \ln \frac{1+\rho_{\pi^{+}}(s)}{1-\rho_{\pi^{+}}(s)}-\right.} \\
& \left.-\frac{1}{\rho_{\pi^{+}}^{2}(s)}+\frac{5}{3}\right]
\end{aligned}
$$

The amplitude of the triangle loop diagram, describing the transition $\gamma \gamma \rightarrow \pi^{+} \pi^{-} \rightarrow$ (scalar state with a mass $=\sqrt{s})$, is defined by

$$
\widetilde{I}_{\pi^{+} \pi^{-}}^{\pi^{+}}(s)=\frac{s}{\pi} \int_{4 m_{\pi^{+}}^{2}}^{\infty} \frac{\rho_{\pi^{+}}\left(s^{\prime}\right) M_{00}^{\mathrm{Born} \pi^{+}}\left(s^{\prime}\right)}{s^{\prime}\left(s^{\prime}-s-i \varepsilon\right)} d s^{\prime} .
$$

The behavior $\widetilde{I}_{\pi^{+} \pi^{-}}^{\pi^{+}}(s) \propto s$, when $s \rightarrow 0$, is the gauge invariance consequence. For $s \geq 4 m_{\pi^{+}}^{2}$

$$
\widetilde{I}_{\pi^{+} \pi^{-}}^{\pi^{+}}(s)=8 \alpha\left(\frac{m_{\pi^{+}}^{2}}{s}\left[\pi-2 \arctan \left|\rho_{\pi^{+}}(s)\right|\right]^{2}-1\right),
$$

for $s \geq 4 m_{\pi^{+}}^{2}$

$$
\widetilde{I}_{\pi^{+} \pi^{-}}^{\pi^{+}}(s)=8 \alpha\left\{\frac{m_{\pi^{+}}^{2}}{s}\left[\pi+i \ln \frac{1+\rho_{\pi^{+}}(s)}{1-\rho_{\pi^{+}}(s)}\right]^{2}-1\right\} .
$$

The form factor, see Eq. ([33),

$G_{\pi^{+}}(t, u)=\frac{1}{s}\left[\frac{m_{\pi^{+}}^{2}-t}{1-\left(u-m_{\pi^{+}}^{2}\right) / x_{1}^{2}}+\frac{m_{\pi^{+}}^{2}-u}{1-\left(t-m_{\pi^{+}}^{2}\right) / x_{1}^{2}}\right]$

(here $t=m_{\pi^{+}}^{2}-s\left[1-\rho_{\pi^{+}}(s) \cos \theta\right] / 2$ and $u=m_{\pi^{+}}^{2}-s[1$ $\left.\left.+\rho_{\pi^{+}}(s) \cos \theta\right] / 2\right)$ modifies the Born partial wave amplitudes. Let us introduce the notations:

$$
\begin{gathered}
M_{0 J}^{\text {Born } \pi^{+}}(s)=\frac{1-\rho_{\pi^{+}}^{2}(s)}{\rho_{\pi^{+}}(s)} F_{0 J}^{\text {Born } \pi^{+}}\left(\rho_{\pi^{+}}(s)\right), \\
M_{2 J}^{\text {Born } \pi^{+}}(s)=\rho_{\pi^{+}}(s) F_{2 J}^{\text {Born } \pi^{+}}\left(\rho_{\pi^{+}}(s)\right) .
\end{gathered}
$$

Then the modified amplitudes can be represented in the form

$$
\begin{gathered}
M_{0 J}^{\text {Born } \pi^{+}}\left(s ; x_{1}\right)=\frac{1-\rho_{\pi^{+}}^{2}(s)}{\rho_{\pi^{+}}(s)} \times \\
\times\left[F_{0 J}^{\text {Born } \pi^{+}}\left(\rho_{\pi^{+}}(s)\right)-F_{0 J}^{\text {Born } \pi^{+}}\left(\rho_{\pi^{+}}\left(s ; x_{1}\right)\right)\right], \\
M_{2 J}^{\text {Born } \pi^{+}}\left(s ; x_{1}\right)=\rho_{\pi^{+}}(s) \times \\
\times\left[F_{2 J}^{\text {Born } \pi^{+}}\left(\rho_{\pi^{+}}(s)\right)-F_{2 J}^{\text {Born } \pi^{+}}\left(\rho_{\pi^{+}}\left(s ; x_{1}\right)\right)\right],
\end{gathered}
$$

where

$$
\rho_{\pi^{+}}\left(s ; x_{1}\right)=\rho_{\pi^{+}}(s) /\left(1+2 x_{1}^{2} / s\right) .
$$

The function $\widetilde{I}_{\pi^{+} \pi^{-}}^{\pi^{+}}(s)$, see Eqs. (54)-(56), is replaced, with taking into account the form factor, by

$$
\widetilde{I}_{\pi^{+} \pi^{-}}^{\pi^{+}}\left(s ; x_{1}\right)=\frac{s}{\pi} \int_{4 m_{\pi^{+}}^{2}}^{\infty} \frac{\rho_{\pi^{+}}\left(s^{\prime}\right) M_{00}^{\mathrm{Born} \pi^{+}}\left(s^{\prime} ; x_{1}\right)}{s^{\prime}\left(s^{\prime}-s-i \varepsilon\right)} d s^{\prime} .
$$

In this case the numerical integration needs certainly.

To make easy understanding the structure and normalization of the sufficiently complicated expressions used in fitting data, one adduces the formulae for the production cross section of the $\sigma$ resonance and for its twophoton decay width due to the rescattering mechanism, $\gamma \gamma \rightarrow \pi^{+} \pi^{-} \rightarrow \sigma \rightarrow \pi^{+} \pi^{-}$, in the imaginary case of the solitary scalar $\sigma$ resonance coupled only to the $\pi \pi$ channel.

The corresponding resonance cross section has the familiar form

$$
\begin{gathered}
\sigma_{\mathrm{res}}\left(\gamma \gamma \rightarrow \pi^{+} \pi^{-} ; s\right)= \\
=\frac{8 \pi}{s} \frac{\sqrt{s} \Gamma_{\sigma \rightarrow \pi^{+} \pi^{-} \rightarrow \gamma \gamma}(s) \sqrt{s} \Gamma_{\sigma \rightarrow \pi^{+} \pi^{-}}(s)}{\left|D_{\sigma}(s)\right|^{2}}
\end{gathered}
$$

where

$$
\begin{gathered}
\Gamma_{\sigma \rightarrow \pi^{+} \pi^{-} \rightarrow \gamma \gamma}(s)=\frac{1}{16 \pi \sqrt{s}}\left|M_{\sigma \rightarrow \pi^{+} \pi^{-} \rightarrow \gamma \gamma}(s)\right|^{2}= \\
=\left|\frac{1}{16 \pi} \widetilde{I}_{\pi^{+} \pi^{-}}^{\pi^{+}}(s)\right|^{2} \frac{g_{\sigma \pi^{+} \pi^{-}}^{2}}{16 \pi \sqrt{s}} .
\end{gathered}
$$

If the $\sigma$ can else directly transit into $\gamma \gamma$ with the amplitude $s g_{\sigma \gamma \gamma}^{(0)}$ then the width $\Gamma_{\sigma \rightarrow \pi^{+} \pi^{-} \rightarrow \gamma \gamma}(s)$ in Eq. (63) should be replaced by

$$
\Gamma_{\sigma \rightarrow \gamma \gamma}(s)=\frac{1}{16 \pi \sqrt{s}}\left|M_{\sigma \rightarrow \gamma \gamma}(s)\right|^{2},
$$

where

$$
M_{\sigma \rightarrow \gamma \gamma}(s)=M_{\sigma \rightarrow \pi^{+} \pi^{-} \rightarrow \gamma \gamma}(s)+s g_{\sigma \gamma \gamma}^{(0)} .
$$

The propagator of the $\sigma$ resonance with the $m_{\sigma}$ BreitWigner mass in Eq. (63) has the form

$$
\frac{1}{D_{\sigma}(s)}=\frac{1}{m_{\sigma}^{2}-s+\operatorname{Re} \Pi_{\sigma}^{\pi \pi}\left(m_{\sigma}^{2}\right)-\Pi_{\sigma}^{\pi \pi}(s)},
$$

where $\Pi_{\sigma}^{\pi \pi}(s)$ is the polarization operator of the $\sigma$ resonance for the contribution of the $\pi^{+} \pi^{-}$and $\pi^{0} \pi^{0}$ intermediate states. For $s \geq 4 m_{\pi^{+}}^{2}\left(=4 m_{\pi^{0}}^{2}\right)$

$$
\Pi_{\sigma}^{\pi \pi}(s)=\frac{3}{2} \frac{g_{\sigma \pi^{+} \pi^{-}}^{2}}{16 \pi} \rho_{\pi^{+}}(s)\left[i-\frac{1}{\pi} \ln \frac{1+\rho_{\pi^{+}}(s)}{1-\rho_{\pi^{+}}(s)}\right] .
$$

If $0<s<4 m_{\pi^{+}}^{2}$ then $\rho_{\pi^{+}}(s) \rightarrow i\left|\rho_{\pi^{+}}(s)\right|$ and

$$
\Pi_{\sigma}^{\pi \pi}(s)=-\frac{3}{2} \frac{g_{\sigma \pi^{+} \pi^{-}}^{2}}{16 \pi}\left|\rho_{\pi^{+}}(s)\right|\left[1-\frac{2}{\pi} \arctan \left|\rho_{\pi^{+}}(s)\right|\right] .
$$

The $\sigma \rightarrow \pi \pi$ decay width is

$$
\Gamma_{\sigma \rightarrow \pi \pi}(s)=\frac{1}{\sqrt{s}} \operatorname{Im} \Pi_{\sigma}^{\pi \pi}(s)=\frac{3}{2} \frac{g_{\sigma \pi^{+} \pi^{-}}^{2}}{16 \pi} \frac{\rho_{\pi^{+}}(s)}{\sqrt{s}} .
$$

The function $\operatorname{Re}\left[\Pi_{\sigma}^{\pi \pi}\left(m_{\sigma}^{2}\right)-\Pi_{\sigma}^{\pi \pi}(s)\right]$ in the denominator of Eq. (67) is the correction for the finite width of the resonance. In Fig. 29 the real and imaginary 


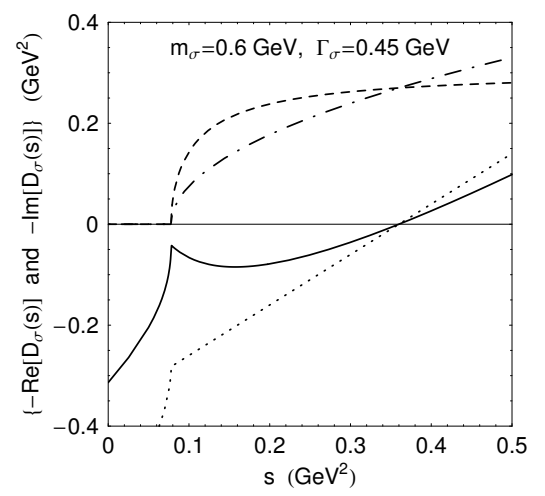

Figure 29: Demonstration of the finite width correction with an example of the single $\sigma$ resonance. The curves are described in the text.

parts of the inverse propagator $D_{\sigma}(s)$ (taken with the sign minus) are shown by the solid and dashed curves in the case of the resonance with the mass $m_{\sigma}=0.6 \mathrm{GeV}$ and the width $\Gamma_{\sigma}=\Gamma_{\sigma \rightarrow \pi \pi}\left(m_{\sigma}^{2}\right)=0.45$ $\mathrm{GeV}$. As may be inferred from this figure, $\operatorname{Re}\left[D_{\sigma}(s)\right]$ can be close to 0 at $s=4 m_{\pi^{+}}^{2}$ due to the correction for the finite width in the case of the large one. Then this results in the threshold cusp in the amplitudes proportional to $\left|1 / D_{\sigma}(s)\right|{ }^{36}$ For reference, in Fig. 29 the real and imaginary parts of the inverse propagator $D_{\sigma}(s)=m_{\sigma}^{2}-s-i m_{\sigma} \Gamma_{\sigma} \sqrt{\left(s-4 m_{\pi^{+}}^{2}\right) /\left(m_{\sigma}^{2}-4 m_{\pi^{+}}^{2}\right)}$

without the correction for the finite width [118] (also taken with the sign minus) are shown by the dotted and dot-dashed curves, respectively, at the same values of $m_{\sigma}$ and $\Gamma_{\sigma}$.

\section{2. $\gamma \gamma \rightarrow \pi^{0} \eta$}

The polarization operators of the $a_{0}$ resonance $\Pi_{a_{0}}^{a b}(s)$ $\left(a b=\pi \eta, K^{+} K^{-}, K^{0} \bar{K}^{0}, \pi \eta^{\prime}\right)$, introduced in Section 4 (see the paragraph with Eqs. (43) and (44)), have the following form: for $s \geq m_{a b}^{(+)} 2\left(m_{a b}^{( \pm)}=m_{b} \pm m_{a}, m_{b} \geq\right.$ $\left.m_{a}\right)$

$$
\begin{array}{r}
\Pi_{a_{0}}^{a b}(s)=\frac{g_{a_{0} \rightarrow a b}^{2}}{16 \pi}\left[\frac{m_{a b}^{(+)} m_{a b}^{(-)}}{\pi s} \ln \frac{m_{a}}{m_{b}}+\rho_{a b}(s) \times\right. \\
\left.\times\left(i-\frac{1}{\pi} \ln \frac{\sqrt{s-m_{a b}^{(-) 2}}+\sqrt{s-m_{a b}^{(+) 2}}}{\sqrt{s-m_{a b}^{(-) 2}}-\sqrt{s-m_{a b}^{(+) 2}}}\right)\right],
\end{array}
$$

where

$$
\rho_{a b}(s)=\sqrt{s-m_{a b}^{(+)} 2} \sqrt{s-m_{a b}^{(-) 2}} / s,
$$

for

36 The references to papers, in which the the finite width corrections and the analytic properties of the propagators of the realistic $f_{0}(980), a_{0}(980)$, and $\sigma(600)$ resonances have been investigated, are pointed out in Section 2. In connection with the $\gamma \gamma \rightarrow \pi^{0} \eta$ and $\gamma \gamma \rightarrow \pi \pi$ reactions these corrections are discussed also in the papers [101, 108]. $m_{a b}^{(-) 2}<s<m_{a b}^{(+) 2}$

$$
\begin{gathered}
\Pi_{a_{0}}^{a b}(s)=\frac{g_{a_{0} \rightarrow a b}^{2}}{16 \pi}\left[\frac{m_{a b}^{(+)} m_{a b}^{(-)} \ln \frac{m_{a}}{m_{b}}}{\pi s}-\right. \\
\left.-\rho_{a b}(s)\left(1-\frac{2}{\pi} \arctan \frac{\sqrt{m_{a b}^{(+) 2}-s}}{\sqrt{s-m_{a b}^{(-) 2}}}\right)\right],
\end{gathered}
$$

where $\rho_{a b}(s)=\sqrt{m_{a b}^{(+) 2}-s} \sqrt{s-m_{a b}^{(-) 2}} / s$, and for $s \leq$ $m_{a b}^{(-)} 2$

$$
\begin{aligned}
& \Pi_{a_{0}}^{a b}(s)=\frac{g_{a_{0} \rightarrow a b}^{2}}{16 \pi}\left[\frac{m_{a b}^{(+)} m_{a b}^{(-)}}{\pi s} \ln \frac{m_{a}}{m_{b}}-\right. \\
& \left.-\rho_{a b}(s) \frac{1}{\pi} \ln \frac{\sqrt{m_{a b}^{(+) 2}-s}+\sqrt{m_{a b}^{(-)^{2}}-s}}{\sqrt{m_{a b}^{(+) 2}-s}-\sqrt{m_{a b}^{(-) 2}-s}}\right] \text {, }
\end{aligned}
$$

where $\rho_{a b}(s)=\sqrt{m_{a b}^{(+) 2}-s} \sqrt{m_{a b}^{(-) 2}-s} / s$.

The triangle loop integral in Eq. (36) is

$$
\widetilde{I}_{\pi \eta}^{V}(s)=\frac{s}{\pi} \int_{\left(m_{\pi}+m_{\eta}\right)^{2}}^{\infty} \frac{\rho_{\pi \eta}\left(s^{\prime}\right) M_{00}^{\mathrm{Born} V}\left(\gamma \gamma \rightarrow \pi^{0} \eta ; s^{\prime}\right)}{s^{\prime}\left(s^{\prime}-s-i \varepsilon\right)} d s^{\prime},
$$

where

$$
\begin{gathered}
M_{00}^{\text {Born } V}\left(\gamma \gamma \rightarrow \pi^{0} \eta ; s\right)= \\
=\frac{1}{2} \int_{-1}^{1} M_{0}^{\text {Born } V}\left(\gamma \gamma \rightarrow \pi^{0} \eta ; s, \theta\right) d \cos \theta,
\end{gathered}
$$

is the $S$ wave Born amplitude, and the amplitude $M_{0}^{\text {Born } V}\left(\gamma \gamma \rightarrow \pi^{0} \eta ; s, \theta\right)$ is defined by Eq. (38). The functions $\widetilde{I}_{\pi^{0} \eta^{\prime}}^{V}(s)$ and $\widetilde{I}_{K \bar{K}}^{K^{*}}(s)$ in Eq. (36) are calculated similarly and the function $\widetilde{I}_{K^{+} K^{-}}^{K^{+}}\left(s ; x_{2}\right)$ is calculated with Eq. (92) in Appendix 8.3.

For the background phase shifts we use the simplest parametrizations, which are suitable in the physical region of the $\gamma \gamma \rightarrow \pi^{0} \eta$ reaction:

$$
\begin{gathered}
e^{i \delta_{a b}^{b g}(s)}=\left[\left(1+i F_{a b}(s)\right) /\left(1-i F_{a b}(s)\right)\right]^{1 / 2} \\
F_{\pi \eta}(s)=\frac{\sqrt{1-m_{\pi \eta}^{(+) 2} / s}\left(c_{0}+c_{1}\left(s-m_{\pi \eta}^{(+) 2}\right)\right)}{1+c_{2}\left(s-m_{\pi \eta}^{(+) 2}\right)^{2}} \\
F_{K \bar{K}}(s)=f_{K \bar{K}} \sqrt{s}\left(\rho_{K^{+} K^{-}}(s)+\rho_{K^{0}} \bar{K}^{0}(s)\right) / 2 \\
F_{\pi \eta^{\prime}}(s)=f_{\pi \eta^{\prime}} \sqrt{s-m_{\pi \eta^{\prime}}^{(+) 2}}
\end{gathered}
$$

The curves in Figs. 25, 26, and 27 correspond to the following model parameters: $\left(m_{a_{0}}, \quad g_{a_{0} \pi \eta}, \quad g_{a_{0} K^{+} K^{-}}, \quad g_{a_{0} \pi \eta^{\prime}}\right)=(0.9845,4.23,3.79$, -2.13) $\mathrm{GeV} ;\left(m_{a_{0}^{\prime}}, g_{a_{0}^{\prime} \pi \eta}, g_{a_{0}^{\prime} K^{+} K^{-}}, g_{a_{0}^{\prime} \pi \eta^{\prime}}\right)=(1.4,3.3$, 
$0.28,2.91) \mathrm{GeV} ; \quad\left(g_{a_{0} \gamma \gamma}, g_{a_{0} \gamma \gamma}\right)=(1.77,-11.5) \times 10^{-3}$ $\mathrm{GeV}^{-1} ; \quad C_{a_{0} a_{0}^{\prime}}=0.06 \mathrm{GeV}^{2}, \quad c_{0}=-0603, \quad c_{1}=-6.48$ $\mathrm{GeV}^{-2}, \quad c_{2}=0.121 \mathrm{GeV}^{-4} ; \quad\left(f_{K \bar{K}}, \quad f_{\pi \eta^{\prime}}\right)=(-0.37$, 0.28) $\quad \mathrm{GeV}^{-1} ; \quad\left(m_{a_{2}}, \quad \Gamma_{a_{2}}^{\text {tot }}\right)=(1.322, \quad 0.116) \quad \mathrm{GeV}$; $\Gamma_{a_{2} \rightarrow \gamma \gamma}^{(0)}=1.053 \mathrm{keV}, r_{a_{2}}=1.9 \mathrm{GeV}^{-1}, \theta_{P}=-24^{\circ}$ (see Ref. [105] for details).

\section{3. $\gamma \gamma \rightarrow K \bar{K}$}

The Born amplitude of the reaction $\gamma \gamma \rightarrow K^{+} K^{-}$, caused by the elementary one kaon exchange, $M_{\lambda}^{\text {Born } K^{+}}(s, \theta)$ and $M_{\lambda J}^{\text {Born } K^{+}}(s)$ result from the corresponding $\gamma \gamma \rightarrow \pi^{+} \pi^{-}$Born amplitudes $M_{\lambda}^{\text {Born } \pi^{+}}(s, \theta)$ and $M_{\lambda J}^{\text {Born } \pi^{+}}(s)$ by the substitution of $m_{K^{+}}$for $m_{\pi^{+}}$and of $\rho_{K^{+}}(s)=\sqrt{1-4 m_{K^{+}}^{2} / s}$ for $\rho_{\pi^{+}}(s)$ in Eqs. (48), (49), and (51)-(53):

$$
\begin{gathered}
M_{0}^{\text {Born } K^{+}}(s, \theta)=\frac{4 m_{K^{+}}^{2}}{s} \frac{8 \pi \alpha}{1-\rho_{K^{+}}^{2}(s) \cos ^{2} \theta}, \\
M_{2}^{\text {Born } K^{+}}(s, \theta)=\frac{8 \pi \alpha \rho_{K^{+}}^{2}(s) \sin ^{2} \theta}{1-\rho_{K^{+}}^{2}(s) \cos ^{2} \theta}, \\
M_{00}^{\text {Born } K^{+}}(s)=4 \pi \alpha \frac{1-\rho_{K^{+}}^{2}(s)}{\rho_{K^{+}}(s)} \ln \frac{1+\rho_{K^{+}}(s)}{1-\rho_{K^{+}}(s)}, \\
M_{02}^{\text {Born } K^{+}}(s)=4 \pi \alpha \frac{1-\rho_{K^{+}}^{2}(s)}{\rho_{K^{+}}^{2}(s)}\left[\frac{3-\rho_{K^{+}}^{2}(s)}{2 \rho_{K^{+}}(s)} \times\right. \\
\left.\times \ln \frac{1+\rho_{K^{+}}(s)}{1-\rho_{K^{+}}(s)}-3\right], \\
M_{22}^{\text {Born } K^{+}}(s)=4 \pi \alpha \sqrt{\frac{3}{2}\left[\frac{\left(1-\rho_{K^{+}}^{2}\right.}{2 \rho_{K^{+}}^{3}(s)}\right)^{2}} \ln \frac{1+\rho_{K^{+}}(s)}{1-\rho_{K^{+}}(s)}- \\
\left.\quad-\frac{1}{\rho_{K^{+}}^{2}(s)}+\frac{5}{3}\right] .
\end{gathered}
$$

The function $\widetilde{I}_{K^{+} K^{-}}^{K^{+}}(s)$ results from $\widetilde{I}_{\pi^{+} \pi^{-}}^{\pi^{+}}(s)$ by the substitution in Eqs. (55) and (56) of $m_{K^{+}}$for $m_{\pi^{+}}$and of $\rho_{K^{+}}(s)$ for $\rho_{\pi^{+}}(s)$, and thus for $0<s<4 m_{K^{+}}^{2}$

$$
\widetilde{I}_{K^{+} K^{-}}^{K^{+}}(s)=8 \alpha\left\{\frac{m_{K^{+}}^{2}}{s}\left[\pi-2 \arctan \left|\rho_{K^{+}}(s)\right|\right]^{2}-1\right\}
$$

and for $s \geq 4 m_{K^{+}}^{2}$

$$
\widetilde{I}_{K^{+} K^{-}}^{K^{+}}(s)=8 \alpha\left\{\frac{m_{K^{+}}^{2}}{s}\left[\pi+i \ln \frac{1+\rho_{K^{+}}(s)}{1-\rho_{K^{+}}(s)}\right]^{2}-1\right\} .
$$

Taking account of the form factor

$$
G_{K^{+}}(t, u)=\frac{1}{s}\left[\frac{m_{K^{+}}^{2}-t}{1-\left(u-m_{K^{+}}^{2}\right) / x_{2}^{2}}+\frac{m_{K^{+}}^{2}-u}{1-\left(t-m_{K^{+}}^{2}\right) / x_{2}^{2}}\right]
$$

(here $t=m_{K^{+}}^{2}-s\left[1-\rho_{K^{+}}(s) \cos \theta\right] / 2$ and $u=m_{K^{+}}^{2}-s[1$ $\left.\left.+\rho_{K^{+}}(s) \cos \theta\right] / 2\right)$, the partial amplitudes $M_{\lambda J}^{\text {Born } K^{+}}(s)$ are replaced by $M_{\lambda J}^{\text {Born } K^{+}}\left(s ; x_{2}\right)$. Substituting $\rho_{K^{+}}(s)$ instead $\rho_{\pi^{+}}(s)$ and $\rho_{K^{+}}\left(s ; x_{2}\right)=\rho_{K^{+}}(s) /\left(1+2 x_{2}^{2} / s\right)$ instead $\rho_{\pi^{+}}\left(s ; x_{1}\right)$ in Eqs. (57)-(60), one gets

$$
\begin{aligned}
M_{0 J}^{\text {Born } K^{+}}(s) & =\frac{1-\rho_{K^{+}}^{2}(s)}{\rho_{K^{+}}(s)} F_{0 J}^{\text {Born } K^{+}}\left(\rho_{K^{+}}(s)\right) \\
M_{2 J}^{\text {Born } K^{+}}(s) & =\rho_{K^{+}}(s) F_{2 J}^{\text {Born } K^{+}}\left(\rho_{K^{+}}(s)\right), \\
M_{0 J}^{\text {Born } K^{+}}\left(s ; x_{2}\right)= & \frac{1-\rho_{K^{+}}^{2}(s)}{\rho_{K^{+}}(s)}\left[F_{0 J}^{\text {Born } K^{+}}\left(\rho_{K^{+}}(s)\right)-\right. \\
& \left.-F_{0 J}^{\text {Born } K^{+}}\left(\rho_{K^{+}}\left(s ; x_{2}\right)\right)\right] \\
M_{2 J}^{\text {Born } K^{+}}\left(s ; x_{2}\right) & =\rho_{K^{+}}(s)\left[F_{2 J}^{\text {Born } K^{+}}\left(\rho_{K^{+}}(s)\right)-\right. \\
- & \left.F_{2 J}^{\text {Born } K^{+}}\left(\rho_{K^{+}}\left(s ; x_{2}\right)\right)\right]
\end{aligned}
$$

Correspondingly, with taking into account the form factor, the function $\widetilde{I}_{K^{+} K^{-}}^{K^{+}}(s)$ is replaced by

$$
\widetilde{I}_{K^{+} K^{-}}^{K^{+}}\left(s ; x_{2}\right)=\frac{s}{\pi} \int_{4 m_{K^{+}}^{2}}^{\infty} \frac{\rho_{K^{+}}\left(s^{\prime}\right) M_{00}^{\mathrm{Born} K^{+}}\left(s^{\prime} ; x_{2}\right)}{s^{\prime}\left(s^{\prime}-s-i \varepsilon\right)} d s^{\prime} .
$$

Note that $0.68 \times\left|\widetilde{I}_{K^{+} K^{-}}^{K^{+}}(s)\right|^{2}$ coincides with $\left|\widetilde{I}_{K^{+} K^{-}}^{K^{+}}\left(s ; x_{2}\right)\right|^{2}$ within an accuracy better than $3 \%$ in the range $0.8 \mathrm{GeV}<\sqrt{s}<1.2 \mathrm{GeV}$ at $x_{2}=1.75 \mathrm{GeV}$.

The $S$ wave amplitudes of the reactions $\gamma \gamma \rightarrow K^{+} K^{-}$ and $\gamma \gamma \rightarrow K^{0} \bar{K}^{0}$, which we used for estimates in the region of the $K \bar{K}$ thresholds, have the form

$$
\begin{gathered}
M_{00}\left(\gamma \gamma \rightarrow K^{+} K^{-} ; s\right)=M_{00}^{\text {Born } K^{+}}\left(s ; x_{2}\right)+ \\
+\widetilde{I}_{\pi^{+} \pi^{-}}^{\pi+}\left(s ; x_{1}\right) T_{\pi^{+} \pi^{-} \rightarrow K^{+} K^{-}}(s)+\widetilde{I}_{K^{+} K^{-}}^{K^{+}}\left(s ; x_{2}\right) \times \\
\times T_{K^{+} K^{-} \rightarrow K^{+} K^{-}}(s)+M_{\mathrm{res} ;+}^{\text {direct }}(s) \\
M_{00}\left(\gamma \gamma \rightarrow K^{0} \bar{K}^{0} ; s\right)= \\
=\widetilde{I}_{\pi^{+} \pi^{-}}^{\pi+}\left(s ; x_{1}\right) T_{\pi^{+} \pi^{-} \rightarrow K^{0} \bar{K}^{0}}(s)+\widetilde{I}_{K^{+} K^{-}}^{K^{+}}\left(s ; x_{2}\right) \times \\
\times T_{K^{+} K^{-} \rightarrow K^{0} \bar{K}^{0}}(s)+M_{\mathrm{res} ;-}^{\text {direct }}(s) .
\end{gathered}
$$

The corresponding cross section are

$$
\sigma_{00}\left(\gamma \gamma \rightarrow K^{+} K^{-}\right)=\frac{\rho_{K^{+}}(s)}{32 \pi s}\left|M_{00}\left(\gamma \gamma \rightarrow K^{+} K^{-} ; s\right)\right|^{2},
$$

$$
\sigma_{00}\left(\gamma \gamma \rightarrow K_{S}^{0} K_{S}^{0}\right)=\frac{\rho_{K^{0}}(s)}{64 \pi s}\left|M_{00}\left(\gamma \gamma \rightarrow K^{0} \bar{K}^{0} ; s\right)\right|^{2}
$$

The amplitudes of the $\pi \pi \rightarrow K \bar{K}$ reactions, $T_{\pi^{+} \pi^{-} \rightarrow K^{+} K^{-}}(s)=T_{\pi^{+} \pi^{-} \rightarrow K^{0} \bar{K}^{0}}(s)=T_{K^{+} K^{-} \rightarrow \pi^{+} \pi^{-}}(s)$, 
are defined by Eqs. (23) and (26). The $K^{+} K^{-} \rightarrow K^{+} K^{-}$ and $K^{+} K^{-} \rightarrow K^{0} \bar{K}^{0}$ reaction amplitudes are given by

$$
\begin{aligned}
& T_{K^{+} K^{-} \rightarrow K^{+} K^{-}}(s)=\left[t_{0}^{0}(s)+t_{0}^{1}(s)\right] / 2, \\
& T_{K^{+} K^{-} \rightarrow K^{0} \bar{K}^{0}}(s)=\left[t_{0}^{0}(s)-t_{0}^{1}(s)\right] / 2,
\end{aligned}
$$

where $t_{0}^{I}(s)$ are the $S$ wave $K \bar{K} \rightarrow K \bar{K}$ reaction amplitudes with isospin $I=0$ and 1 ;

$$
\begin{array}{r}
t_{0}^{0}(s)=\frac{e^{2 i \delta_{B}^{K \bar{K}}(s)}-1}{2 i \rho_{K^{+}}(s)}+e^{2 i \delta_{B}^{K \bar{K}}(s)} T_{\text {res } ; 0}^{K \bar{K}}(s), \\
t_{0}^{1}(s)=\frac{e^{2 i \delta_{K \bar{K}}^{b g}(s)}-1}{2 i \rho_{K^{+}}(s)}+e^{2 i \delta_{K \bar{K}}^{b g}(s)} T_{\text {res } ; 1}^{K \bar{K}}(s),
\end{array}
$$

where $\delta_{B}^{K \bar{K}}(s)$ and $\delta_{K \bar{K}}^{b g}(s)$ are the phases in the channels with $I=0$ and 1 , respectively.

$$
T_{\mathrm{res} ; 0}^{K \bar{K}}(s)=\frac{g_{\sigma K^{+} K^{-}} \bar{\Delta}_{f_{0}}^{0}(s)+g_{f_{0} K^{+} K^{-}} \bar{\Delta}_{\sigma}^{0}(s)}{8 \pi\left[D_{\sigma}(s) D_{f_{0}}(s)-\Pi_{f_{0} \sigma}^{2}(s)\right]},
$$

$$
T_{\mathrm{res} ; 1}^{K \bar{K}}(s)=\frac{g_{a_{0} K^{+} K^{-}} \bar{\Delta}_{a_{0}^{\prime}}^{1}(s)+g_{a_{0}^{\prime} K^{+} K^{-}} \bar{\Delta}_{a_{0}}^{1}(s)}{8 \pi\left[D_{a_{0}}(s) D_{a_{0}^{\prime}}(s)-\Pi_{a_{0} a_{0}^{\prime}}^{2}(s)\right]},
$$

where

$$
\begin{aligned}
& \bar{\Delta}_{f_{0}}^{0}(s)=D_{f_{0}}(s) g_{\sigma K^{+} K^{-}}+\Pi_{f_{0} \sigma}(s) g_{f_{0} K^{+} K^{-}}, \\
& \bar{\Delta}_{\sigma}^{0}(s)=D_{\sigma}(s) g_{f_{0} K^{+} K^{-}}+\Pi_{f_{0} \sigma}(s) g_{\sigma K^{+} K^{-}}, \\
& \bar{\Delta}_{a_{0}^{\prime}}^{1}(s)=D_{a_{0}^{\prime}}(s) g_{a_{0} K^{+} K^{-}}+\Pi_{a_{0} a_{0}^{\prime}}(s) g_{a_{0}^{\prime} K^{+} K^{-}}, \\
& \bar{\Delta}_{a_{0}}^{1}(s)=D_{a_{0}}(s) g_{a_{0}^{\prime} K^{+} K^{-}}+\Pi_{a_{0} a_{0}^{\prime}}(s) g_{a_{0} K^{+} K^{-}} .
\end{aligned}
$$

The amplitudes of the direct resonance transitions into photons are given by

$$
\begin{array}{r}
M_{\mathrm{res} ; \pm}^{\text {direct }}(s)=s e^{i \delta_{B}^{K \bar{K}}(s)} \frac{g_{\sigma \gamma \gamma}^{(0)} \bar{\Delta}_{f_{0}}^{0}(s)+g_{f_{0} \gamma \gamma}^{(0)} \bar{\Delta}_{\sigma}^{0}(s)}{D_{\sigma}(s) D_{f_{0}}(s)-\Pi_{f_{0} \sigma}^{2}(s)} \\
\pm s e^{i \delta_{K \bar{K}}^{b g}(s)} \frac{g_{a_{0} \gamma \gamma}^{(0)} \bar{\Delta}_{a_{0}^{\prime}}^{1}(s)+g_{a_{0}^{\prime} \gamma \gamma}^{(0)} \bar{\Delta}_{a_{0}}^{1}(s)}{D_{a_{0}}(s) D_{a_{0}^{\prime}}(s)-\Pi_{a_{0} a_{0}^{\prime}}^{2}(s)}
\end{array}
$$

[1] Achasov N N, Ivanchenko V N Nucl. Phys. B 315465 (1989)

[2] Achasov N N, Gubin V V Phys. Rev. D 63094007 (2001)

[3] Achasov N N, Gubin V V Yad. Fiz. 651566 (2002) [Achasov N N, Gubin V VPhys. Atom. Nucl. 651528 (2002)]

[4] Achasov N N Nucl. Phys. A 728425 (2003)

[5] Achasov N N Yad. Fiz. 671552 (2004) [Achasov N N Phys. Atom. Nucl. 671529 (2004)]

[6] Achasov N N, Shestakov G N Phys. Rev. D 495779 (1994)

[7] Achasov N N, Shestakov G N Yad. Fiz. 56, No. 9, 206 (1993) [Achasov N N, Shestakov G N Phys. Atom. Nucl 561270 (1993)]

[8] Achasov N N, Shestakov G N Int. J. Mod. Phys. A 9 3669 (1994)

[9] Achasov N N, Shestakov G N Phys. Rev. Lett. 99072001 (2007)

[10] Amsler C et al. (Particle Data Group) Phys. Lett. B 6671 (2008)

[11] Nakamura K et al. (Particle Data Group) J. Phys. G 37075021 (2010)

[12] Rosenfeld A H et al. (Particle Data Group) Rev. Mod. Phys. 37633 (1965)

[13] Rosenfeld A H et al. (Particle Data Group) Rev. Mod. Phys. 391 (1967)

[14] Barash-Schmidt N et al. (Particle Data Group) Rev. Mod. Phys. 41109 (1969)

[15] Gell-Mann M, Levy M Nuovo Cim. 16705 (1960)

[16] Gell-Mann M Physics 1, 63 (1964)

[17] Levy M Nuovo Cim. A 5223 (1967)

[18] Rittenberg A et al. (Particle Data Group) Rev. Mod. Phys. 43 S1 (1971)
[19] Lasinski T A et al. (Particle Data Group) Rev. Mod. Phys. 45 S1 (1973)

[20] Jaffe R L Phys. Rev. D 15 267, 281 (1977)

[21] Sannino F, Schechter J Phys. Rev. D 5296 (1995)

[22] Törnqvist N A Z. Phys. C 68647 (1995)

[23] Ishida S et al. Prog. Theor. Phys. 95745 (1996)

[24] Harada M, Sannino F, Schechter J Phys. Rev. D 5454 (1996)

[25] Ishida S, in Proceedings of the 7th International Conference on Hadron Spectroscopy (Eds S-U Chung, H Willutzki) AIP Conf. Proc. Vol. 432, p. 705 (1998)

[26] Black D et al. Phys. Rev. D 58054012 (1998)

[27] Black D et al. Phys. Rev. D 59074026 (1999)

[28] Ishida M, in Proceedings of the Possible Existence of sigma-Meson and Its Implication to Hadron Physics (Eds S Ishida et al.) (KEK Proceedings 2000-4); hep-ph/0012325

[29] Barnett R M et al. (Particle Data Group) Phys. Rev. D 541 (1996)

[30] Eidelman S et al. (Particle Data Group) Phys. Lett. B 5921 (2004)

[31] Spanier S, Törnqvist N A, Amsler C Phys. Lett. B 667 $594(2008)$

[32] Amsler C et al. (Note on scalar mesons) J. Phys. G 37 $075021(2010)$

[33] Montanet L Rept. Prog. Phys. 46337 (1983)

[34] Achasov N N, Devyanin S A, Shestakov G N Usp. Fiz. Nauk 142361 (1984) [Achasov N N, Devyanin S A, Shestakov G N Sov. Phys. Usp. 27161 (1984)]

[35] Achasov N N, in Proceedings of the Rheinfels Workshop 1990 on Hadron Mass Spectrum (Eds E Klempt, K Peters) Nucl. Phys. B Proc. Suppl. 21189 (1991)

[36] Achasov N N Usp. Fiz. Nauk 1681257 (1998) [Achasov 
N N Physics-Uspekhi 411149 (1998)]

[37] Achasov N N, in Proceedings of the 8th International Conference On Hadron Spectroscopy (Eds W G Li, Y Z Huang, B S Zou) Nucl. Phys. A 675 279c (2000)

[38] Achasov N N Yad. Fiz. 65573 (2002) [Achasov N N Phys. Atom. Nucl. 65546 (2002)]

[39] Achasov N N, in Proceedings of the Ninth International Conference on Hadron Spectroscopy (Eds D Amelin, A M Zaitsev) AIP Conf. Proc. Vol. 619, p. 112 (2002)

[40] Achasov N N, in Proceedings of the International Symposium on Hadron Spectroscopy, Chiral Symmetry and Relativistic Description of Bound Systems (Eds S Ishida et al.) (KEK Proceedings 2003-7) p. 151

[41] Achasov N N, in Proceedings of the KEK Workshop on Hadron Spectroscopy and Chiral Particle Search in J/Psi Decay Data at BES (Eds K Takamatsu et al.) (KEK Proceedings 2003-10) p. 66

[42] Achasov N N, in Proceedings of the 13th International Seminar QUARKS'2004 (Eds D G Levkov, V A Matveev, V A Rubakov) (INR RAS, Moscow, 2004) p. 110

[43] Achasov N N, in Proceedings of the International Bogolyubov Conference "Problems of Theoretical and Mathematical Physics" (Eds V G Kadyshevsky, A N Sissakian) Phys. Part. Nucl. 36, Suppl. 2, 146 (2005)

[44] Achasov N N, in Proceedings of the 14th International Seminar QUARKS'2006 (Eds S V Demidov et al.) (INR RAS, Moscow, 2007) Vol. 1, p. 29

[45] Achasov N N, in Proceedings of the 15th International Seminar QUARKS'2008 (Eds V A Duk, V A Matveev, V A Rubakov) (INR RAS, Moscow, 2010) Vol. 1, p. 3; arXiv:0810.2601

[46] Achasov N N, in Proceedings of the 14th High-Energy Physics International Conference on Quantum ChromoDynamics (Ed. S Narison) Nucl. Phys. B Proc. Suppl. 186283 (2009)

[47] Achasov N N, in Proceedings of the International Bogolyubov Conference "Problems of Theoretical and Mathematical Physics" devoted to the 100th anniversary of N.N.Bogolyubov's birth (RAS, Moscow, JINR, Dubna, August 2009) Phys. Part. Nucl. 41891 (2010); arXiv:1001.3468

[48] Achasov N N, Shestakov G N Usp. Fiz. Nauk 16153 (1991) [Achasov N N, Shestakov G N Sov. Phys. Usp. 34471 (1991)]

[49] Achasov N N, Shestakov G N, in Proceedings of the International Workshop on $e^{+} e^{-}$Collisions from $\phi$ to $\psi$ (Eds G V Fedotovich, S I Redin) (BINP, Novosibirsk, 2000) p. 294

[50] Delbourgo R, Scadron M D Int. J. Mod. Phys. A 13657 (1998)

[51] Godfrey S, Napolitano J Rev. Mod. Phys. 711411 (1999)

[52] Tuan S F, in Proceedings of the Ninth International Conference on Hadron Spectroscopy (Eds D Amelin, A M Zaitsev) AIP Conf. Proc. Vol. 619, p. 495 (2002)

[53] Tuan S F, in Proceedings of the International Symposium on Hadron Spectroscopy, Chiral Symmetry and Relativistic Description of Bound Systems (Eds S. Ishida et al.) (KEK Proceedings 2003-7) p. 319

[54] Close F E, Törnqvist N A J. Phys. G 28 R249 (2002)

[55] Alford M, Jaffe R L, in Proceedings of the High-Energy Physics Workshop on Scalar Mesons: An Interesting Puzzle for QCD (Ed. A H Fariborz) AIP Conf. Proc.
Vol. 688, p. 208 (2003)

[56] Jaffe R L, Wilczek F Phys. Rev. Lett. 91232003 (2003)

[57] Amsler C, Törnquist N A Phys. Rep. 38961 (2004)

[58] Maiani L et al. Phys. Rev. Lett. 93212002 (2004)

[59] Jaffe R L Phys. Rep. 4091 (2005)

[60] Jaffe R L, in Proceedings of the YKIS Seminar on New Frontiers in QCD: Exotic Hadrons and Hadronic Matter (Tokio, Japan) Progr. Theor. Phys. Suppl. 168127 (2007)

[61] Kalashnikova Yu S et al. Eur. Phys. J. A 24437 (2005)

[62] Caprini I, Colangelo G, Leutwyler H Phys. Rev. Lett. 96132001 (2006)

[63] Bugg D V Eur. Phys. J. C 4757 (2006)

[64] Achasov N N, Kiselev A V, Shestakov G N, in Proceedings of the International Workshop on $e^{+} e^{-}$Collisions from $\phi$ to $\psi$ (Eds A Bondar, S Eidelman) Nucl. Phys. B Proc. Suppl. 162, 127 (2006)

[65] Achasov N N, Kiselev A V, Shestakov G N, in Proceedings of the International Workshop on $e^{+} e^{-}$Collisions from $\phi$ to $\psi$ (Eds C Bibi, G Venanzoni) Nucl. Phys. B Proc. Suppl. 181-182, 169 (2008)

[66] Achasov N N, Shestakov G N, in Proceedings of the 6th International Workshop on $e^{+} e^{-}$Collisions from $\phi$ to $\psi$ (Beijing, China, 2009) Chinese Physics C 34807 (2010)

[67] Achasov N N, Shestakov G N, Invited talk at the 16th International Seminar on High Energy Physics QUARKS-2010 (Kolomna, Russia, June 2010)

[68] Fariborz A H, Jora R, Schechter J Phys. Rev. D 76 014011 (2006)

[69] Fariborz A H, Jora R, Schechter J Phys. Rev. D 77 094004 (2008)

[70] Fariborz A H, Jora R, Schechter J Nucl. Phys. B Proc. Suppl. 186298 (2008)

[71] Fariborz A H, Jora R, Schechter J Phys. Rev. D 79 074014 (2009)

[72] Narison S Phys. Rev. D 73114024 (2006)

[73] Narison S, Talk given at the 14th High-Energy Physics International Conference in Quantum Chromodynamics (Montpellier, France, 2008); arXiv:0811.0563

[74] Törnqvist N A Acta. Phys. Polon. B 382831 (2007)

[75] Klempt E, Zaitsev A Phys. Rep. 4541 (2007)

[76] Maiani L, Polosa A D, Riquer V Phys. Lett. B 651129 (2007)

[77] Pennington M R, in Proceedings of the YKIS Seminar on New Frontiers in QCD: Exotic Hadrons and Hadronic Matter (Tokio, Japan) Progr. Theor. Phys. Suppl. 168143 (2007)

[78] Pennington M R, in Proceedings of the 11th International Conference on Meson-Nucleon Physics and the Structure of the Nucleon (Julich, Germany, 2007) p. 106

[79] van Beveren E, Rupp G, in Proceedings of 11th International Conference on Meson-Nucleon Physics and the Structure of the Nucleon (Eds H Machner, S Krewald)(2007) p. 130

[80] Bystritsky Yu M et al. Phys. Rev. D 77054008 (2007)

[81] Leutwyler H, in Proceedings of the Workshop on Scalar Mesons and Related Topics Honoring 70th Birthday of Michael Scadron AIP Conf. Proc. Vol. 1030, p. 46 (2008)

[82] 't Hooft G et al. Phys. Lett. B 662424 (2008)

[83] Ivashin S, Korchin A Eur. Phys. J. C 5489 (2008)

[84] Ebert D, Faustov R N, Galkin V O Eur. Phys. J. C 60 273 (2009)

[85] Achasov N N, Devyanin S A, Shestakov G N Phys. Lett. 108B 134 (1982) 
[86] Achasov N N, Devyanin S A, Shestakov G N Z. Phys. C 1655 (1982)

[87] Budnev V M et al. Phys. Rep. 15181 (1975)

[88] Kolanoski $\mathrm{H}$ "Two-photon physics at $e^{+} e^{-}$storage rings", Springer Tracts in Modern Physics, Vol. 105 (1984)

[89] Mori T et al. (Belle) in Proceedings of the International Simposium on Hadron Spectroscopy, Chiral Symmetry and Relativistic Description of Bound Systems (Eds S Ishida et al.) (KEK Proceedings 2003-7) p. 159

[90] Mori T et al. (Belle) Phys. Rev. D 75 051101(R) (2007)

[91] Mori T et al. (Belle) J. Phys. Soc. Jpn. 76074102 (2007)

[92] Uehara S et al. (Belle) Phys. Rev. D 78052004 (2008)

[93] Uehara S et al. (Belle) Phys. Rev. D 80032001 (2009)

[94] Marsiske H et al. (Crystal Ball) Phys. Rev. D 413324 (1990)

[95] Boyer J et al. (MARK II) Phys. Rev. D 421350 (1990)

[96] Oest T et al. (JADE) Z. Phys. C 47343 (1990)

[97] Behrend H J et al. (CELLO) Z. Phys. C 56381 (1992)

[98] Bienlein $\mathrm{J} \mathrm{K}$, in Proceedings of the 9th International Workshop on Photon-Photon Collisions (Eds D Caldwell, H P Paar) (Singapore: World Scientific, 1992) p. 241

[99] Barate R et al. (ALEPH) Phys. Lett B 472189 (2000)

[100] Braccini S, in Proceedings of the Meson 2000 Workshop (Eds L Jarczyk at al.) Acta Phys. Polon. B 312143 (2000)

[101] Achasov N N, Shestakov G N Phys. Rev. D 72013006 (2005)

[102] Achasov N N, Shestakov G N Phys. Rev. D 77074020 (2008)

[103] Achasov N N, Shestakov G N Pis'ma Zh. Eksp. Teor. Fiz. 88345 (2008) [Achasov N N, Shestakov G N JETP Lett. 88295 (2008)]

[104] Achasov N N, Shestakov G N Pis'ma Zh. Eksp. Teor. Fiz. 90355 (2009) [Achasov N N, Shestakov G N JETP Lett. 90313 (2009)]

[105] Achasov N N, Shestakov G N Phys. Rev. D 81094029 (2010)

[106] Achasov N N, Shestakov G N Pis'ma Zh. Eksp. Teor. Fiz. 923 (2010) [Achasov N N, Shestakov G N JETP Lett. 921 (2010)]

[107] Achasov N N, Devyanin S A, Shestakov G N Z. Phys. C 27, 99 (1985)

[108] Achasov N N, Shestakov G N Z. Phys. C 41309 (1988)

[109] Achasov N N, Shestakov G N Yad. Fiz. 552999 (1992) [Achasov N N, Shestakov G N Sov. J. Nucl. Phys. 55, $1677(1992)]$

[110] Achasov N N, Shestakov G N Mod. Phys. Lett. A 91351 (1994)

[111] Flatte S M et al. Phys. Lett. B 38232 (1972)

[112] Protopopescu S D et al. Phys. Rev. D 71279 (1973)

[113] Hyams B et al. Nucl. Phys. B 64134 (1973)

[114] Grayer G et al. Nucl. Phys. B 75189 (1974)

[115] Hyams B et al. Nucl. Phys. B 100205 (1975)

[116] Gay J et al. Phys. Lett. B 63220 (1976)

[117] Morgan D Phys. Lett. B 5171 (1974)

[118] Flatte S M Phys. Lett. B 63 224, 228 (1976)

[119] Martin A D, Ozmutlu E N, Squires E J Nucl. Phys. B 121514 (1977)

[120] Petersen J L "The $\pi \pi$ Interaction", Yellow CERN Preprint 77-04 (Geneva: CERN, 1977)

[121] Estabrooks P Phys. Rev. D 192678 (1979)

[122] Achasov N N, Devyanin S A, Shestakov G N Phys. Lett.
B 88367 (1979)

[123] Achasov N N, Devyanin S A, Shestakov G N Yad. Fiz. 331337 (1981) [Achasov N N, Devyanin S A, Shestakov G N Sov. J. Nucl. Phys. 33715 1981]

[124] Achasov N N, Shestakov G N Phys. Rev. Lett. 92182001 (2004)

[125] Achasov N N, Shestakov G N Phys. Rev. D 70074015 (2004)

[126] Wu J-J, Zhao Q, Zou B S Phys. Rev. D 75114012 (2007)

[127] Wu J-J, Zou B S Phys. Rev. D 78074017 (2008)

[128] Dorofeev V et al., in Proceedings of the 12th International Conference on Hadron Spectroscopy (Frascati Physics Series) Vol. XLVI (2007); arXiv:0712.2512

[129] Dorofeev V et al. Eur. Phys. J. A 38149 (2008)

[130] Nikolaenko V et al. Int. J. Mod. Phys. A 24295 (2009)

[131] Harris F, arXiv:1008.3569

[132] Achasov N N, Devyanin S A, Shestakov G N Yad. Fiz. 321098 (1980) [Achasov N N, Devyanin S A, Shestakov G N Sov. J. Nucl. Phys. 32566 (1980)]

[133] Achasov N N, Devyanin S A, Shestakov G N Phys. Lett. $B 96168$ (1980)

[134] Achasov N N, Devyanin S A, Shestakov G N Phys. Lett. $B 102196$ (1981)

[135] Achasov N N, Devyanin S A, Shestakov G N "On fourquark nature of scalar $S^{*}(980)$ and $\delta(980)$ resonances" Preprint TP-121 (Novosibirsk: Institute for Mathematics, 1981)

[136] Achasov N N, Devyanin S A, Shestakov G N Z. Phys. $C 2253$ (1984)

[137] Achasov M N et al. (SND) Phys. Lett. B 438441 (1998)

[138] Achasov M N et al. (SND) Phys. Lett. B 440442 (1998)

[139] Achasov M N et al. (SND) Phys. Lett. B 47953 (2000)

[140] Achasov M N et al. (SND) Phys. Lett. B 485349 (2000)

[141] Akhmetshin R R et al. (CMD-2) Phys. Lett. B 462371 (1999)

[142] Akhmetshin R R et al. (CMD-2) Phys. Lett. B 462380 (1999)

[143] Aloisio A et al. (KLOE) Phys. Lett. B 536209 (2002)

[144] Aloisio A et al. (KLOE) Phys. Lett. B 53721 (2002)

[145] Dubrovin M, in High-Energy Physics Workshop on Scalar Mesons: An Interesting Puzzle for QCD (Ed A H Fariborz) AIP Conf. Proc. Vol. 688, p. 231 (2004)

[146] Ambrosino F et al. (KLOE) Phys. Lett. B 634148 (2006)

[147] Ambrosino F et al. (KLOE) Eur. Phys. J. C 49473 (2007)

[148] Ambrosino F et al. (KLOE), contributed to 23rd International Symposium on Lepton-Photon Interactions at High Energy (Daegu, Korea, 2007); arXiv: 0707.4609

[149] Bonvicini G et al. (CLEO) Phys. Rev. D 76012001 (2007)

[150] Cavoto G, in Proceedings of the 5th Flavor Physics and CP Violation Conference (Bled, Slovenia, 2007) p. 22; arXiv:0707.1242

[151] Bossi F et al. Riv. Nuovo Cim. 031531 (2008); arXiv:0811.1929

[152] Achasov N N, Kiselev A V Phys. Rev. D 70 111901(R) (2004)

[153] Bramon A, Grau A, Pancheri G Phys. Lett. B 28997 (1992)

[154] Close F E, Isgur N, Kumano S Nucl. Phys. B 389513 (1993)

[155] Lucio J L, Napsuciale M Phys. Lett. B 331418 (1994)

[156] Achasov N N, in The Second DA NE Physics Hand- 
book(Eds L Maiani, G Pancheri, N Paver) Vol. II, p. 671 (1995)

[157] Achasov N N, Gubin V V, Solodov E P Phys. Rev. D 552672 (1997)

[158] Achasov N N, Gubin V V, Solodov E P Yad. Fiz. 60 1279 (1997) [Achasov N N, Gubin V V, Solodov E P Phys. Atom. Nucl. 601152 (1997)]

[159] Achasov N N, Gubin V V, Shevchenko V I Phys. Rev. D 56203 (1997)

[160] Achasov N N, Gubin V V, Shevchenko V I Int. J. Mod. Phys. A 125019 (1997)

[161] Achasov N N, Gubin V V, Shevchenko V I Yad. Fiz. 60 89 (1997) [Achasov N N, Gubin V V, Shevchenko V I Phys. Atom. Nucl. 6089 (1997)]

[162] Achasov N N, Gubin V V Phys. Rev. D 564084 (1997)

[163] Achasov N N, Gubin V V Yad. Fiz. 61274 (1998) [Achasov N N, Gubin V V Phys. Atom. Nucl. 61224 (1998)]

[164] Achasov N N, Gubin V V Phys. Rev. D 571987 (1998)

[165] Achasov N N, Gubin V V Yad. Fiz. 611473 (1998) [Achasov N N, Gubin V V Phys. Atom. Nucl. 611367 (1998)]

[166] Ambrosino F et al. (KLOE) Phys. Lett. B 67910 (2009)

[167] Ambrosino F et al. (KLOE) Phys. Lett. B 6815 (2009)

[168] Bini, C. (KLOE), 2008, Talk given at 34th International Conference on High Energy Physics (Philadelphia, Pennsylvania); arXiv:0809.5004

[169] Achasov N N, Kiselev A V Phys. Rev. D 68014006 (2003)

[170] Achasov N N, Kiselev A V Yad. Fiz. 67653 (2004) [Achasov N N, Kiselev A V Phys. Atom. Nucl. 67633 (2004)]

[171] Achasov N N, Kiselev A V Phys. Rev. D 73054029 (2006)

[172] Achasov N N, Kiselev A V Yad. Fiz. 702005 (2007) [Achasov N N, Kiselev A V Phys. At. Nucl. 701956 (2007)]

[173] Di Micco B, in Proceedings of the International Workshop on $e^{+} e^{-}$Collisions from $\phi$ to $\psi$ (Eds C Bibi, G Venanzoni) Nucl. Phys. B Proc. Suppl. 181-182 215 (2008)

[174] Shekhovtsova O, Venanzoni G, Pancheri G Comput. Phys. Commun. 1801206 (2009).

[175] Amelino-Camelia et al. Eur. Phys. J. C 68619 (2010); arXiv:1003.3868.

[176] Field J H, in Proceedings of the 4th International Colloquium on Photon-Photon Interactions (Singapore: World Scientific, 1981) p. 447

[177] Hilger E, in Proceedings of the 4th International Colloquium on Photon-Photon Interactions (Singapore: World Scientific, 1981) p. 149

[178] Wedemeyer R J, in Proceedings of the 10th International Symposium on Lepton and Photon Interactions at High Energies (Ed W Pfeil) (Bonn Univ.: Phys. Inst., 1981) p. 410

[179] Edwards C et al. (Crystal Ball) Phys. Lett. B 11082 (1982)

[180] Olsson J E, in Proceedings of the 5th International Workshop on Photon-Photon Interactions (Ed C Berger) (Berlin: Springer-Verlag, 1983) Lecture Notes in Physics, Vol. 191, p. 45

[181] Mennessier G Z. Phys. C 16241 (1983)

[182] Kolanoski H, 1985, in Proceedings of the 12th International Symposium on Lepton and Photon Interactions at
High Energies (Eds M Konuma, K Takahashi) (Kyoto Univ.: Research Inst. Fund. Phys., 1986) p. 90

[183] Kolanoski H, Zerwas P, in High Energy ElectronPositron Physics (Eds A Ali, P Söding) (Singapore: World Scientific, 1988) p. 695

[184] Kolanoski H, in Proceedings of the 9th European Symposium on Antiproton-Proton Interactions and Fundamental Symmetries (Eds K Kleinknecht, E Klempt) Nucl. Phys. B Proc. Suppl. 841 (1989)

[185] Cordier A, Proceedings of the 6th International Workshop on Photon-Photon Collisions (Ed R L Lander) (Singapore: World Scientific, 1985) p. 122

[186] Erne F C, Proceedings of the 6th International Workshop on Photon-Photon Collisions (Ed R L Lander) (Singapore: World Scientific, 1985) p. 151

[187] Barnes T Phys. Lett. B 165434 (1985)

[188] Kaloshin A E, Serebryakov V V Z. Phys. C 32279 (1986)

[189] Antreasyan D et al. Phys. Rev. D 331847 (1986)

[190] Johnson R P "Measurements of charged two-particle exclusive states in photon-photon interactions", Ph.D. thesis, (Stanford University, SLAC-Report-294, 1986)

[191] Poppe M Intern. J. Mod. Phys. A 1545 (1986)

[192] Berger Ch, Wagner W Phys. Rep. C 1461 (1987)

[193] Morgan D, Pennington M R Phys. Lett. B 192207 (1987)

[194] Morgan D, Pennington M R Z. Phys. C 37431 (1988)

[195] Chanowitz M S, in Proceedings of the 8th International Workshop on Photon-Photon Collisions (Ed U Karshon) (Singapore: World Scientific, 1988) p. 205

[196] Hikasa K et al. (Particle Data Group) Phys. Rev. D 45 S1 (1992)

[197] Morgan D, Pennington M R, Whalley M R "A compilation of the data on two-photon reactions leading to hadron final states", J. Phys. G 20, Suppl. 8A, A1 (1994)

[198] Barnes T, in Proceedings of the 9th International Workshop on Photon-Photon Collisions (Eds D O Caldwell, H P Paar) (Singapore: World Scientific, 1992) p. 263, p. 275

[199] Kolanoski H, in Proceedings of the 4th International Conference on Hadron Spectroscopy (Eds S Oneda, D C Peaslee) (Singapore: World Scientific, 1992) p. 377

[200] Karch K-H, in Proceedings of the 26th Rencontre de Moriond: High Energy Hadronic Interactions (Ed J Tran Thanh Van) (France: Gif-sur-Yvette, Editions Frontieres, 1991), p. 423

[201] Cahn R N, in Proceedings of the 14th International Symposium on Lepton and Photon at High Energies (Ed M Riordan) (Singapore: World Scientific, 1990) p. 60

[202] Feindt M, Harjes J, in Proceedings of the Rheinfeld 1990 Workshop on the Hadron Mass Specrum (Eds E Klempt, K Peters) Nucl. Phys. B Proc. Suppl. 2161 (1991)

[203] Berger S B, Feld B T Phys. Rev. D 83875 (1973)

[204] Barbieri R, Gatto R, Kögerler R Phys. Lett. 60B 183 (1976)

[205] Jackson J D, in Proceedings of the SLAC Summer Institute on Particle Physics: Weak Interactions at High Energy and the Production of New Particles (Ed M C Zipf) SLAC Rep. No. 198, p. 147 (1976)

[206] Budnev V M, Kaloshin A E Phys. Lett. B 86351 (1979)

[207] Bergström L, Hulth G, Snellman H Z. Phys. C 16263 (1983)

[208] Morgan D, Pennington M R Z. Phys. C 48623 (1990) 
[209] Li Z P, Close F E, Barnes T Phys. Rev. D 432161 (1991)

[210] Münz C R Nucl. Phys. A 609364 (1996)

[211] Pennington M R Mod. Phys. Lett. A 221439 (2007)

[212] Weinstein J, Isgur N Phys. Rev. Lett. 48652 (1982)

[213] Weinstein J, Isgur N Phys. Rev. D 412236 (1990)

[214] Achasov N N, Kiselev A V Phys. Rev. D 76077501 (2007)

[215] Achasov N N, Kiselev A V Phys. Rev. D 78058502 (2008)

[216] Dzierba A R, in Proceedings of the Second Workshop on Physics and Detectors for DAФNE'95 (Eds R Baldini et al.) (Frascati Physics Series, 1996) Vol. 4, p. 99

[217] Alde D et al. Yad. Fiz. 62462 (1999) [Alde D et al. Phys. At. Nucl. 62421 (1999)]

[218] Alde D et al. Z. Phys. C 66375 (1995)

[219] Alde D et al. Eur. Phys. J. A 3361 (1998)

[220] Gunter J et al. Phys. Rev. D 64072003 (2001)

[221] Branz T, Gutsche T, Lyubovitskij V E Eur. Phys. J. A 37303 (2007)

[222] Boglione M, Pennington M R Eur. Phys. J. C 911 (1999)

[223] Pennington M R, in Proceedings of the International Conference on the Structure and Interactions of the Photon (Ed S Soldner-Rembold) Nucl. Phys. B Proc. Suppl. 82291 (2000)

[224] Groom D E et al. (Particle Data Group) Eur. Phys. J. $C 151$ (2000)

[225] Yao W M et al. (Particle Data Group) J. Phys. G 331 (2006)

[226] Abe K et al. (Belle) arXiv:0711.1926

[227] Nakazawa N, in Proceedings of the International Workshop on $e^{+} e^{-}$Collisions from $\phi$ to $\psi$ (Ed C Bibi, G. Venanzoni) Nucl. Phys. B Proc. Suppl. 181-182, 233 (2008).

[228] Adachi I et al. (Belle) arXiv:0810.0334

[229] Low F E Phys. Rev. 961428 (1954)

[230] Gell-Mann M, Goldberger M L Phys. Rev. 961433 (1954)

[231] Abarbanel H D I, Goldberger M L Phys. Rev. 1651594 (1968)

[232] Lyth D H J. Phys. G 1039 (1984)

[233] Lyth D H J. Phys. G 11459 (1985)

[234] Bijnens J, Cornet F Nucl. Phys. B 296557 (1988)

[235] Donoghue J F, Holstein B R, Lin Y C Phys. Rev. D 37 2423 (1988)

[236] Donoghue J F, Holstein B R Phys. Rev. D 48137 (1993)

[237] Morgan D, Pennington M R Phys. Lett. B 272134 (1991)

[238] Oller J A, Oset E., in Proceedings of the 7th International Conference on Hadron Spectroscopy (Eds S-U Chung, H Willutzki) AIP Conf. Proc. Vol. 432, p. 413 (1998)

[239] Pennington M R Phys. Rev. Lett. 97011601 (2006)

[240] Krammer M, Krasemann H Phys. Lett. B 7358 (1978)

[241] Krammer M Phys. Lett. B 74361 (1978)

[242] Krasemann H, Vermaseren J A M Nucl Phys. B 184 269 (1981)

[243] Gersten A Nucl. Phys. B 12537 (1969)

[244] Barrelet E Nuovo Cim. A 8331 (1972)

[245] Sadovsky S A Yad. Fiz. 62562 (1999) [Sadovsky S A Phys. Atom. Nucl. 62519 (1999)]

[246] Pennington M R, in Proceedings of the International Workshop on $e^{+} e^{-}$Collisions from $\phi$ to $\psi$ (Eds C Bibi G Venanzoni) Nucl. Phys. B Proc. Suppl. 181-182 251
(2008)

[247] Pennington M R et al. Eur. Phys. J. C 561 (2008)

[248] Mennessier G et al. arXiv:0707.4511

[249] Mennessier G, Narison S, Ochs W Phys. Lett. B 665 205 (2008)

[250] Mennessier G, Narison S, Ochs W, in Proceedings of the International Workshop on $e^{+} e^{-}$Collisions from $\phi$ to $\psi$ (Eds C Bibi, Venanzoni G) Nucl. Phys. B Proc. Suppl. 181-182 238 (2008)

[251] Mennessier G, Talk given at the 14th High-Energy Physics International Conference in Quantum Chromodynamics (Montpellier, France, 2008), arXiv: 0811.1589

[252] Oller J A, Roca L, Schat C Phys. Lett. B 659201 (2008)

[253] Oller J A, Roca L Eur. Phys. J. A 3715 (2008)

[254] van Beveren E et al. Phys. Rev. D 79098501 (2009)

[255] Kalinovsky Yu L, Volkov M K, arXiv:0809.1795

[256] Mao Y at al. Phys. Rev. D 79116008 (2009)

[257] Mennessier G, Narison S, Wang X-G, arXiv:1009.2773

[258] Garsia-Martin R, Moussallam B Eur. Phys. J 70155 (2010)

[259] Adler S L Phys. Rev. 1772426 (1969)

[260] Bell J S, Jackiw L Nuovo Cim. A 6047 (1969)

[261] Bardeen W A, Fritzsch H, Gell-Mann M, in Proceedings of the Meeting on Scale and Conformal Symmetry in Hadron Physics (Ed R Gatto) (Wiley, 1973); hep-ph/0211388

[262] Leutwyler H Nucl. Phys. B Proc. Suppl. 64223 (1998)

[263] Ioffe B L, Oganesian A G Phys. Lett. B 647389 (2007)

[264] Bernstein A M, arXiv:0707.4250

[265] Feldmann T Int. J. Mod. Phys. A 15159 (2000)

[266] Babcock J, Rosner J L Phys. Rev. D 141286 (1976)

[267] Rosner J L Phys. Rev. D 231127 (1981)

[268] Berger $\mathrm{Ch}$, in Proceedings of the International Workshop on gamma gamma Collisions (Eds G Cochard, P Kessler) (Berlin: Springer Verlag, 1980) Lecture Notes in Physics, Vol. 134, p. 82

[269] Albrecht H et al. (ARGUS) Z. Phys. C 48183 (1990)

[270] Li D-M, Yu H, Shen Q-X J. Phys. G 27807 (2001)

[271] Durusoy N B et al. Phys. Lett. B 45517 (1973)

[272] Hoogland W et al. Nucl. Phys. B 126109 (1977)

[273] Watson K M Phys. Rev. 881163 (1952)

[274] Achasov N N, Shestakov G N Phys. Rev. D 67114018 (2003)

[275] Achasov N N, Shestakov G N Phys. Rev. D 58054011 (1998)

[276] Osborn H Nucl. Phys. B 15501 (1970)

[277] Petersen J L Phys. Rep. 2155 (1971)

[278] Bernard V, Kaiser N, Meissner U-G Phys. Rev. D 44 3698 (1991)

[279] Black D, Fariborz A H, Schechter J Phys.Rev. D 61 $074030(2000)$

[280] Achasov N N, Shestakov G N Phys. Rev. D 533559 (1996)

[281] Ochs W, in Proceedings of the XIII International Conference on Hadron Spectroscopy (Tallahassee, Florida, 2009) AIP Conf. Proc. Vol. 1257, p. 252 (2010)

[282] Kalashnikova Yu et al. Phys. Rev. C 7345203 (2006)

[283] Hanhart C et al. Phys. Rev. D 75074015 (2007)

[284] Czerwinski E, arXiv:1009.0113.

[285] Uehara S et al., arXiv:1007.3779

[286] Althoff M et al. (TASSO) Z. Phys. C 29189 (1985)

[287] Althoff M et al. (TASSO) Phys. Lett. B 121216 (1983)

[288] Berger Ch et al. (PLUTO) Z. Phys. C 37329 (1988)

[289] Behrend H J et al. (CELLO) Z. Phys. C 4391 (1989) 
[290] Feindt M, Harjes J, in Proceedings of the Rheinfeld 1990 Workshop on the Hadron Mass Specrum (Eds E Klempt, K Peters) Nucl. Phys. B Proc. Suppl. 2161 (1991)

[291] Acciarri M et al. (L3) Phys. Lett. B 501173 (2001)

[292] Abe K et al. (Belle) Eur. Phys. J. C 32323 (2004)

[293] Faiman D, Lipkin H J, Rubinstein H R Phys. Lett. B
59269 (1975)

[294] Achard P et al. Phys. Lett. B 56811 (2003)

[295] Achard P et al. Phys. Lett. B 59726 (2004)

[296] Achard P et al. Phys. Lett. B 60448 (2004)

[297] Achard P et al. Phys. Lett. B 61519 (2005) 